\title{
WestVirginiaUniversity
}

THE RESEARCH REPOSITORY @ WVU

Graduate Theses, Dissertations, and Problem Reports

2021

\section{Weed Recognition in Agriculture: A Mask R-CNN Approach}

Sruthi Keerthi Valicharla

West Virginia University, sv0031@mix.wvu.edu

Follow this and additional works at: https://researchrepository.wvu.edu/etd

Part of the Other Electrical and Computer Engineering Commons

\section{Recommended Citation}

Valicharla, Sruthi Keerthi, "Weed Recognition in Agriculture: A Mask R-CNN Approach" (2021). Graduate Theses, Dissertations, and Problem Reports. 8102.

https://researchrepository.wvu.edu/etd/8102

This Thesis is protected by copyright and/or related rights. It has been brought to you by the The Research Repository @WVU with permission from the rights-holder(s). You are free to use this Thesis in any way that is permitted by the copyright and related rights legislation that applies to your use. For other uses you must obtain permission from the rights-holder(s) directly, unless additional rights are indicated by a Creative Commons license in the record and/ or on the work itself. This Thesis has been accepted for inclusion in WVU Graduate Theses, Dissertations, and Problem Reports collection by an authorized administrator of The Research Repository @ WVU. For more information, please contact researchrepository@mail.wvu.edu. 
Graduate Theses, Dissertations, and Problem Reports

2021

Weed Recognition in Agriculture: A Mask R-CNN Approach

Sruthi Keerthi Valicharla

Follow this and additional works at: https://researchrepository.wvu.edu/etd

Part of the Other Electrical and Computer Engineering Commons 


\title{
Weed Recognition in Agriculture: A Mask R-CNN Approach
}

\author{
Sruthi Keerthi Valicharla \\ Thesis submitted to the \\ Benjamin M. Statler College of Engineering and Mineral Resources \\ at West Virginia University \\ in partial fulfillment of the \\ requirements for the degree of \\ Master of Science \\ in \\ Electrical Engineering
}

Xin Li, Ph.D., Chair

Roy S. Nutter, Ph.D.

Katerina Goseva- Popstojanova, Ph.D.

Lane Department of Computer Science and Electrical Engineering

Morgantown, West Virginia 2021

Keywords: Mask R-CNN, Mile-A-Minute, Localized Style Transfer, UAVs, Data Augmentation, Detectron2

Copyright 2021 Sruthi Keerthi Valicharla 


\section{Abstract \\ Weed Recognition in Agriculture: A Mask R-CNN Approach \\ Sruthi Keerthi Valicharla}

Recent interdisciplinary collaboration on deep learning has led to a growing interest in its application in the agriculture domain. Weed control and management are some of the crucial tasks in agriculture to maintain high crop productivity. The inception phase of weed control and management is to successfully recognize the weed plants, followed by providing a suitable management plan. Due to the complexities in agriculture images, such as similar colour and texture, we need to incorporate a deep neural network that uses pixel-wise grouping for identifying the plant species. In this thesis, we analysed the performance of one of the most popular deep neural networks aimed to solve the instance segmentation (pixel-wise analysis) problems: Mask R-CNN, for weed plant recognition (detection and classification) using field images and aerial images. We have used Mask R-CNN to recognize the crop plants and weed plants using the Crop/Weed Field Image Dataset (CWFID) for the field image study. However, the CWFID's limitations are that it identifies all weed plants as a single class and all of the crop plants are from a single organic carrot field. We have created a synthetic dataset with 80 weed plant species to tackle this problem and tested it with Mask R-CNN to expand our study.

Throughout this thesis, we predominantly focused on detecting one specific invasive weed type called Persicaria Perfoliata or Mile-A-Minute (MAM) for our aerial image study. In general, supervised model outcomes are slow to aerial images, primarily due to large image size and scarcity of well-annotated datasets, making it relatively harder to recognize the species from higher altitudes. We propose a three-level (leaves, trees, forest) hierarchy to recognize the species using Unmanned Aerial Vehicles(UAVs) to address this issue. To create a dataset that resembles weed clusters similar to MAM, we have used a localized style transfer technique to transfer the style from the available MAM images to a portion of the aerial images' content using VGG-19 architecture. We have also generated another dataset at a relatively low altitude and tested it with Mask R-CNN and reached $\sim 92 \%$ AP50 using these low-altitude resized images. 


\section{Acknowledgements}

First and foremost, I would like to express my deep appreciation and indebtedness to my committee chair and research advisor, Dr. Xin Li, for his endless support and invaluable advice throughout my MS journey. His expertise in various domains provided me an opportunity to work with the agriculture department. I would also like to extend my gratitude to Dr. Yong-Lak Park for his constant feedback and resources. Without their knowledgeable advice and timely suggestions, this thesis would not have been possible.

I would also like to thank my committee members Dr. Roy S. Nutter and Dr. Katerina GosevaPopstojanova, for their support, time, and encouragement. I'm forever grateful to Dr. Brian M. Powell for providing me an opportunity to work as a GTA for CS 101.

I'm much obliged to my late grandma Vijayalakshmi Velavarthipati for her constant motivation to pursue my higher education. I'm thankful to my cousin Sandhya Mynampati for her continuous love and support. Immense thanks to my friend Anusha Kandula for all the countless favours throughout my life in the States. Special thanks to Dr. Satish GVS.

Finally, I owe my gratitude to my parents Dr. Anuradha Devi Velavarthipati, Sreenivasa Rao V.V, and my brother Surya Praneeth V. for their unconditional love and encouragement. 


\section{Table of Contents}

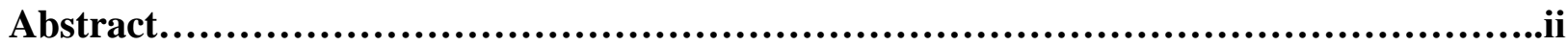

Acknowledgments..........................................................................ii

List of Figures................................................................................ii

List of Tables............................................................................................

Chapter 1: ................................................................................................................................... 1

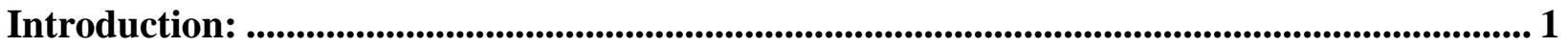

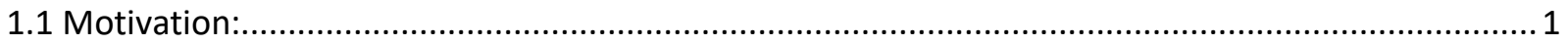

1.1.1 Classification vs. Localization vs. Detection: ......................................................................

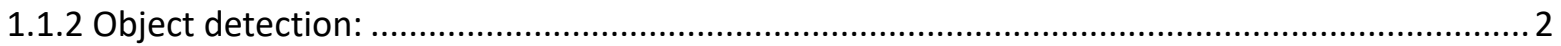

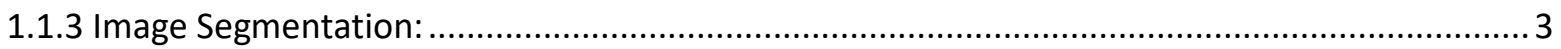

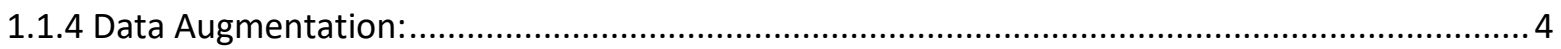

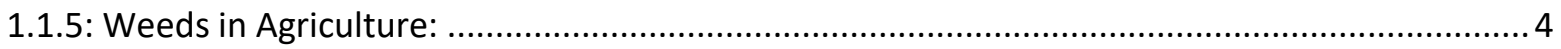

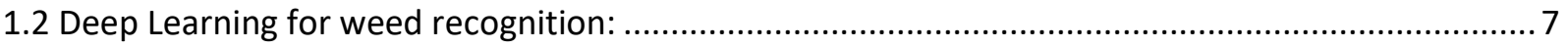

1.3 Problem Statement:

1.3.1 Ground level weed plants recognition using Mask R-CNN: ............................................... 8

1.3.2 Synthetic Dataset creation using Neural Style Transfer and Geometrical transformations: ...... 9

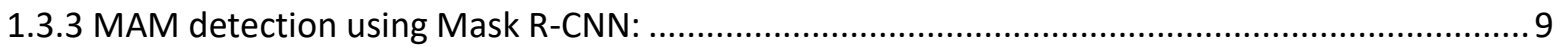

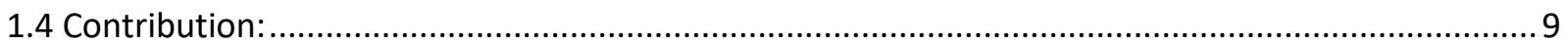

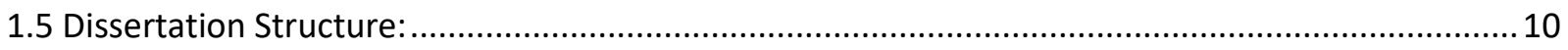

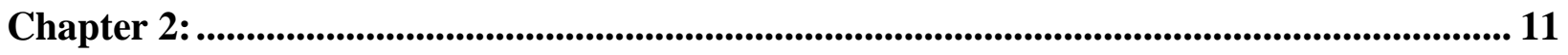

Literature Survey: ................................................................................................................................... 11

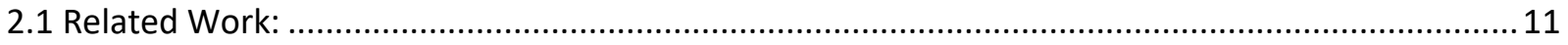

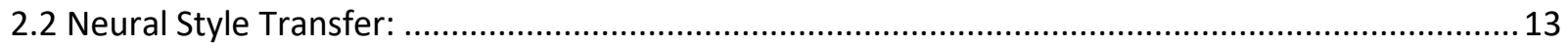

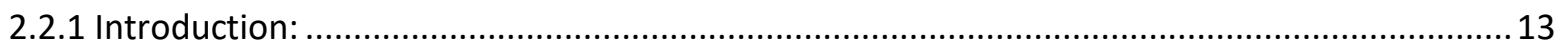

2.2.2 Style transfer vs. localized style transfer: ..........................................................................

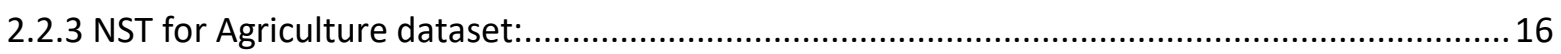

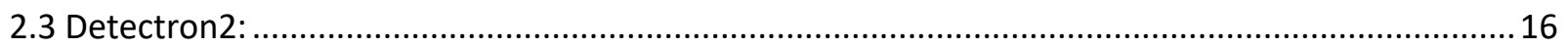

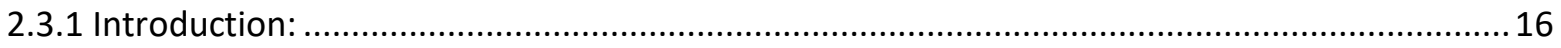

2.3.2 Data Loader, Models, and Data Augmentation: ...................................................................18 


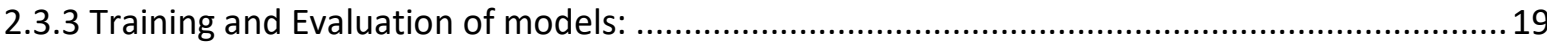

Chapter 3: .......................................................................................................................................... 21

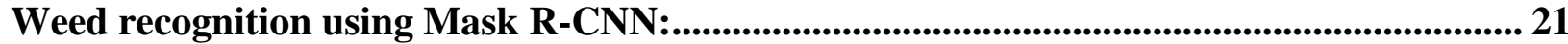

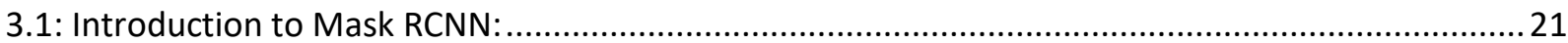

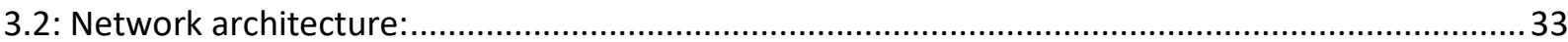

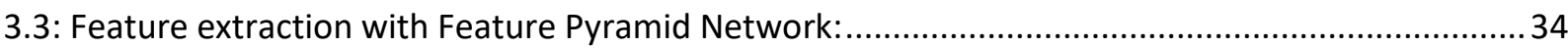

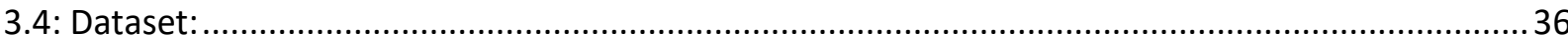

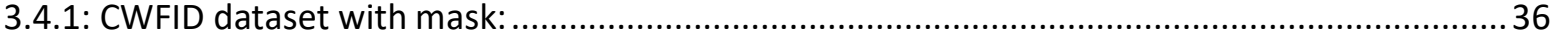

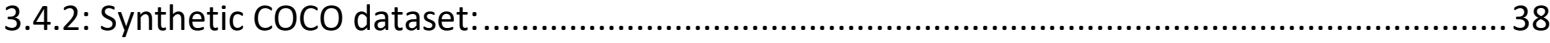

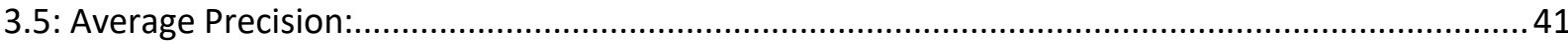

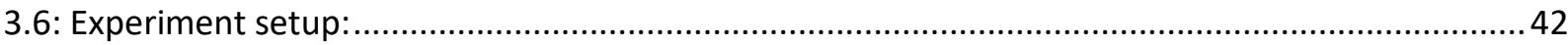

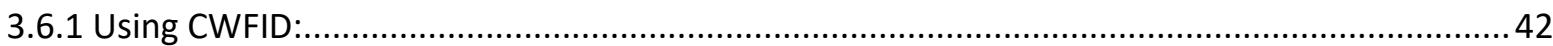

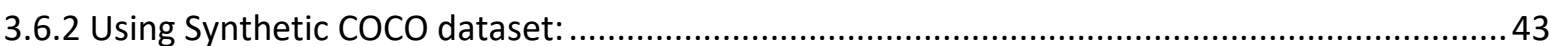

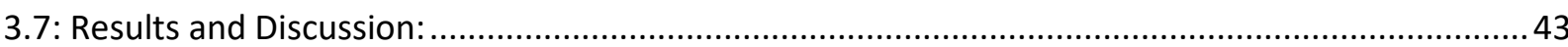

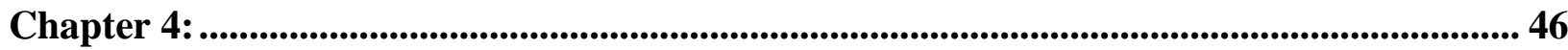

Data Augmentation for Aerial Images:........................................................................................ 46

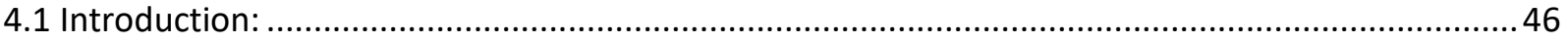

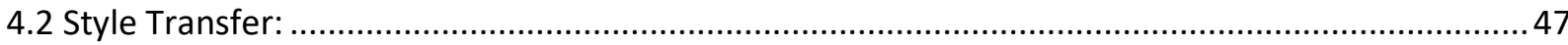

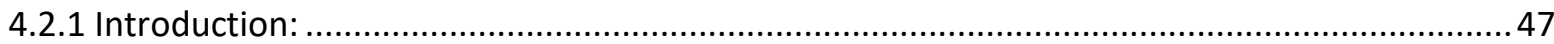

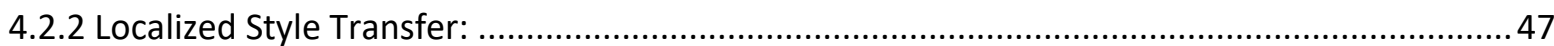

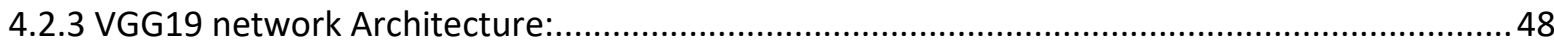

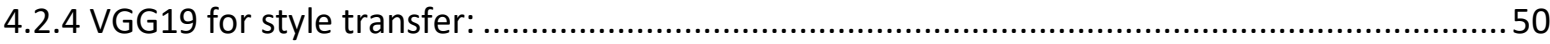

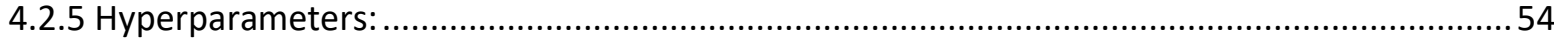

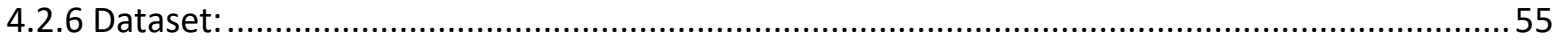

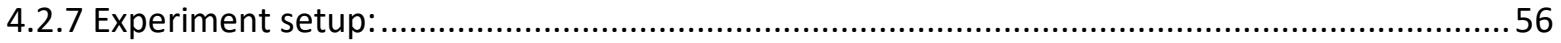

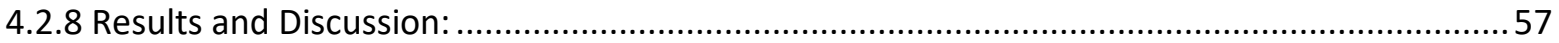

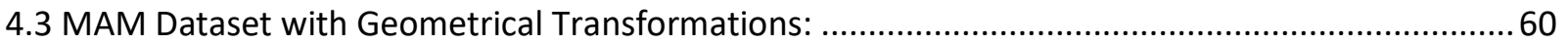

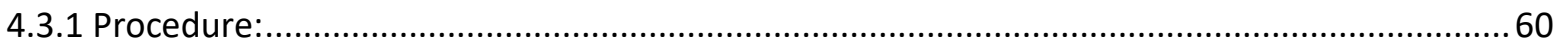

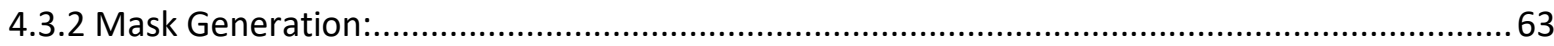

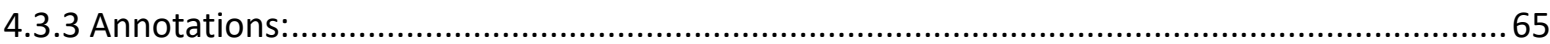

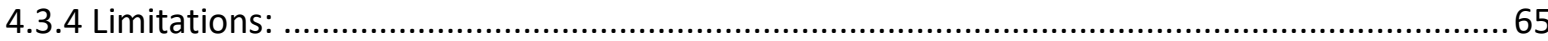

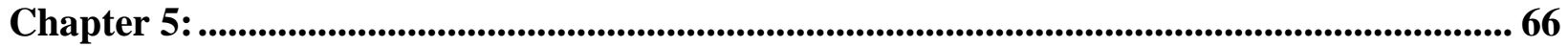

MAM Detection using Mask R-CNN: ...........................................................................66 
5.1 Introduction:

5.2 Three - Level Hierarchy for MAM detection: .............................................................................. 70

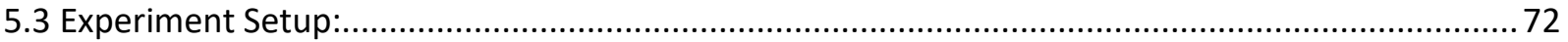

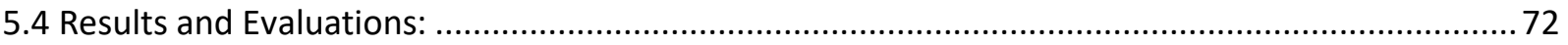

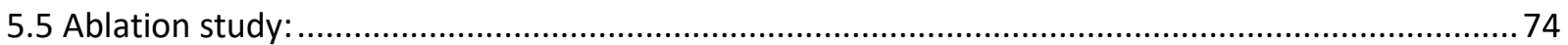

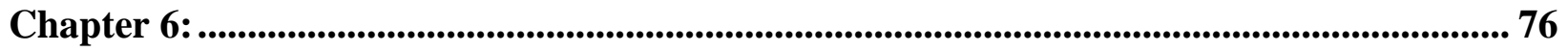

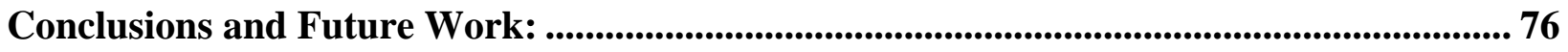

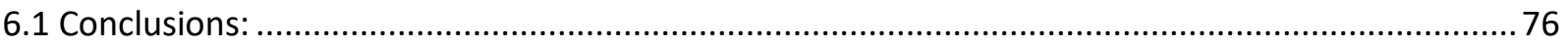

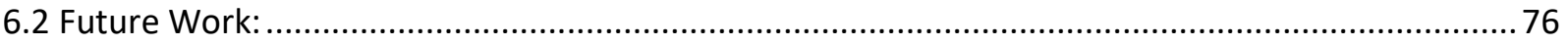

Bibliography ............................................................................................................................................... 79 


\section{List of Figures}

Figure 1.1: Figure illustrating the difference between classification, localization, detection, and

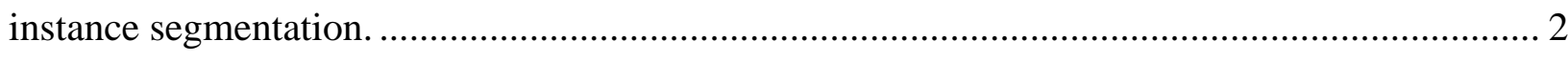

Figure 1.2: Difference between semantic segmentation (left) and instance segmentation (right).

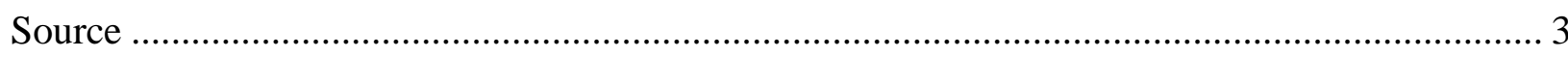

Figure 1.3: Drones in agriculture. Source ......................................................................... 5

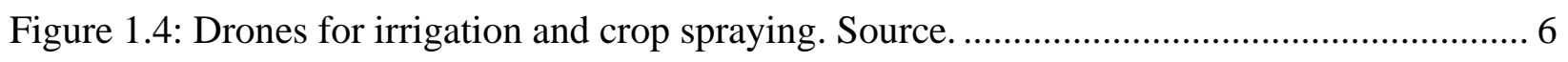

Figure 1.5: Drones for 3D mapping. Source ................................................................... 7

Figure 2.1: An example of Neural Style Transfer. (a) Content image, (b) Style image, (c)

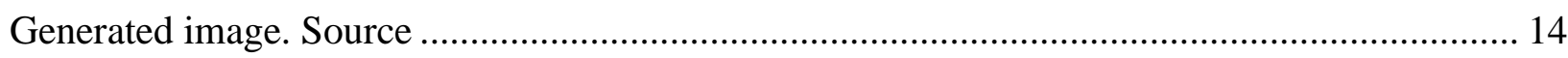

Figure 2.2: Convolutional Neural Networks for NST. The input image is passed through various filters and the number of filters increase as we proceed to the deeper layers. The size of the image will be decreasing due to down-sampling............................................................... 15

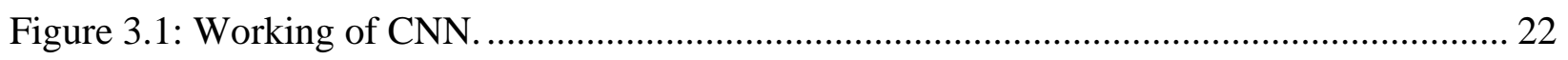

Figure 3.2: Selective search algorithm for object localization. Source .................................. 24

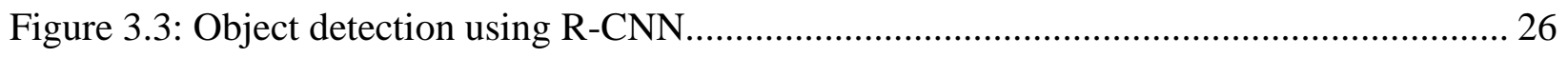

Figure 3.4: Intersection over Union. (a) Red bounding box is ground truth and the blue bounding box is the predicted bounding box. (b) Intersection of the bounding boxes. (c) Union of the

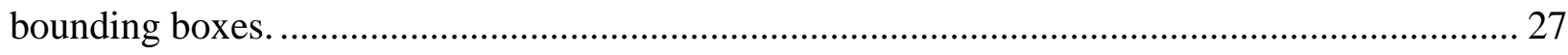

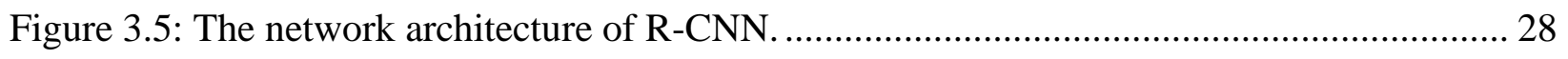

Figure 3.6: The network architecture of Fast R-CNN. ....................................................... 29

Figure 3.7: The network architecture of Faster R-CNN. ................................................. 30

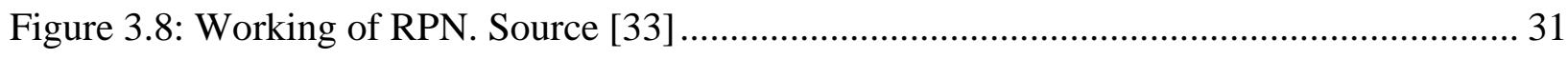

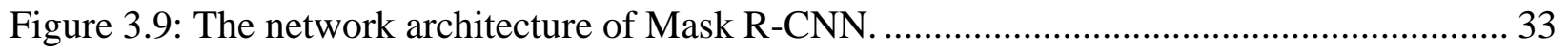

Figure 3.10: Pyramid architecture. (a) Pyramid of images. (b) Pyramid of feature maps. Source

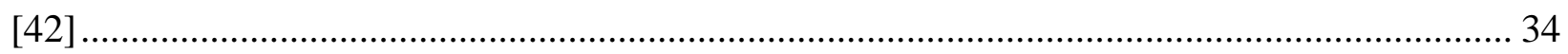

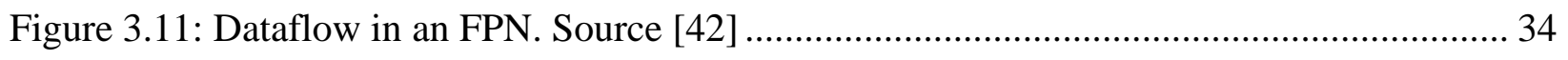

Figure 3.12: Working principle of ResNet. Source [43] .................................................. 36 
Figure 3.13: Images from CWFID. (a) RGB image of weed and crop plants. (b) Annotated image with the red colour being the weed plant and green colour being the carrot crop plant. Source [5]

Figure 3.14: Example images from the COCO type CWFID dataset...................................... 38

Figure 3.15: Types of images used for creating the synthetic data with 80 classes. (a) Foreground examples. (b) Background examples. ................................................................................... 39

Figure 3.16: Example image from the COCO type Synthetic dataset. ..................................... 40

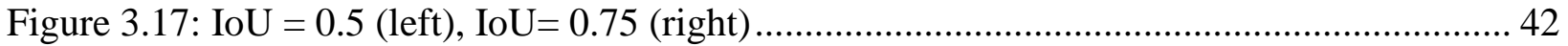

Figure 3.18: COCO type CWFID's Mask R-CNN results with Softmax threshold $=0.9$. The number on the top of the bounding box is the probability of the plant being a specific class like Crop_Plant or Weed_Plant.

Figure 3.19: Synthetic COCO dataset's Mask R-CNN results with Softmax threshold = 0.5. The number on the top of the bounding box is the probability of the plant being a specific class like

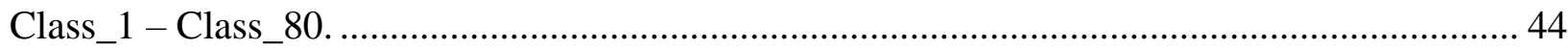

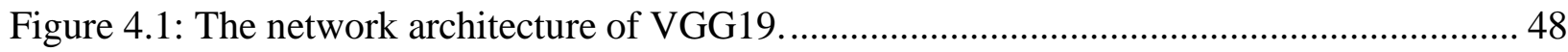

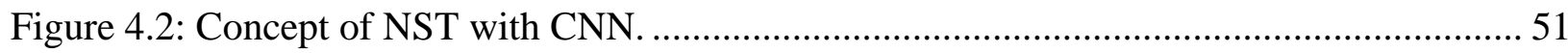

Figure 4.3: Computation of Style Matrix with 5 feature maps in a CNN layer. Source.............. 52

Figure 4.4: An example of localized style transfer. (a) content image, (b) Mask of the content image, (c) Generated image with a random style. Source [6] ................................................. 54

Figure 4.5: Sample images of Agriculture-Vision dataset's weed cluster class. (a) RGB image of the weed cluster, (b) Binary mask for the RoI. Source [6] ................................................. 55

Figure 4.6: Real-time MAM aerial images with manually marked MAM regions. .................... 56

Figure 4.7: Content, Mask and Style images used for our data augmentation using NST.......... 57

Figure 4.8: Procedure for mask augmentation using Albumentations.................................... 60

Figure 4.9: RGB (left) and binary mask (right) of the MAM aerial image. ............................. 61

Figure 4.10: Block diagram for binary mask generation for the marked aerial RGB images. ..... 64 Figure 4.11: Output of each block. (1) RGB image, (2a) Red channel of the RGB image, (2b) Grayscale of the RGB image, (3) Difference of 2a and 2b, (4) Difference image after denoising, (5) Binary image of the difference image, (6) Final mask after morphological reconstruction. .. 64 Figure 4.12: Example images from the Augmented MAM dataset. 65 
Figure 5.1: MAM infestation in the US (left) and areas in the east coast infected by MAM

(right). Source 67

Figure 5.2: Leaves (left) and fruits (right) of MAM. Source ................................................. 68

Figure 5.3: Spraying of herbicides for the entire field. Source ............................................. 69

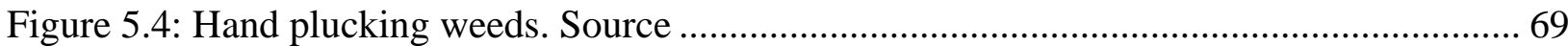

Figure 5.5: MAM weevil for biological control. Source ................................................. 70

Figure 5.6: Flow chart for our three-level hierarchy........................................................... 71

Figure 5.7: Augmented dataset's (section 4.3) Mask R-CNN results with Softmax threshold =

0.9. The number on the top of the bounding box is the probability of MAM's presence. .......... 72

Figure 5.8: Augmented dataset's (section 4.3) Mask R-CNN results with Softmax threshold =

0.5. With this threshold, we have false alarms such as car detection. .................................... 73

Figure 5.9: Mask R-CNN's performance on real-time data. .................................................. 75

Figure 6.1: Three-level hierarchy. (1) Forest level, (2) Trees level, (3) Plant level................... 77

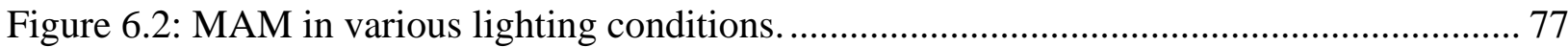

Figure 6.3: Weeds similar to MAM: Japanese Stilt grass (left) and Hedge Bindweed (right) ..... 78 


\section{List of Tables}

Table 1: Statistical comparison between other datasets and our synthetic dataset .................... 40

Table 2: Comparison of Mask R-CNN's performance on ground-level plant recognition image

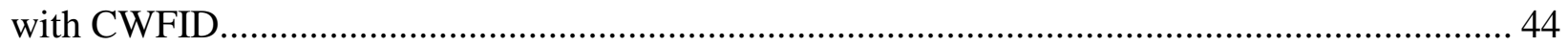

Table 3: ConvNet configuration of VGG19 ............................................................. 49-50

Table 4: Illustration of Global style transfer with and without colour transfer from the style

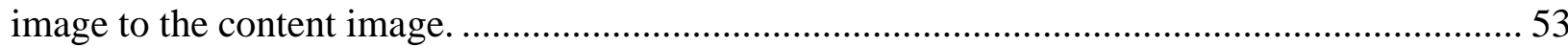

Table 5: Results of our NST data augmentation with VGG19 ........................................ 58-59

Table 6: Geometrical transformations on the RGB images and masks of aerial MAM data using

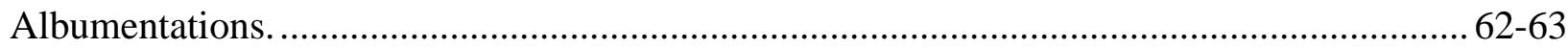

Table 7: COCO evaluation metrics for different RPN backbones........................................... 74 


\section{Chapter 1:}

\section{Introduction:}

Agriculture is one of the ancient and vital professions in the world. Over the millennia, humanity embraced various technologies like AI to boost productivity and efficiency in agriculture, thereby reduce negative environmental impacts. One of the greatest threats that the farmers face is low crop yields to weed infestations. Weed plants reduce the crop yield by $\sim 50 \%$ [1], which can have severe economic effects. Some of the most widely used methods to eliminate weed plants are using herbicides or robots powered with AI. The former option is inexpensive, but there is a chance that these herbicides might contaminate the crop plants, thereby posing a health risk. However, the latter option is a bit expensive, but it does not require human labour, and it will not cause any health risks. Hence, the usage of AI in agriculture has been prevalent nowadays.

\subsection{Motivation:}

Deep learning is a part of Machine Learning that mimics the human brain's workings in processing the data for object detection, recognition, and decision making. A convolutional neural network (CNN) [2] is a subclass of deep neural networks, generally applied to analyse visual images. CNNs are inspired by biological processes in which the connectivity pattern between neurons resembles the animal visual cortex's organization. CNNs use relatively fewer image preprocessing blocks compared to other image classification algorithms. Due to its promising results in visual recognition tasks, deep learning has been deployed into the agriculture sector. 


\subsubsection{Classification vs. Localization vs. Detection:}

One of the many enduring questions in the field of Computer Vision is, "What are the differences between Classification, Localization, and Detection?" Image classification is a relatively easy task compared to localization and detection. Image classification involves assigning a particular label to an image that gives us the details about that image's class. Object Localization involves creating a bounding box around the objects within an image, but it does not specify anything about the image's class. However, detection, on the other hand, involves creating a bounding box around the region of interest (RoI) and assigning a class to the different objects in the image. Hence, object detection is a combination of Image classification and localization. Often, the whole procedure for object detection is referred to as Object recognition. The figure below illustrates the difference between classification, localization, and detection.

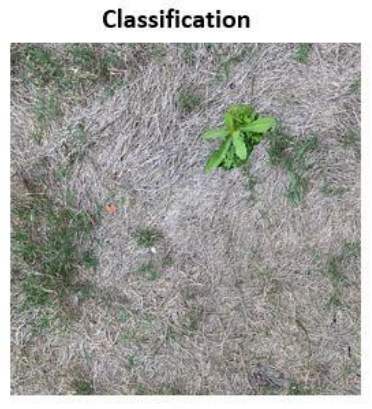

Species 1

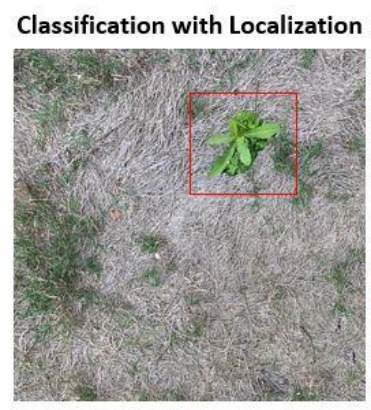

Species 1

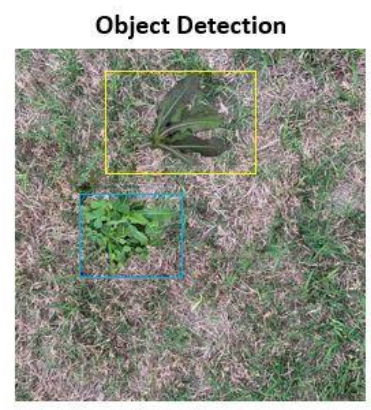

Species 1, Species 2

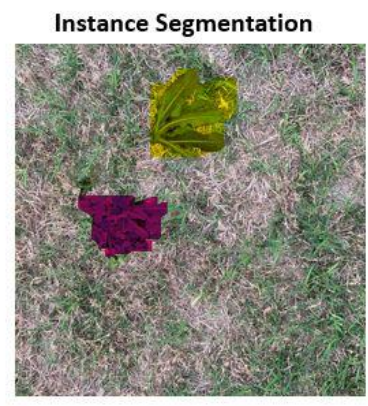

Species 1, Species 2

Figure 1.1: Figure illustrating the difference between classification, localization, detection, and instance segmentation.

\subsubsection{Object detection:}

Object detection is a vital computer vision task that deals with detecting objects of a particular class such as birds, people, vehicles in digital images and videos. Other applications of object detection include face detection, facial recognition, tracking objects, and video surveillance. Therefore, to gain a complete understanding of an image, instead of restricting our concentration to classifying images, we should expand it to precisely estimate objects' locations in a digital image. In this task, the input will be an image containing one or more distinguishable objects, and the output will be an image containing bounding boxes around those distinguishable objects and a 
label indicating the class of that object in the bounding box. The object recognition task can be further improved by adding Image Segmentation.

\subsubsection{Image Segmentation:}

Image segmentation involves drawing the bounding boxes around the objects in the input image at a pixel level. By doing so, we can differentiate many identical objects within a single input image. Image segmentation helps us analyse the image by breaking down the input image into segments containing the pixels of objects and pixels of the background. This pixel-wise segmentation will give us the object's shape instead of just creating a rectangular bounding box around the object. Image segmentation can be classified into two levels of granularity: Semantic segmentation and Instance segmentation.

- Semantic Segmentation: This is a process of detecting all the objects in an image and grouping them based on their categories. For example, all human beings are categorized into one category and all vehicles into another category.

- Instance Segmentation: This process is an advancement over semantic segmentation as it involves detecting the individual objects within the defined categories. For instance, it distinguishes each human-like adult, kid, and vehicle into cars, bikes.

The figure below provides us a better understanding of the difference between Semantic and Instance segmentation.

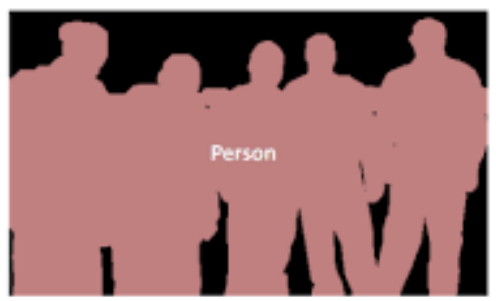

Semantic Segmentation

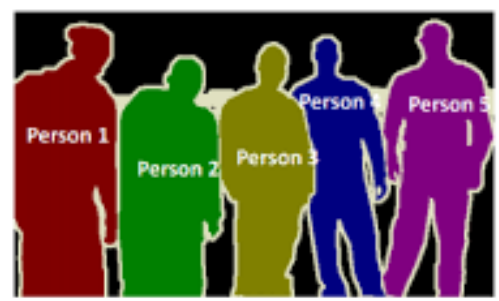

Instance Segmentation

Figure 1.2: Difference between semantic segmentation (left) and instance segmentation (right). Source ${ }^{1}$

\footnotetext{
${ }^{1}$ https://www.analyticsvidhya.com/blog/2019/04/introduction-image-segmentation-techniques-python/.
} 


\subsubsection{Data Augmentation:}

At times in deep learning tasks, lack of a sufficient dataset will create hindrances. One of the most straightforward solutions for this problem is to create a synthetic dataset. However, the synthetic images generated must be identical to the real-time images to get promising results. To do so, we used the Neural Style Transfer technique (NST) [3] and geometrical transformations. NST takes two images as input - a content image and a style reference image - and blends them so that the output image retains the core elements of the content image and acquires the style/texture of the style reference image. While the geometrical transformations, on the other hand, uses transformations on an image to obtain more images.

\subsection{5: Weeds in Agriculture:}

As per the Weed Science Society of America, a weed is defined as a plant whose growth is undesirable in a field, leading to ecological imbalances and economic loss. Occasionally, these weeds might also lead to health problems in human beings and animals. Some of the examples of weed plants are Poison Ivy, Tree of heaven. Taxonomically speaking, there is no actual significance for the word 'weed.' It is always subjective because a plant can be a weed in one context but not in another. Sometimes beneficial weeds are intentionally grown in the gardens. Often, weeds can grow invasively or aggressively outside the species' natural habitat. As per the natural enemies' hypothesis ${ }^{2}$, certain plants become very dominant when introduced into new ambiences due to a lack of fauna that feeds on them or lack fauna that competes with them.

Some of the drawbacks of having weeds in horticulture are:

- They compete with the crop plants for food, water, sunlight, and soil nutrients.

- They cause skin irritations to homo-sapiens and may cause discomfort in the animals' digestive tracts as some of the weeds contain thorns, burs, and even toxins.

- They can act as a host for several pathogens that might degrade the crop production,

\footnotetext{
${ }^{2}$ Positive correlation between plants and their natural enemies.
} 
- Weeds might damage other engineering works such as water sprinklers, drains, foundations.

- They cause degradation of lawns' aesthetic appearance, golf courses, and botanical gardens with their unappealing appearance.

It is essential to remove the weeds from the agricultural fields to prevent all the drawbacks mentioned above. The most prevalent methods to remove the weeds are using herbicides, lethal wilting, using mulch ${ }^{3}$. However, if the field area is vast, it is slightly harder to monitor the crop plants with limited human labour. Also, we need an expert to identify the species, and there aren't very many technicians that can do this job. So, this led to the usage of drones/UAVs to improve crop efficiency. Drone technology is an exceptional innovation with its uses in multiple domains. These drones can help the farmers to monitor crop spraying, irrigation mapping. UAVs aid in achieving so-called precision agriculture. In short, precision agriculture can be referred to as 'using fewer resources to grow more.' One of the limitations of using drones is that they are pricey. Nevertheless, as the population is increasing, the need for farming will only increase. The demand for drones in the agriculture market will increase in the future, leading to a decline in the price.

Farmers face many challenges that influence the success of their crops. Some of the challenges include climate change, soil quality, weeds, insect infestation. Farmers are tending towards UAVs to provide faster, reliable, and efficient results to address these issues.

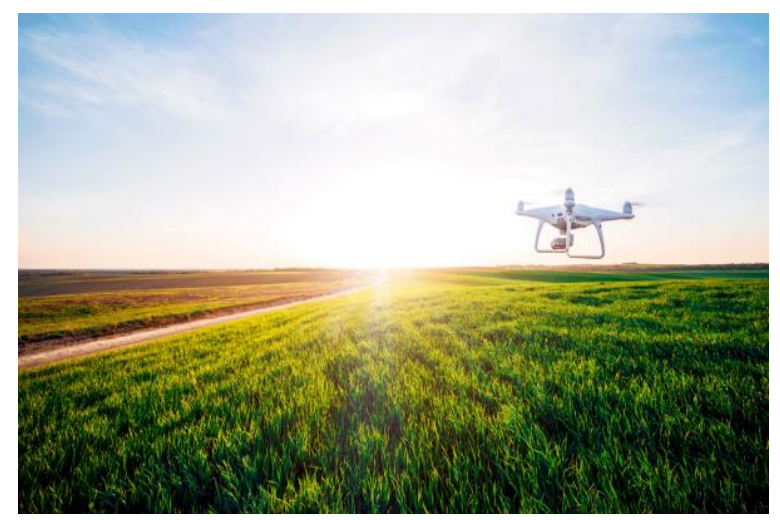

Figure 1.3: Drones in agriculture. Source ${ }^{4}$

3 material (such as decaying leaves, bark, or compost) spread around or over a plant to enrich or insulate the soil.

${ }^{4}$ https://www.greenbiz.com/article/making-drones-work-small-farmers. 


\section{Uses of drones in agriculture:}

Drones are usually equipped with IR cameras, GPS, programmable controllers, navigation units to collect data related to weeds, soil quality, and nutrients. This data can be used to get more precise information about the issues. Let us look at how drones answer some of the common issues in agriculture.

1. Crop Spraying: To maintain high crop yields, farmers need to spray herbicides, fertilizers, and pesticides. Conventional ways of spraying include using an automobile, aeroplanes, or even manual. Usage of these traditional methods is time-consuming and expensive. However, drones can solve this problem very quickly. The only additional requirement is they need to be equipped with reservoirs that can be filled with pesticides. This method is also helpful when we have to spray pesticides only to certain plants in the field instead of the whole field. This type of spraying is often referred to as spot spraying.

2. Irrigation Management: Like the previous functionality, drones can monitor crop plants to spot irrigation problems. Using drones, we can avoid water pooling or identify areas with less than the required moisture content. To do so, the drones must be equipped with thermal cameras.

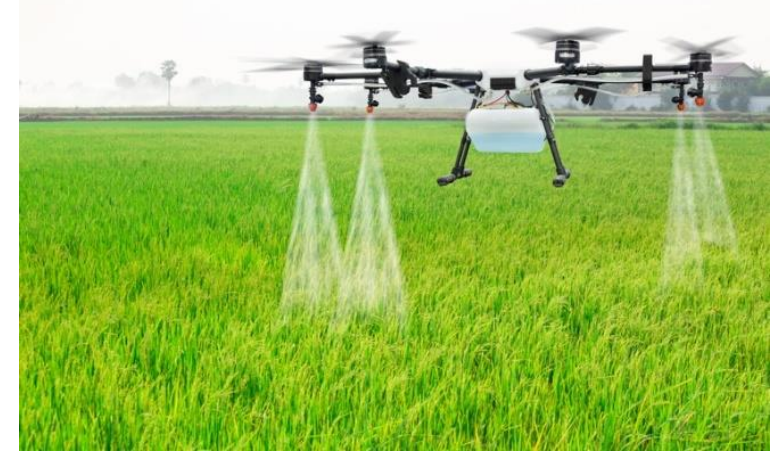

Figure 1.4: Drones for irrigation and crop spraying. Source ${ }^{5}$

\footnotetext{
${ }^{5}$ https://www.thomasnet.com/insights/drone-use-in-agriculture-is-soaring-to-new-heights/.
} 
3. Seed Planting: Drones are not yet widely used to solve this problem, but experiments are still going on for the effective use of drones to shoot seeds into ploughed soil.

4. Soil Analysis: Soil analysis includes identifying nutrient requirements, soil quality analysis, and identification of dead/barren lands. The drones must be equipped with instruments that can capture the 3D maps to obtain the soil information.

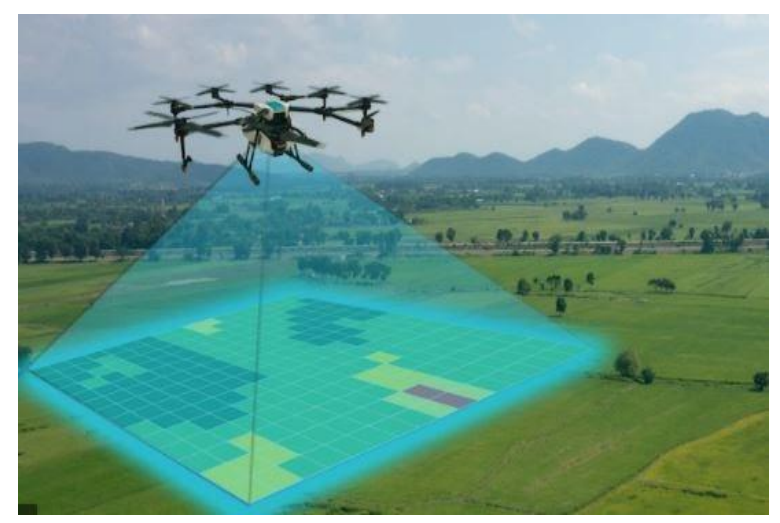

Figure 1.5: Drones for 3D mapping. Source ${ }^{6}$

5. Crop Surveying: This is by far the essential application of using drones in the field of agriculture. Traditionally, plane images and satellite images are used to get farm images. Nevertheless, obtaining information from satellites can be outdated. With NIR drones, plant health can be easily determined by calculating the amount of light absorbed.

As deep learning models do an excellent job identifying most common objects like cars, people, our objective is to use the very idea to detect the plant species using drone images.

In this thesis, our primary focus is one weed called Persicaria Perfoliata or Mile-A-Minute (MAM). ${ }^{7}$

\subsection{Deep Learning for weed recognition:}

Deep learning mimics the human brain's workings for processing the data using multiple network layers to extract higher-level features from the raw input images and hence used for many

\footnotetext{
${ }^{6}$ https://pilotinstitute.com/drone-mapping/.

${ }^{7}$ http://nyis.info/invasive_species/mile-a-minute/.
} 
visual recognition tasks. In the field of agriculture, all the objects (plants and weeds) will be mostly green in colour (green on green), so object recognition (in our case, species identification) is relatively more complicated as most of the object recognition algorithms use colour, fill, texture and size for object recognition (refer to section 3.1). To eliminate the false positives problem, we propose a three-level hierarchy (refer to section 5.1) for identifying weeds using UAV images. The hierarchy is as follows:

1. Forest level (very high-altitude images)

2. Tree level (low-altitude images)

3. Leaf level (ground-level images)

\subsection{Problem Statement:}

In this thesis, we report our ground level and low altitude image studies. Our key focus is on the following problems.

1. Leaf level: Weed plant species recognition using Mask R-CNN.

2. Tree level: Synthetic Dataset creation using Neural Style Transfer and geometrical transformations.

3. Mask R-CNN's performance analysis with the augmented data produced in statement 2.

\subsubsection{Ground level weed plants recognition using Mask R-CNN:}

In this study, we used Mask R-CNN [4] to distinguish the weed plants among the crop plants. To achieve this task, we used the Crop/Weed Field Image Dataset (CWFID) [5]. However, this dataset's limitation is that it classifies all the weed plants into a single class and the crop plants are of single species. To expand our study, we used 80 foreground images of weed plants to create a synthetic dataset. We labelled them as class 1 through class 80 . 


\subsubsection{Synthetic Dataset creation using Neural Style Transfer and Geometrical transformations:}

In this part of our study, we primarily focused on a particular invasive weed plant called MileA-Minute (MAM). We aim to detect MAM plants using Unmanned Aerial Vehicles (UAVs). As we are using a supervised model for our MAM predictions, the number of data points is proportional to the number of trainable parameters of a model, which is proportional to the complexity of the task that needs to be achieved. Hence, we need to have an adequately annotated dataset to use Mask R-CNN or any other supervised learning network. Since we could get only a few real-time UAV images of MAM, we used Neural Style Transfer (NST) technique to create our synthetic dataset. For the content images, we used the Agriculture Vision dataset [6], and for the style reference, we used our UAV images. We used VGG 19 [7] architecture to accomplish our NST task. Due to this dataset's limitations, we have created one more synthetic dataset using geometrical transformations. We have used transformations such as flips, rotations, and grid distortions to achieve the data augmentation task in the latter dataset.

\subsubsection{MAM detection using Mask R-CNN:}

In this section, we focused on detecting MAM regions using UAV images. Being an aggressive invasive plant, MAM can decrease the crop plant's ability to photosynthesize ${ }^{8}$ and potentially kill the plants by smothering them with their weight (refer to section 5.1). So, detection of MAM zones is vital for the crop fields with MAM infestation. Using the low-altitude images mentioned in section 1.3.2, we trained Mask R-CNN to recognize the MAM zones and reached an AP50 97\% for bounding box and AP50 92\% segmentation.

\subsection{Contribution:}

- We created a synthetic dataset with 80 foreground images, thereby classifying them into 80 classes, and fed the dataset to Mask R-CNN.

\footnotetext{
${ }^{8}$ The process of synthesizing food from sunlight, $\mathrm{CO}_{2}$ and $\mathrm{H}_{2} \mathrm{O}$
} 
- We have created synthetic datasets using a localized style transfer technique and geometrical transformations technique.

- We tested Mask R-CNN's performance on the real-time augmented data.

\subsection{Dissertation Structure:}

The remainder part of this thesis is organized as follows:

- Chapter 2 focuses on the literature review about detectron2 and style transfer.

- Chapter 3 reviews the experimental setup and dataset details used for ground-level weed recognition using Mask R-CNN.

- Chapter 4 focuses on various data augmentation techniques for aerial images, including NST and geometrical transformations

- Chapter 5 presents our approach to detect MAM using Mask R-CNN.

- Chapter 6 focuses on conclusions and future work. 


\section{Chapter 2:}

\section{Literature Survey:}

\subsection{Related Work:}

In the recent past, many deep learning models were introduced for object recognition tasks. However, when it comes to the agriculture domain, the object recognition task is challenging as the weed plants and crop plants might have the same colour, texture, fill, and size. Classification is a relatively easy task compared to the recognition tasks at lower altitudes, to be precise on a leaf level. There are many public datasets at the leaf level for species identification [8], disease prediction in one [9], or more species [10] [11]. However, when it comes to real-time applications, we need to focus on datasets at the plant level. There have been many advances in these plantlevel classification tasks. Most agriculture datasets focus on diseased crop identification. Very few datasets like DeepWeeds [12] focus on weed plants that grow among the crop plants. However, DeepWeeds focus on eight different species that are native to northern Australia, and it does not provide any data about the localization of the plants, thereby restricting it to classification tasks. The lighting of the image also plays a crucial role in agriculture tasks along with the quality. Most of the mentioned datasets have a single lighting condition. Carrot-Weed [13] is a dataset that provides images at different lighting conditions, but as the name suggests, the crop images are restricted to carrot plants. Specific datasets like Plant Phenotyping [14], Plant Seedling dataset [15], and others [16] provide us information about the vegetation areas, but the limitation of these 
datasets is that the background is soil or stones instead of other plants. Even at the plant level, without proper annotations indicating specific plant species' location, it is hard to recognize the species among several plants. Recent advances in the field of object detection lead to the collaboration of agriculture and deep learning fields to achieve precision agriculture [17] [18]. Usage of CNNs for detecting weeds among certain plants like turfgrass [19], ryegrass [20], soybeans [21] was proven to be a suitable method for weed management. Along with the supervised models, unsupervised models with minimal labelling have also been in use for the weed detection [22]. In this thesis, we have created a synthetic dataset of 80 weed species with more than one class per image to expand our study on Mask R-CNNs performance on weed recognition.

For our aerial image study, we focused on recognizing MAM using UAV images. Due to the ever increase in population, the demand for growing food is expected to increase despite limited farmlands. To grow more food with fewer resources, farmers are now adapting the so-called Precision Agriculture. Precision agriculture involves modern technology usage, including but not restricted to drones and dusters for crop management. Although drones are not currently allowed for every agricultural need (such as carrying harmful substances) due to Federal Aviation Administration (FAA) regulations, dusters ${ }^{9}$ can still be used for crop management as they fly at very low altitude (10 foot above the ground). However, dusters are far more expensive than drones. However, drones can be used for crop management following the FAA regulations. Recognizing the agriculture patterns such as weed recognition can be very challenging at a very high altitude. Using the multispectral images taken by drones, we can detect the plant species. There are very few datasets [6] [23] created for pattern recognition in agriculture. Hence, we propose a three-level hierarchy (forests, trees, and leaves) for confirming the presence of MAM in a given field, with the forest being the high altitude, trees being the low-altitude, and leaves being the ground-level images. In this thesis, we analysed the performance of Mask R-CNN at low altitude and ground levels. As there are no specific datasets dedicated to MAM recognition, we created synthetic data using NST and standard augmentation techniques.

\footnotetext{
${ }^{9}$ Usage of aerial vehicles for dusting the crops
} 


\subsection{Neural Style Transfer:}

\subsubsection{Introduction:}

Neural Style Transfer (NST) is an illustration of image stylization within the field of NonPhotorealistic Rendering (NPR). NPR is a subset of Computer Graphics (CG) focusing on enabling a wide range of expressive styles for digital art. Unlike conventional CG, NPR does not focus on photorealism $^{10}$. Due to its inspiration from other artistic modes such as animations, drawing, painting, NPR is often used in movies and video games. The first two illustration-based style transfer algorithms were based on patch-based texture synthesis algorithms called image analogies [24] and Image Quilting [25].

Texture synthesis is generally used to fill in holes in images like inpainting ${ }^{11}$ or expand the small pictures. Texture synthesis algorithmically constructs a large image from a small digital sample. There are multiple techniques to achieve this goal. Some of the techniques available are patch-based texture synthesis, pixel-based texture synthesis, tilting, stochastic texture synthesis. Early style transfer algorithms are based on patch-based texture synthesis. Patch-based Texture synthesis is faster and effective compared to pixel-based texture synthesis because the patch-based texture synthesis creates a new texture by replicating and stitching other textures at various offsets. Image analogies, Image quilting, and graph-cut textures are some of the best patch-based texture synthesis algorithms.

- Image Analogies: It is a process of creating an image filter from training data. Texture mapping is used for texture synthesis from an example texture image. For a given image pair containing an image and an artwork of that image, by analogy, a transformation can be learned to create new artwork from another image.

- Image Quilting: A new image is synthesized by stitching small patches of existing images. It can be used only for a single style.

\footnotetext{
${ }^{10}$ a style of art characterized by the highly detailed depiction of ordinary life with the impersonality of a photograph.

${ }^{11}$ Inpainting is a conservation process where damaged, deteriorating, or missing parts of an artwork are filled in to present a complete image. [Wikipedia]
} 
Image Quilting was later used for Texture transfer by rendering an object with a texture that is taken from another object [25].

Nowadays, deep learning and neural network approaches are proven to be fast and powerful. The publication of the article "A Neural Algorithm of Artistic Style" in 2015 [3] laid the foundation for the usage of Convolutional Neural Networks (CNNs) to the problem of style transfer in image processing. Style transfer is a procedure that involves transferring the style of one image to another while preserving the content of the target image. The figure below is an illustration of NST.

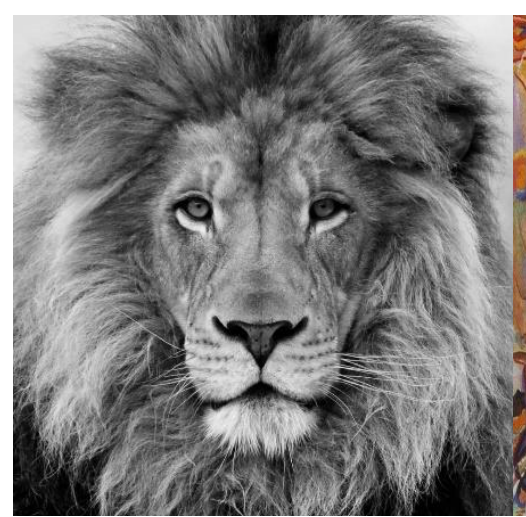

(a)

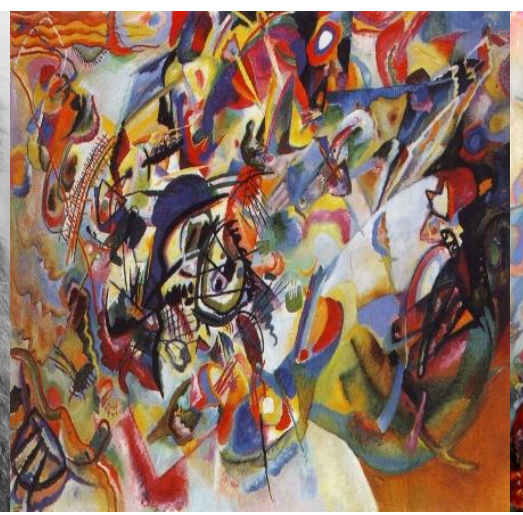

(b)

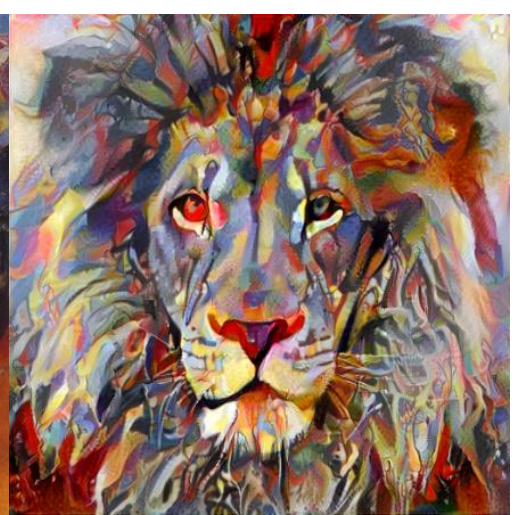

(c)

Figure 2.1: An example of Neural Style Transfer. (a) Content image, (b) Style image, (c) Generated image. Source ${ }^{12}$

CNNs usually consist of multiple layers of computational units. Each computational unit layer can be defined as a collection of image filters that can extract the desired features from the image that is fed as an input. The output of each layer will be a divergent representation of the filtered input images called feature maps. When we train a $\mathrm{CNN}$ for an object recognition problem, the feature maps usually care about the image's actual content compared to its pixel values. In general, the lower layers capture the low-level information such as precise pixel values, whereas the higher layers, on the other hand, capture the high-level information such as the arrangement of an object in the image, which is the content itself. Often, the higher layers are referred to as content representation. With this idea as a baseline, NST is developed. While performing the style transfer, instead of training a neural network, we start with a blank image and cost function and alter each pixel iteratively to reduce the cost function. In short, instead of updating biases and weights while

\footnotetext{
12 https://www.wikiart.org/en/wassily-kandinsky.
} 
training a neural network, we update the image itself. NST uses a pre-trained CNN. The commonly used jargon for NST are:

- Content image: the image that gets the transferred style.

- Style image: the image whose style is transferred.

- Generated image: the final image that contains the transformed image.

The basic formulation is the content image is fed into a $\mathrm{CNN}$, and the network activations are sampled according to the network architecture. Then the style image is fed through the same layers, and the network activations are sampled from the early to middle layers of the network. The ultimate goal of NST is to create a generated image that shows the content of the content image applied with a style of the style image. The image below is an example of an NST formulation.

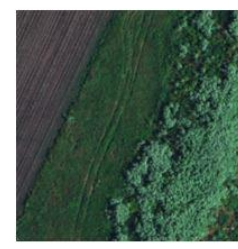

Content image

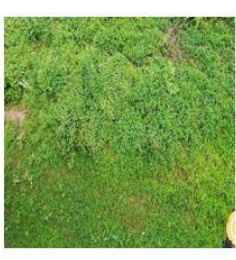

Style image

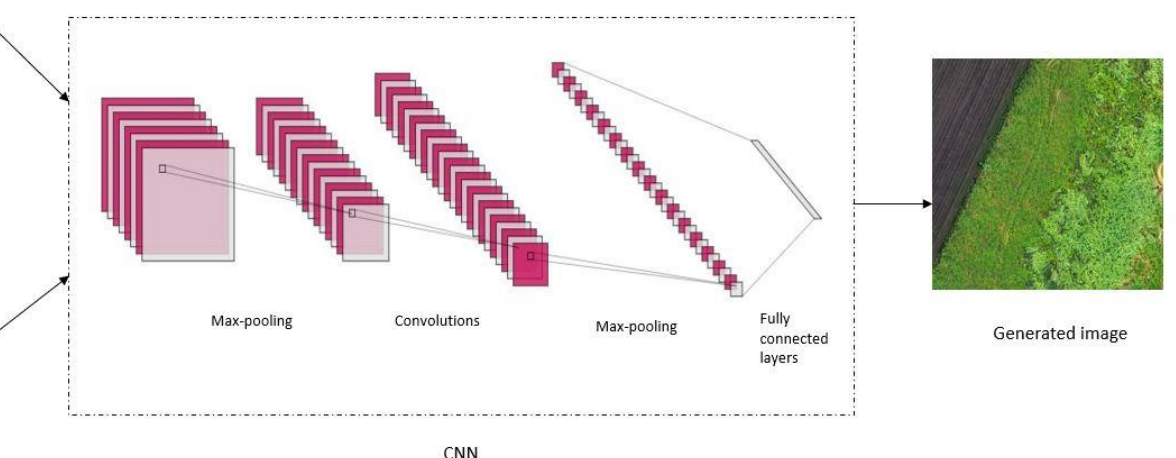

CNN

Figure 2.2: Convolutional Neural Networks for NST. The input image is passed through various filters and the number of filters increase as we proceed to the deeper layers. The size of the image will be decreasing due to down-sampling.

Further development in this field includes using generative models such as Spatial GAN [26] for texture synthesis. The Spatial GAN method uses fully unsupervised GANs. Its extension PSGAN [27] uses unsupervised learning to learn both periodic and aperiodic images from a single image or massive datasets. 


\subsubsection{Style transfer vs. localized style transfer:}

Majority of the style transfer applications require the transfer of the style to the entire image. Nevertheless, at times we need a partial-image style transfer called localized style transfer ${ }^{13}$ [28] [29]. In this dissertation, we have used a localized style transfer technique to create a synthetic dataset. This localized style transfer involves selecting specific regions in the input image that needs a style transfer (usually by using masks) and then applying the style transfer to that masked region, thereby leaving the unmasked region unaltered.

\subsubsection{NST for Agriculture dataset:}

Lack of a properly annotated dataset can affect the overall performance of the object detection models. This problem is more challenging for agriculture tasks such as weed recognition and crop recognition due to a lack of public datasets. Compared to standard data augmentations techniques like flipping and rotating, NST has proven to provide better accuracy when it comes to classification tasks [30]. We can create a synthetic dataset that resembles the original crop images to address our lack of proper annotated dataset problem by using NST. In this thesis, we have generated a synthetic dataset using some aerial images as content images and MAM images as style images.

\subsection{Detectron2:}

\subsubsection{Introduction:}

Object recognition is one of the fundamental visual recognition tasks in the field of Computer Vision. Over the past few years, numerous implementations of object recognition algorithms have been made on various open-source platforms. Deep learning architectures like Convolutional Neural Networks (CNNs) can automatically learn object features by analysing thousands of

\footnotetext{
${ }^{13}$ https://github.com/cysmith/neural-style-tf.
} 
training images to identify various objects in an image. Traditionally, there are two approaches to recognize objects using deep learning.

- Training a model from scratch: In this approach, we need numerous labelled training images. We also must set up layers and weights to design a model that can accurately learn the training data features and provide precise predictions on the testing data.

- Transfer learning: In this approach, we use a procedure called fine-tuning. This approach is less time-consuming and is widely used in Deep Learning applications because we feed a new dataset with unknown classes to an existing model such as VGG 16 [7], MobileNet [31], that has already been trained on several thousands of images.

Detectron is an open-source software that implements most state-of-the-art algorithms for Object detection and classification. It is implemented using Caffe $2^{14}$, a deep learning framework. Detectron has become one of the most used open-source platforms by Facebook AI Research (FAIR $)^{15}$. However, as Detectron is built on the Caffe 2 framework, its purpose has been restricted to production use. Caffe2 primarily focuses on scalable systems and cross-platform support for applications that involve large-scale object detection and classification. For the research flexibility and applicability, PyTorch is a preferred choice.

\section{PyTorch vs. Caffe2:}

Caffe2 is more developer friendly than PyTorch when it comes to model deployments on various platforms like Android, Raspberry Pi, iOS. Caffe2 has the upper hand in deploying the models over PyTorch because Caffe2 can run on any platform once coded. Also, most of the networks written in PyTorch can be deployed in Caffe2.

However, when it comes to flexibility issues involved in research, such as parameter alterations, model changes, and debugging, PyTorch has the upper hand. Due to PyTorch's dynamic nature, it

\footnotetext{
${ }^{14} \mathrm{https}$ //developer.nvidia.com/blog/caffe2-deep-learning-framework-facebook.

${ }^{15}$ https://ai.facebook.com/.
} 
can overcome TensorFlow's limitations and Keras ${ }^{16}$ as well. Hence, for the research applications, PyTorch will be a better option.

Facebook's research team developed Detrectron $2{ }^{17}$, a second generation of the Detectron library using the PyTorch framework to support research and production applications. Detectron2 is very flexible and provides fast training on single or multiple GPU servers. Detectron $2^{18}$ is a modular design that allows us to plug custom module implementations into any part of an object detection system.

\subsubsection{Data Loader, Models, and Data Augmentation:}

Data Loaders are the components that take raw information from the provided datasets and process them into a format that the model requires. Detectron 2 has a built-in data loader, but it also allows the user to define their own data loader (custom data loader). Throughout this thesis, we used the existing data loader provided by Detectron2. To understand the data loader's working, we need to understand the functionality of build_detection_\{train, test $\}$ loader, the two functions provided by Detectron 2 to create a data loader from a chosen config. It loads a list containing a registered dataset's lightweight format such as PASCAL VOC, COCO [32] and performs a few pre-processing tasks like augmentation and memory allocation. Each dictionary in this list is mapped by using a function called mapper. This mapper function transforms the dataset's lightweight representation into a model-ready representation by applying augmentation, allocating memory, and performing torch tensor conversions. This output is batched and fed to a model.

Data Augmentation ${ }^{19}$ is a procedure used to increase data images' number by using slightly altered copies of existing data. Detectron2's data augmentation allows us to augment multiple data types such as images with masks and images with bounding boxes together. It also facilitates the users to add custom new data types such as rotated bounding boxes and manipulate the operations

\footnotetext{
16 https://keras.io/.

${ }^{17}$ https://github.com/facebookresearch/detectron2.

${ }^{18} \mathrm{https}$ ://detectron2.readthedocs.io/en/latest/.

${ }^{19}$ https://en.wikipedia.org/wiki/Data_augmentation.
} 
applied by augmentations. We can also use a sequence of geometrical augmentations. For more advanced applications, we can customize the transform strategy by performing a geometrical inversion of the transform, new data type addition such as coordinates, masks, polygons, and much more.

Detectron2 includes the following object detection models: Faster R-CNN [33], Mask R-CNN [4], RetinaNet [34], Cascade R-CNN [35], and Panoptic FPN [36], and many more to come. Detectron2 uses GPU for the entire training pipeline, thereby making it faster than Detectron. Each dict (dictionary) obtained from the data loader corresponds to a single image. The Key points required are model-dependent. Based on our requirements, we can use the model for training or inference. If we use the model in training mode, all the training statistics are stored. However, if we want to perform inference with an existing model, we have to use DefaultPredictor wrapper to perform pre-processing, model loading, and batch operations. The model's input is a list of dictionaries containing the keys like image channel information, desired output's height and width, instances such as masks and key-points, bounding box information. The output will be a dictionary that includes all the losses.

\subsubsection{Training and Evaluation of models:}

One of the most useful features of Detectron2 is that it allows the users to customize their training loop. Once the model and data loader are ready, we can write our training loop by using the tool provided by PyTorch, thereby giving the users full control over the training logic. However, we can also use the standard trainer abstraction that simplifies the training process as well. DefaultTrainer is the most often used trainer abstraction compared to SimpleTrainer ${ }^{20}$. The DefaultTrainer allows the users to perform all the default configurations for the optimizer, learning rates, checkpoints, evaluations, and logging. Whereas the SimpleTrainer, on the other hand, allows the user to perform minimal training loops for single-optimizer, single-data-source, and singlecost. During the training procedure, all metrics are stored in a centralized repository called

\footnotetext{
${ }^{20}$ https://detectron2.readthedocs.io/en/latest/.
} 
EventStorage. The users can access these logs to get information about accuracy and other parameter details.

Although training a model is vital, understanding how well the model predicts unseen images is also challenging. It is important to check if the model is merely memorizing the training data or is it generalizing the new dataset. Detectron2 has a built-in DatasetEvaluator that computes metrics using standard dataset APIs. All these evaluators are dataset - specific as they use the dataset's official API. For a custom dataset that follows Detectron2's dataset format, we can use COCOEvaluator for bounding box detection, instance segmentation, key-point detection and SemSegEvaluator for semantic segmentation. Finally, the models need to go through export procedures to become deployable artifacts. Deployment can be done using Tracing or Scripting or by using Caffe2Tracer. 


\section{Chapter 3:}

\section{Weed recognition using Mask R-CNN:}

\section{1: Introduction to Mask RCNN:}

Over the past few years, computers' usage in identifying digital images' properties, thereby giving them a human perception, has been increasing. Usage of images has been increased with the advent of smartphones. This attempt to give the computer a vision led to substantial growth in the usage of images and videos. For instance, there are about a billion videos that are watched on YouTube daily. To better understand the internet data, it is necessary to make a computer see and understand the image and its contents. Using Computer Vision ${ }^{21}$, we can perform the tasks like feature extraction, image classification, image classification with localization, object detection, object segmentation, and style transfer.

\section{A brief history of Convolutional Neural Network:}

Just like a human brain, CNN works by scanning a digital image from top to bottom or left to right to extract features. However, CNNs are not restricted to 2D images. They can be applied to

\footnotetext{
${ }^{21}$ Computer vision is an interdisciplinary scientific field that deals with how computers can gain high-level understanding from digital images or videos. From the perspective of engineering, it seeks to understand and automate tasks that the human visual system can do. [Wikipedia]
} 
1D and 3D data as well. Convolution is referred to as a procedure that applies filters to the given image and results in an activation function as output. Redundant application of the same filter will yield a map called feature map. The filters applied can be handcrafted to achieve an objective required by the user. Some of the examples of filters include edge detectors, line detectors, shape detectors.

To understand how image classification is done using a CNN, we need to dig deep into a CNN's working. The CNNs take in a multi-colour channelled image and pass it through a series of filters that detect features and provide us the output feature maps. These feature detectors detect various features like different shapes, colours, and edges. CNNs allow multiple convolutions parallelly. With all these filters, the CNNs will learn to see different image features after training it with many images. As CNNs allow multiple channels, each image may have three colour channels: RGB (red, green, and blue). The filters applied to the input images must allow the same number of channels as their inputs, called depth. Once the filter is applied to an image, a dot operation is performed, thereby yielding a single scalar value as an output. As CNNs allow multiple convolutional layers, the first layers usually extract the low-level features, but as we proceed to deeper levels, the layers can extract high-level information such as people, animals, vehicles. The most often used activation function in CNNs is ReLU, as it is simple to use and yields better performance most of the time. ReLU stands for Rectified Linear Activation function. There are other activation functions such as hyperbolic tangent and sigmoid but, their performance is compromised when we have multiple layers as they lead to vanishing gradient problem [37].

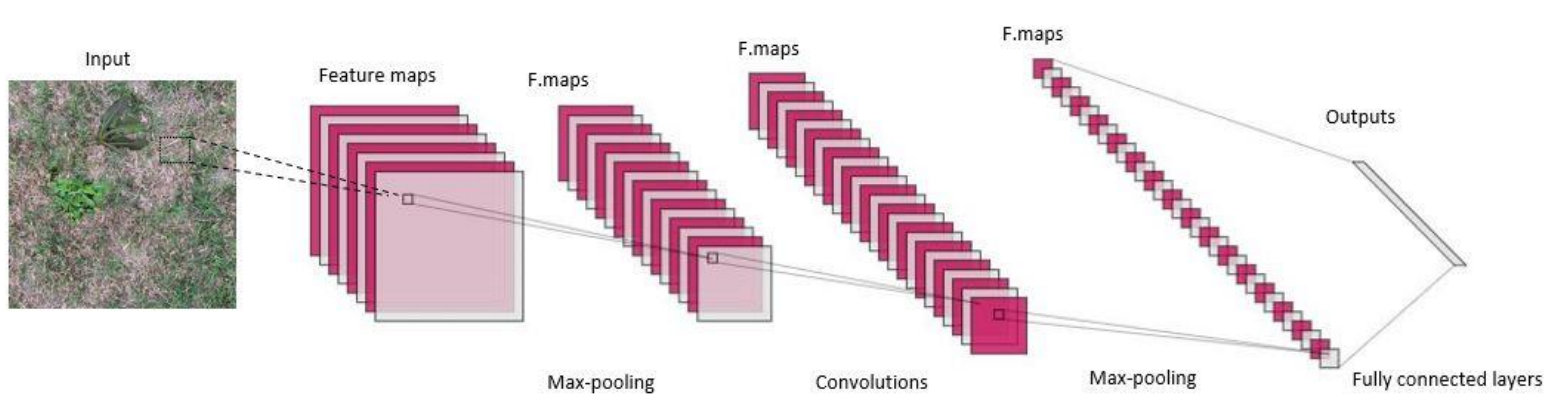

Figure 3.1: Working of CNN. 
As the convolutional layers summarize all the features present in the input image, we then apply pooling to the convolution layer output. The output feature maps are usually location-sensitive when it comes to detecting features in an input image. To address this sensitivity issue and make the feature maps more robust to the position change in an image, we use down-sampling, which summarizes the features of the feature maps in patches. The commonly used pooling methods are max pooling, min pooling, and average pooling. In max and min pooling, we summarize the most activated and least activated feature presences, respectively, but in average pooling, we average the feature presences. By doing so, we can achieve Translational Invariance, meaning even if the input image is rotated, resized, viewed in alternative brightness, we have no variance. After getting a pooled layer, we flatten it and feed it fully connected ${ }^{22}$ neural networks. We use an activation function for the final output layer, such as sigmoid or Softmax, for binary and multi-class classification. Although CNN performs well for image classification and single object recognition, it fails when there are multiple objects in the same image due to visual interference. This drawback led to the advent of Region-based CNN.

\section{R-CNN:}

To detect multiple objects in given image, R-CNN [38] is introduced. It uses selective search algorithm to generate a region proposal and help detect objects in an image. Region proposals can be defined by considering the altering textures, colours, scales, or even enclosures. To deal with the object localization issue, we can use the sliding window technique. This sliding window technique uses different window sizes to locate objects in a digital image. Hence, this procedure is often referred to as Exhaustive search. Exhaustive search is computationally expensive as we have to search for objects in thousands of windows, even for a relatively small image. Many improvisations, such as using different window sizes, were proposed later for this method, but none effectively improved computational efficiency. R-CNN uses the selective search algorithm to address the object localization problem. The selective search algorithm is a combination of Exhaustive search and segmentation. R-CNN segmentation is a method of separating different objects in an image by assigning different colours to every new object in that image.

\footnotetext{
${ }^{22}$ FC layers are generally used to identify the global configurations of the features.
} 


\section{Selective search algorithm:}

1. Generation of initial sub-segmentation of the input image as described in "Efficient Graphbased Image Segmentation" [39]

2. Use the Greedy algorithm to combine similar regions.

\section{Greedy algorithm:}

1. In the given set of regions, choose the most similar regions.

2. Combine the similar regions.

3. Repeat it for multiple iterations.

4. Get the object location from the segmented regions.

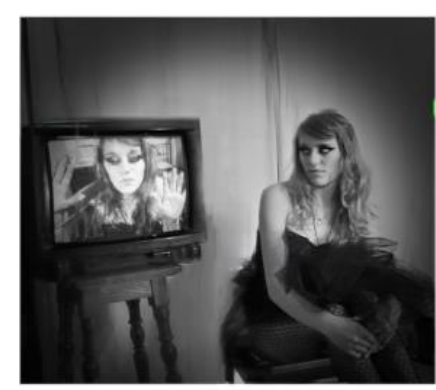

Input Image

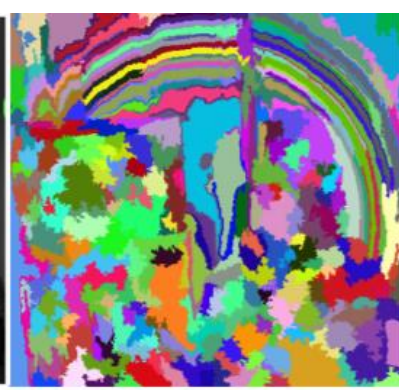

Initial Segmentation

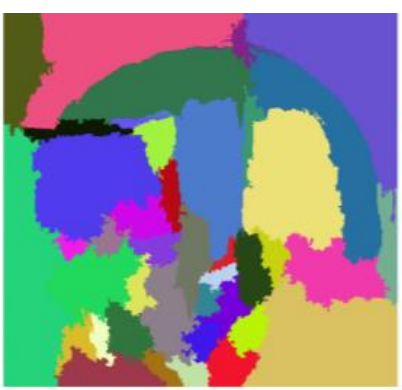

After some iterations

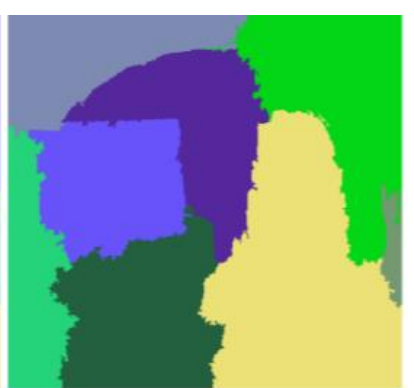

After more iterations

Figure 3.2: Selective search algorithm for object localization. Source $e^{23}$

In the segmentation part, to obtain more extensive segmentation results from the initial small segmentations, four types of similarities are considered: Colour similarity, Texture similarity, Fill similarity, and size similarity.

- Colour similarity: The similarity is found by combining the bins taken from the histogram of each channel in the given image by using the following expression.

\footnotetext{
${ }^{23}$ https://www.geeksforgeeks.org/selective-search-for-object-detection-r-cnn/.
} 


$$
S_{\text {colour }}\left(r_{i}, r_{j}\right)=\sum_{k=1}^{n} \min \left(c_{i}^{k}, c_{j}^{k}\right)
$$

$\mathrm{c}_{\mathrm{i}}^{\mathrm{k}}, \mathrm{c}_{\mathrm{j}}^{\mathrm{k}}$ are the $\mathrm{k}^{\mathrm{th}}$ value of the histogram bin of region $\mathrm{r}_{\mathrm{i}}$ and $\mathrm{r}_{\mathrm{j}}$, respectively.

- Texture similarity: The texture similarity is obtained by calculating 8 Gaussian derivatives of the ima: Chapter 3: Weed recognition using Mask R-CNN ;togram is extracted by using the following formula.

$$
S_{\text {texture }}\left(r_{i}, r_{j}\right)=\sum_{k=1}^{n} \min \left(t_{i}^{k}, t_{j}^{k}\right)
$$

$\mathrm{t}_{\mathrm{i}}^{\mathrm{k}}, \mathrm{t}_{\mathrm{j}}^{\mathrm{k}}$ are the $\mathrm{k}^{\mathrm{th}}$ value of histogram bin of region $\mathrm{r}_{\mathrm{i}}$ and $\mathrm{r}_{\mathrm{j}}$, respectively.

- Fill similarity: The following formula is used to calculate how well the two regions fit with one another. The condition is, if the regions fit, they should be merged else not.

$$
\mathrm{S}_{\mathrm{fill}}\left(\mathrm{r}_{\mathrm{i}}, \mathrm{r}_{\mathrm{j}}\right)=1-\left(\operatorname{size}\left(\mathrm{BB}_{\mathrm{ij}}\right)\right)-\operatorname{size}\left(\mathrm{r}_{\mathrm{i}}\right)-\operatorname{size}\left(\mathrm{r}_{\mathrm{j}}\right) / \operatorname{size}(\mathrm{image})
$$

Size $\left(\mathrm{BB}_{\mathrm{ij}}\right)$ is the size of the bounding box around $\mathrm{i}$ and $\mathrm{j}$.

- Size similarity: This feature is only considered for smaller regions to merge them easily. If this is not done, the more prominent regions tend to combine with more significant regions, leading to multiple scales in a specific location.

$$
\mathrm{S}_{\text {size }}\left(\mathrm{r}_{\mathrm{i}}, \mathrm{r}_{\mathrm{j}}\right)=1-\left(\operatorname{size}\left(\mathrm{r}_{\mathrm{i}}\right)+\operatorname{size}\left(\mathrm{r}_{\mathrm{j}}\right)\right) / \operatorname{size}(\text { image })
$$

Where size $\left(\mathrm{r}_{\mathrm{i}}\right)$, size $\left(\mathrm{r}_{\mathrm{j}}\right)$ and size(image) are sizes of region and image in pixels respectively.

This entire procedure of selective search is often referred to as Region Proposal. R-CNN is a combination of Region Proposal with CNN. Usually, the CNNs run the sliding windows over the entire image, which is computationally inefficient. However, R-CNN improves the efficiency of computation by using only a few windows. To detect an object with R-CNN:

- Using selective search, generate category independent region proposals and warp them.

- Feed the warped region proposals to a $\mathrm{CNN}$. 
- To extract the features from the CNN, apply $\mathrm{SVM}^{24}$ as it helps classify the objects' presence in the region. Apply Regressor to get the bounding box information.

- For all these scored regions, a non-max suppression algorithm is applied to eliminate the intersection over union (IoU) issue by rejecting the regions with a score higher than the threshold set while learning.

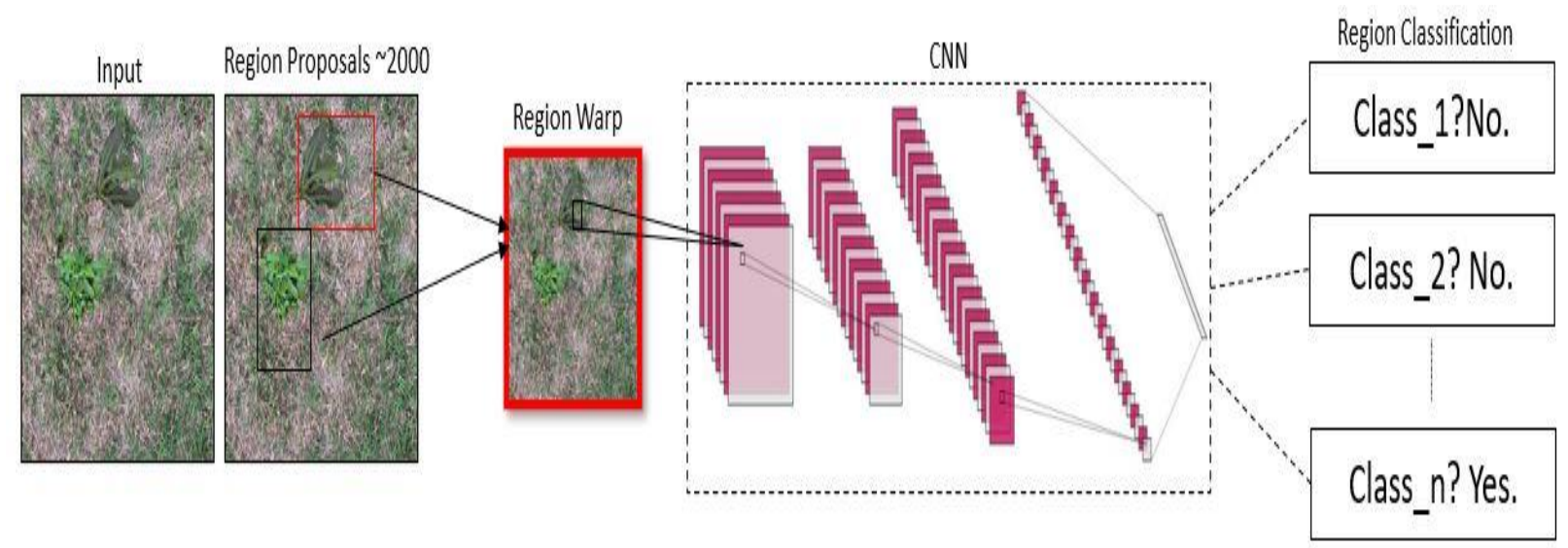

Figure 3.3: Object detection using R-CNN.

To better understand the Non-Max suppression and how it helps eliminate the multiple detections of the same object, we need to understand IoU.

\section{Intersection over Union:}

For a given pair of bounding boxes, one obtained by the ground truth and the other from the algorithm prediction, IoU calculates the intersection over the union of the two bounding boxes as shown in the figure below.

\footnotetext{
${ }^{24}$ Support Vector Machines is a supervised learning algorithm for classification and regression problem.
} 


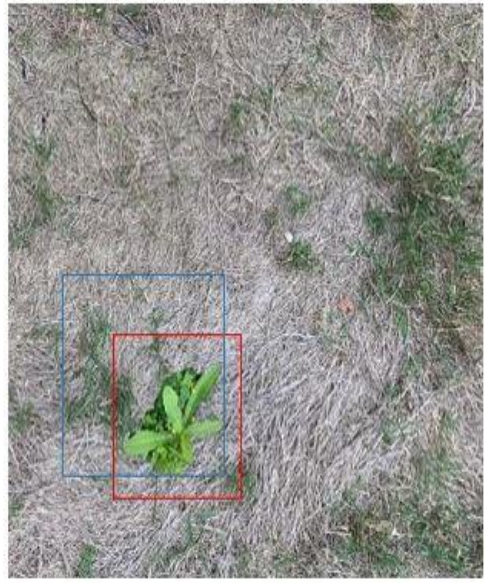

(a)

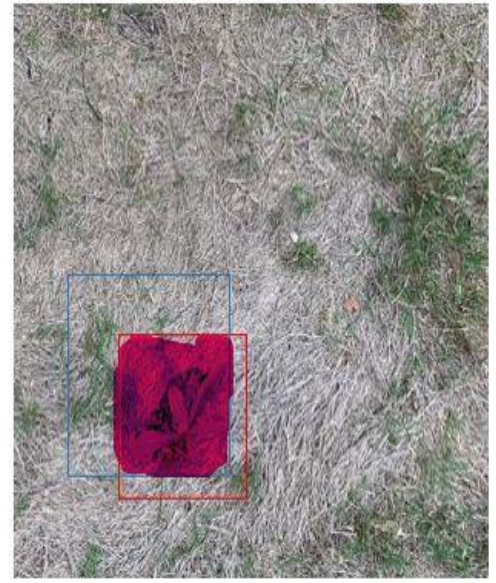

(b)

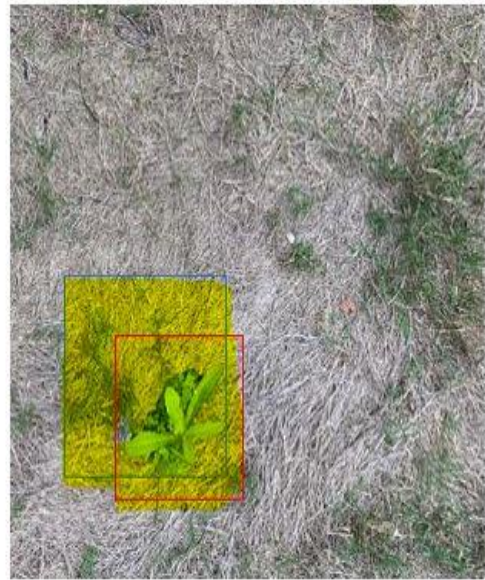

(c)

Figure 3.4: Intersection over Union. (a) Red bounding box is ground truth and the blue bounding box is the predicted bounding box. (b) Intersection of the bounding boxes. (c) Union of the bounding boxes.

Mathematically,

$$
I o U=\frac{\text { Area overlap between the ground truth and predicted bounding box } \epsilon}{\text { Total area of the bounding boxes }}
$$

Non-max suppression usually considers the bounding boxes with $\mathrm{IoU}>0.5$ for object detection, with $\mathrm{IoU}=1$ being that the bounding boxes are overlapping perfectly. If there are multiple bounding boxes with IoU > 0.5, Non- Max suppression usually picks the bounding box with the highest IoU and drops the others for the same object. 


\section{The architecture of R-CNN:}

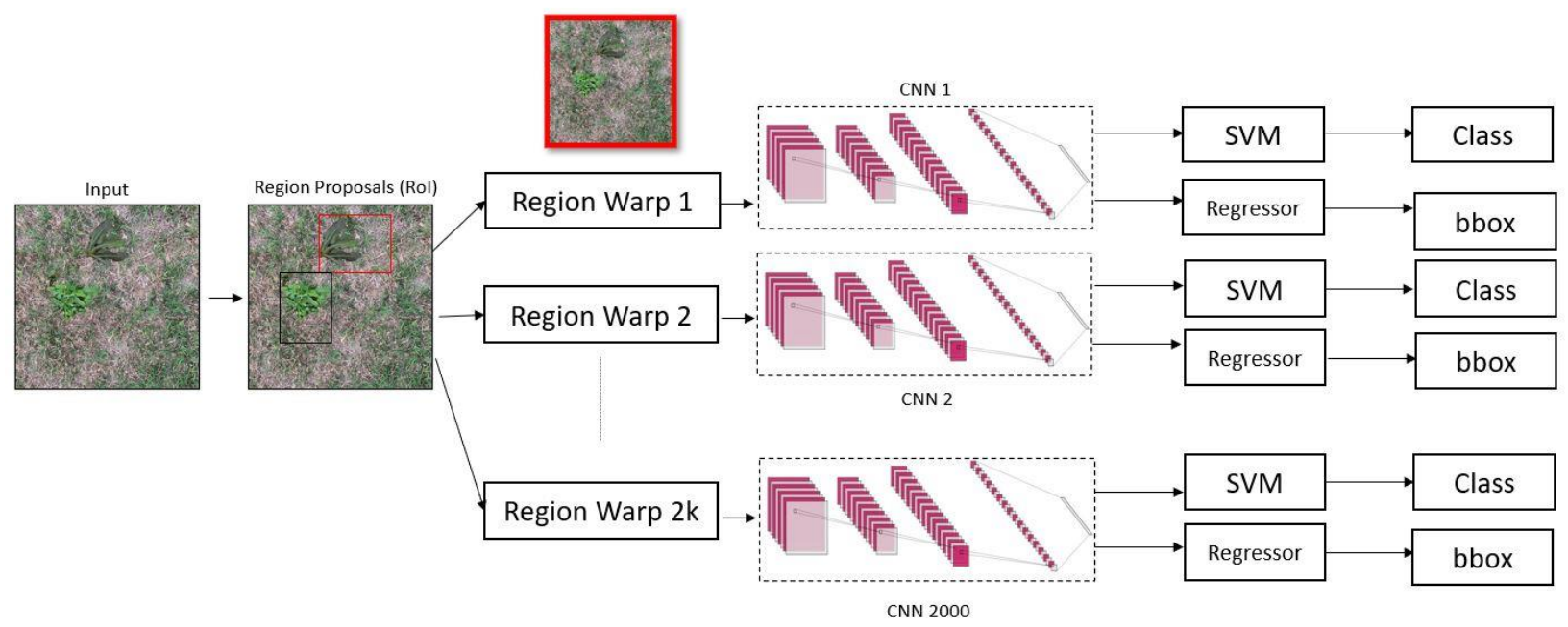

Figure 3.5: The network architecture of $R-C N N$.

The workflow is as follows:

1. The input image is fed to a selective search block to get the 2000 region proposals.

2. These warped region proposals are fed through CNNs to extract the features.

3. The extracted features are sent through SVM for object identification and a Regression block to get the bounding boxes.

\section{Drawbacks of R-CNN:}

Computationally inefficient as we must use 2000 region proposals for each image. So, for X images, the total number of $\mathrm{CNN}$ features would be $X^{*} 2000$. These region proposals make the training slow.

To overcome this, we can use ConvNets instead of 2000 region proposals per image. We can also combine the feature extraction, classifier, and bounding box generator blocks. All of this is done in Fast R-CNN.

\section{Fast R-CNN:}

As the name indicates, Fast R-CNN [40] is a fast framework that uses a CNN to extract the entire image's features instead of 2000 region proposals per image for object recognition in an image. 


\section{The architecture of Fast R-CNN:}

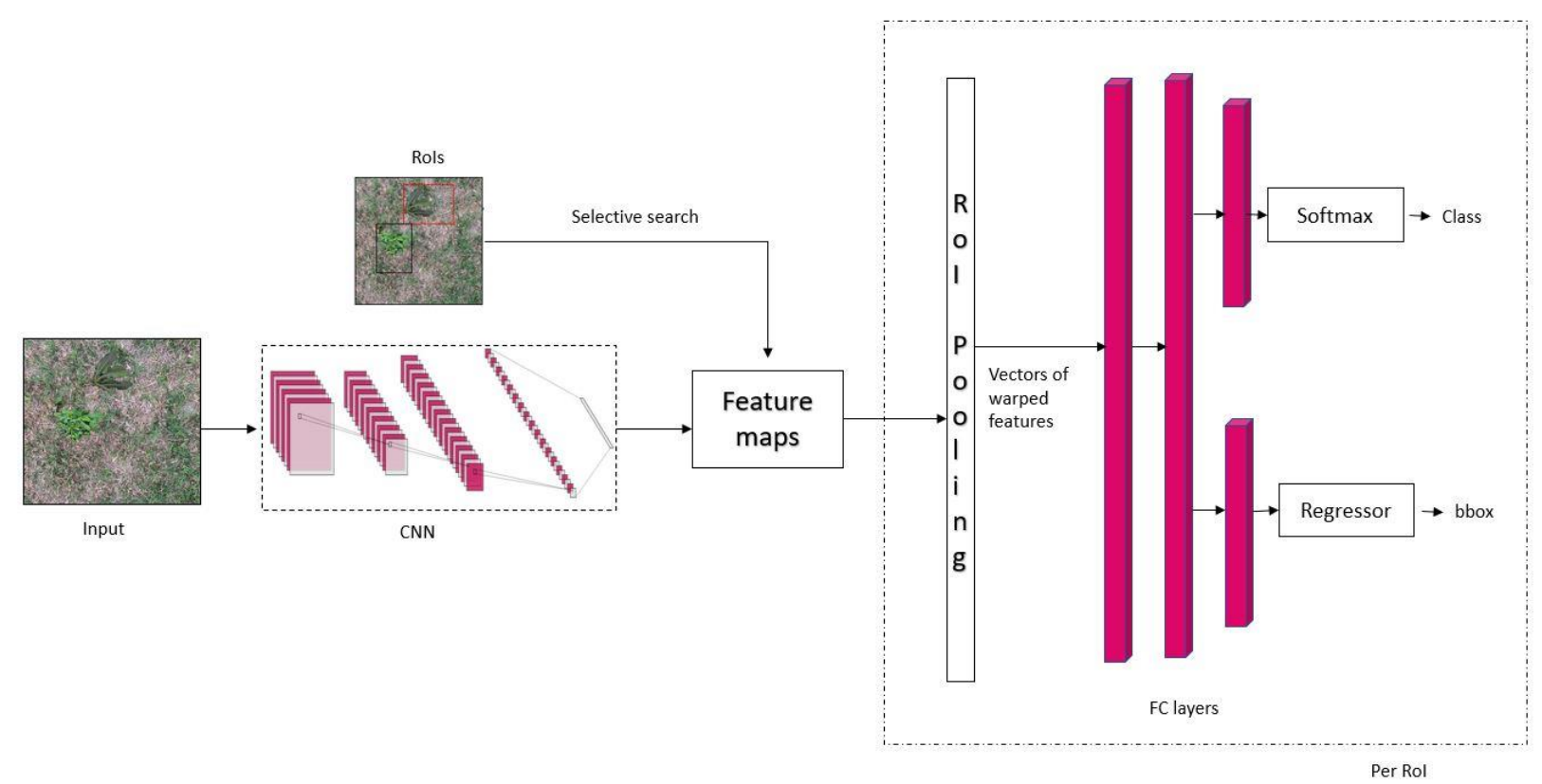

Figure 3.6: The network architecture of Fast R-CNN.

The workflow is as follows:

1. The input image features are extracted using a single CNN.

2. We have an RoI (Region of Interest) pooling block that extracts the fixed-length feature vector from the feature map obtained from the max pooling ${ }^{25}$ layers of the input image.

3. The feature vector obtained from RoI pooling is then fed to Fully Connected (FC) layers of a neural network. However, as the FC network needs a fixed size image as an input, we need to warp the patches of the feature maps extracted from the previous step.

4. The FC network will then perform the localization and classification task.

5. The final output layers of the FC network are Softmax and Regressor. Softmax is a probability block that estimates the object classes and the background, which is a class. Regressor, on the other hand, will give the bounding box information.

\footnotetext{
${ }^{25}$ Finding the maximum value in each feature map's patch
} 
The detection quality is improved because it uses Softmax over SVM as a classifier and the training is single-stage, and training updates all the network layers. Softmax outperforms SVM as it gives the probability for each class while SVM, on the other hand, will be least concerned about the other classes. Hence, SVM is generally helpful if we have a single class classification problem.

\section{Drawbacks of Fast R-CNN:}

Although the performance of Fast R-CNN is improved by using a deep CNN for feature extraction, the selective search for RoI is still expensive computationally when it comes to large real-time datasets. So, the selective search approach for RoI selection can be replaced with another CNN. This limitation gives rise to Faster R-CNN [41].

\section{Faster R-CNN:}

Faster R-CNN's performance is faster than fast R-CNN because it is a single unified network for the object detection problem. It just has a CNN for RoI extraction, referred to as Region Proposal Network (RPN) and the Fast R-CNN model. RPN shares the computation along with the Fast R-CNN block.

\section{The architecture of Faster R-CNN:}

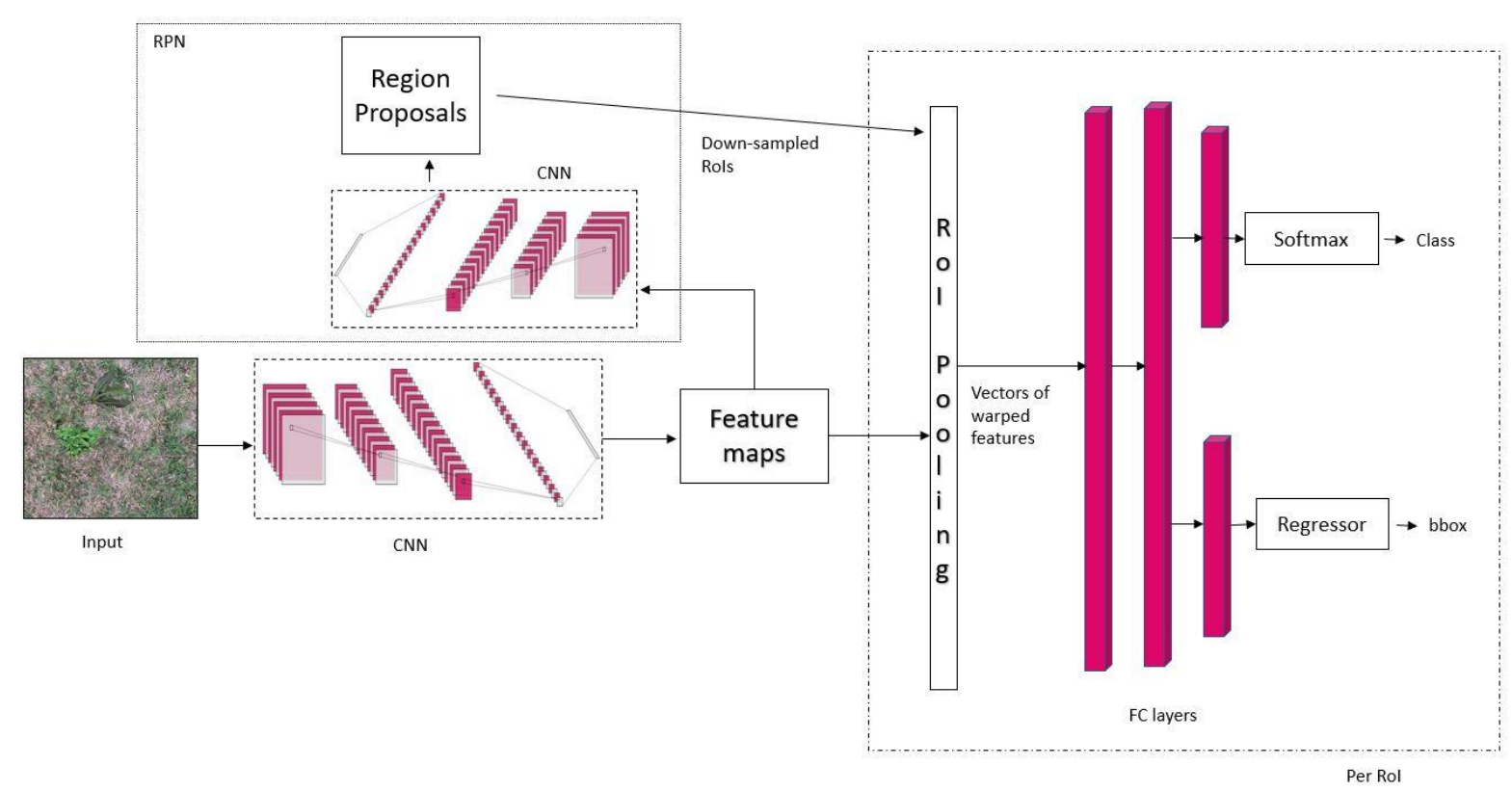

Figure 3.7: The network architecture of Faster R-CNN. 
To understand the Faster R-CNN workflow, we need to understand the Region Proposal Network (RPN) architecture.

\section{RPN:}

RPN is used for the region proposal task in Faster R-CNN. RPN has a unique architecture as it contains regressors and classifiers. The RPN input is an image with an unfixed size, and the output of RPN is a bunch of object proposals, each with an object score. The object proposals are obtained by sliding a little network over the CNN layer's feature map. These object proposals are then fed to two sibling-connected layers: The Classifier - for object identification and the Regressor - for bounding box creation. The architecture of the RPN is shown below.

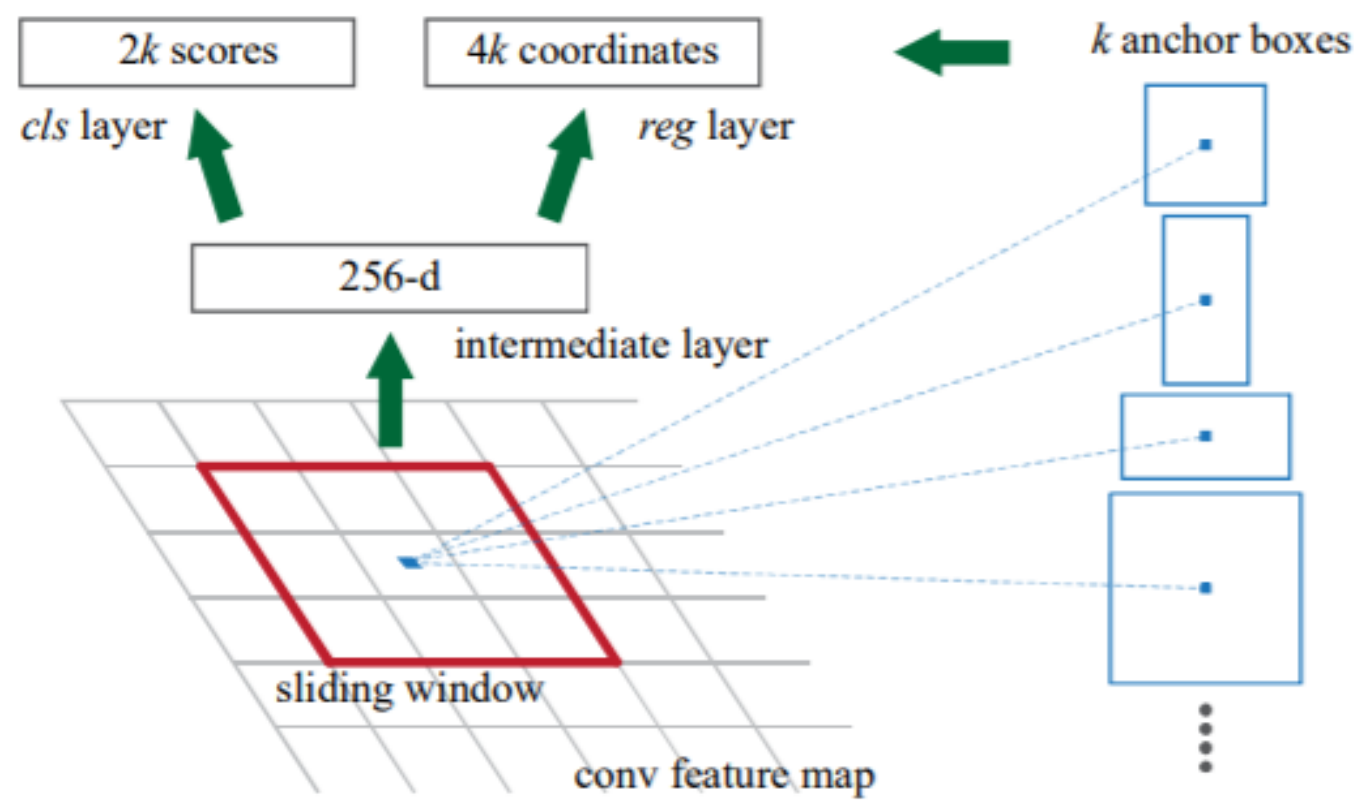

Figure 3.8: Working of RPN. Source [33]

Another critical term that needs to be understood is Anchor. An anchor is placed at the midpoint of the sliding window associated with a scale and aspect ratio. Anchors help with translational invariance that was mentioned earlier. The number of anchors for each sliding window for Faster R-CNN is nine as it uses three scales and three aspect ratios. With these anchors, we can predict multiple region proposals at every sliding window location, with $\mathrm{k}$ being the maximum number of proposals for that location. The object score can be calculated using these $\mathrm{k}$ values. The regression layer outputs 4-k outputs to get the bounding box coordinates, and the classifier layer outputs 2-k 
scores, which will provide the information about the probability of the object present and absent in each region proposal. The number of anchors can be calculated by $\mathrm{W}^{*} \mathrm{H}^{*} \mathrm{k}$, with $\mathrm{W}$ and $\mathrm{H}$ being the width and height of the image. As this algorithm is robust against translations, translation invariance can be achieved. The anchors get their labels based on two factors: anchors with the highest IoU overlapping with ground truth and anchors with $\mathrm{IoU}>0.7$.

All in all, the Faster R-CNN model uses three different neural networks: a Feature Network to extract the feature maps from the given input image, an RPN for getting the RoIs with the highest object containing probability, and finally, a Detection Network for generating the bounding boxes and class of the object. Faster R- CNN can be used for other tasks like image captioning, instance segmentation, 3-D object detection.

To perform instance segmentation, we need to identify each object instance of every known object in an image. So, Faster R-CNN is further extended to Mask R-CNN.

\section{Mask R-CNN:}

Instance segmentation is generally used for counting objects in an image. Instance segmentation usually assigns a label to each pixel in the image. It is more like pixel-level classification. Along with the boundary box creation around the object, we need a segmentation mask around the desired object for the instance segmentation. 


\section{2: Network architecture:}

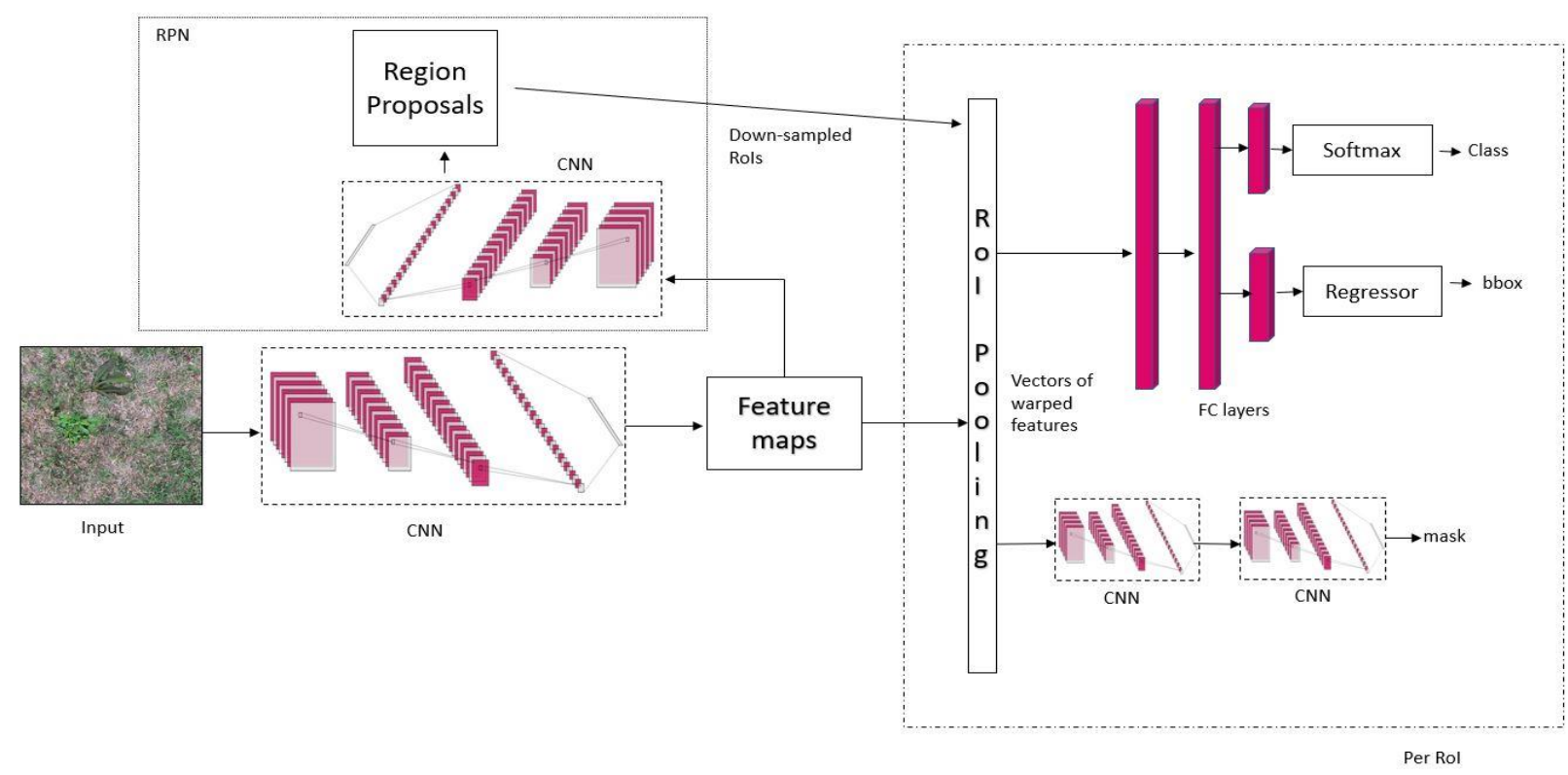

Figure 3.9: The network architecture of Mask R-CNN.

The workflow is as follows:

1. Mask R-CNN has an additional Binary mask classifier block along with the RPN stage as in Faster R-CNN. This block generates masks for every class in the image, and the RPN will give the bounding box details.

2. Every image then goes through a CNN to generate multiple RoI using the mask classifier to obtain the feature maps.

3. The RPN block uses Non-Max suppression to get the nine anchors' highest object scores.

4. Mask R-CNN uses an RoI align network to get multiple bounding boxes around an object and warps it to a single dimension.

5. As in Faster R-CNN, these warped features are fed to an FC network to obtain the object class and the bounding box from Softmax and regressor blocks, respectively.

6. Along with the FC network, the warped features are fed to the Mask Classifier block, which uses multiple CNNs to generate the masks around every class's objects. 


\section{3: Feature extraction with Feature Pyramid Network:}

Detection of objects using multi-scale images for a small object is challenging. We can resolve this problem by using a pyramid of the same image to detect the object, but it is very timeconsuming. We can use this approach when speed is not a requirement. On the other hand, we can use a pyramid of features to extract the objects' information (right-side image). However, this method will not provide accurate object detection.

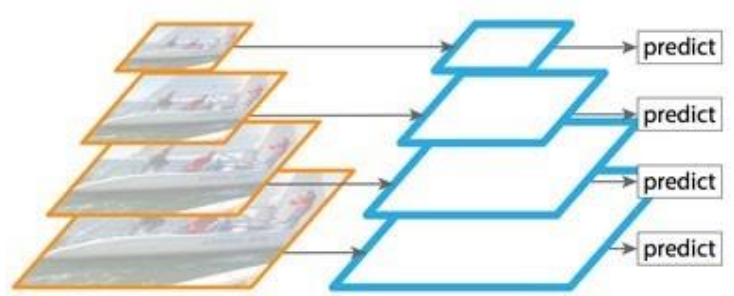

(a)

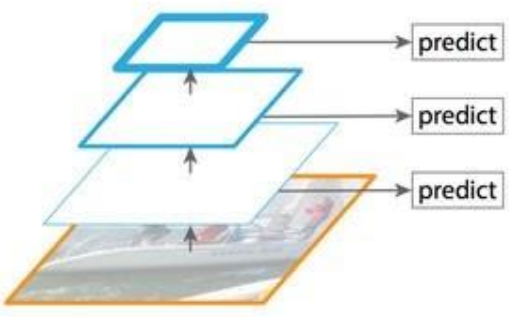

(b)

Figure 3.10: Pyramid architecture. (a) Pyramid of images. (b) Pyramid of feature maps. Source [42]

Feature Pyramid Network (FPN) [42] is designed to address the accuracy and speed limitation issues. FPN can be replaced with the traditional feature extractors to obtain multiple feature maps, which provide better quality feature information. FPN has both bottom-up and top-down pathways. The dataflow looks as follows:

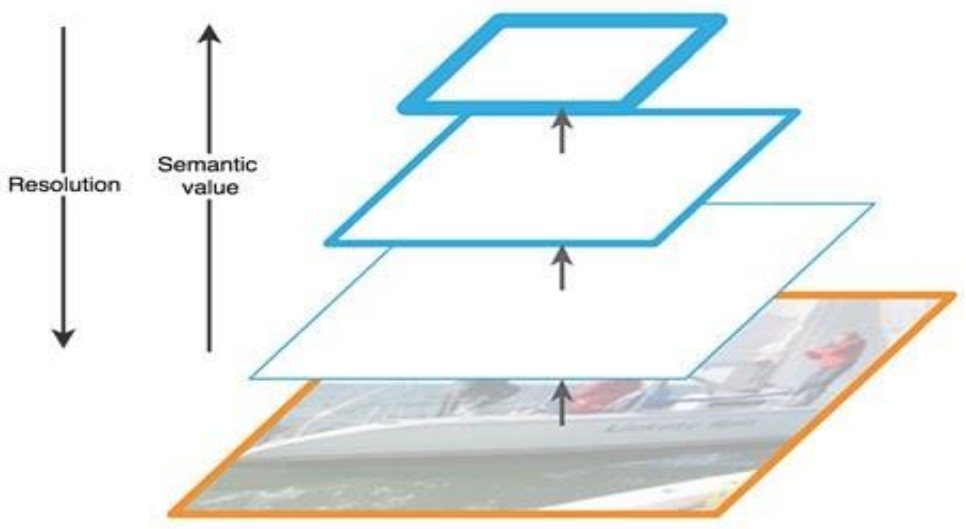

Figure 3.11: Dataflow in an FPN. Source [42] 
As we proceed from the top to bottom layers, the image's spatial resolution increases, and the semantic value decreases. The bottom-up pathway is the standard feature extractors used in CNNs. The top-down pathway is used for Mask R-CNN. It provides a combination of low-resolution semantically robust features with high resolution semantically weak features. FPN grabs all the semantic features from a single scale, therefore, avoiding problems like power consumption and memory.

\section{FPN for RPN:}

Initially, the RPN in Faster R-CNN used a 3x3 sliding window CNN to obtain the bounding box regions and the object classification information. If we replace this conventional technique with FPN, we will no longer be required to have anchors at multi-scales. At each level, multiple aspect ratios are used. Hence, the anchors will be assigned a positive value only if the IoU is $70 \%$. On the other hand, the anchors will have a negative label if the IoU is < 30\%. RoIs of different scales must be fed to the pyramid levels to detect an object in an image.

\section{ResNet 101 Backbone:}

A residual neural network (ResNet) [43] is one of the Artificial Neural Networks (ANNs) that builds on pyramid cells. A conventional ResNet consists of layers of ReLUs aided by batch normalization. Unlike in general CNNs, the residual networks learn residue instead of learning low, mid, and high-level features. Residual of each layer is defined as the subtraction of features learned from the input of that layer. These residual layers help simplify the training process and helps with the degrading accuracy [44] problem because as the depth of the network increases, the accuracy is saturating and starts degrading. There are multiple variants of the residual network, including but not limited to ResNet50, ResNet100, ResNet152. 


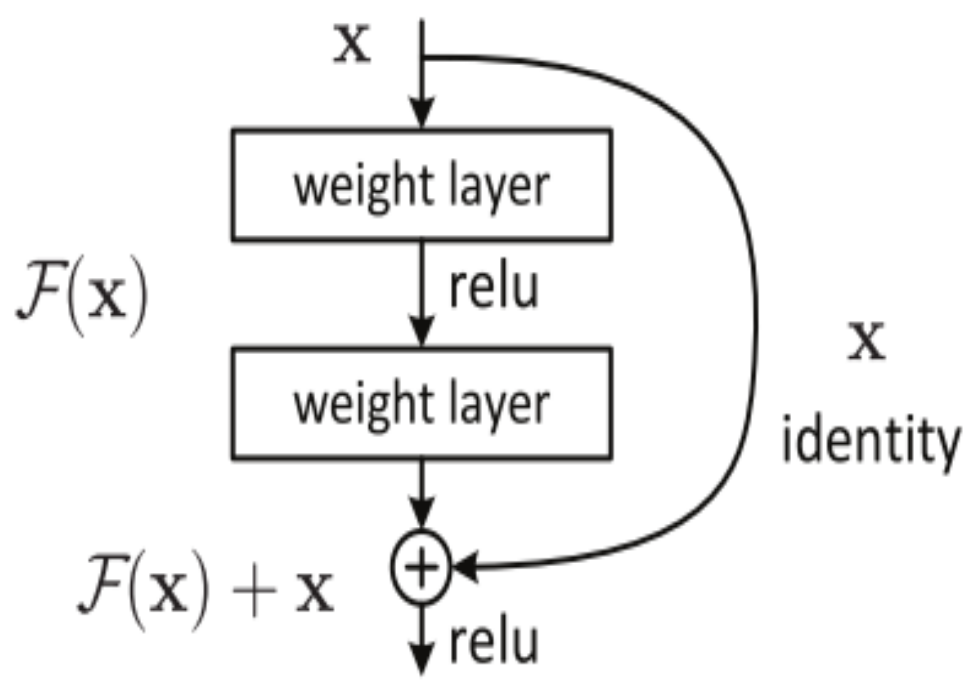

Figure 3.12: Working principle of ResNet. Source [43]

The core idea of ResNet is to skip one or more layers which are often referred to as "identity shortcut connection." Input images are resized, and then a patch of [224 224] is randomly cropped from the image with per-pixel mean subtraction.

\section{4: Dataset:}

One of the challenges for species detection in agriculture is the lack of well-annotated datasets. To use any of the Deep Neural Networks (DNNs), we need to have thousands of images. Unfortunately, there are not very many datasets available online to check the model performance. Hence, we used one of the datasets available online to check Mask R-CNN's performance on the crop vs. weed plants, and we then created a synthetic dataset containing 80 classes. The synthetic dataset is created to expand our study on species analysis as the online dataset has only two to three species of plants.

\subsection{1: CWFID dataset with mask:}

Crop/Weed Field Image Dataset [5] is a dataset created to understand agricultural tasks precision using CV. CWFID dataset is a relatively small dataset as it has only 60 images, and these images are field images collected from an organic carrot field. Along with the RGB images of the 
size $640 \times 480$, the vegetation masks were also provided. These masks help to create an annotation. The 60 images were split into 48 training images and 12 testing/validation images to maintain the split ratio of 4:1. To train the Mask R-CNN, we need to have more than 60 images to get better results. So, we have used a data augmentation technique that is explained in section 4.3. We have generated 2064 training images and 516 testing images. The images below are an example of the RGB image and the annotated mask.

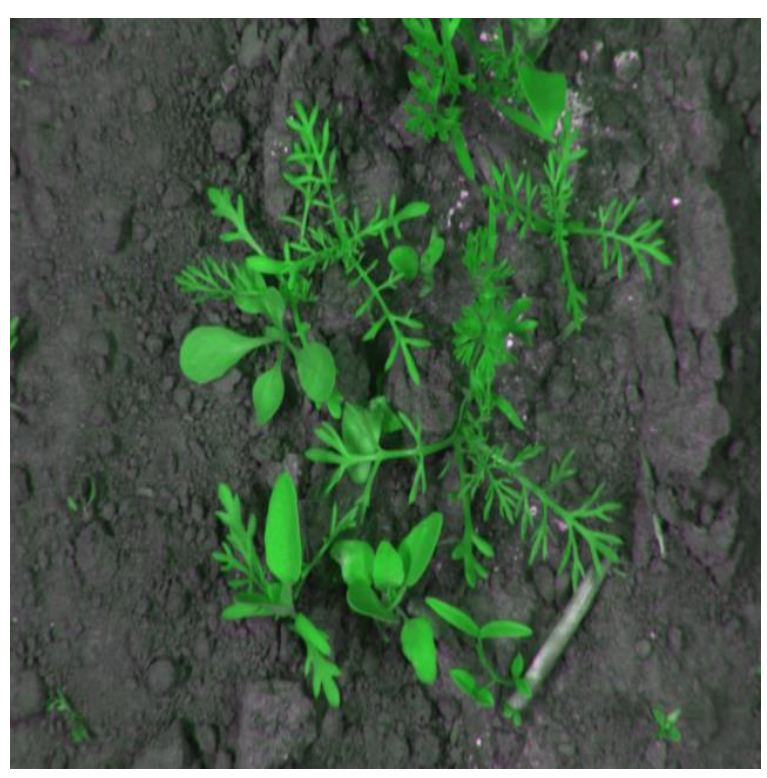

(a)

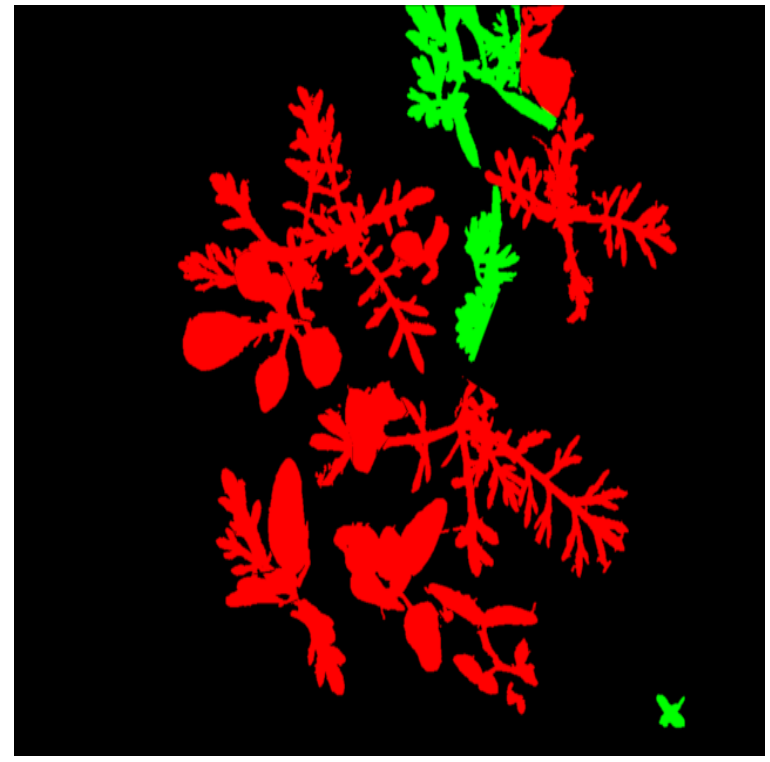

(b)

Figure 3.13: Images from CWFID. (a) RGB image of weed and crop plants. (b) Annotated image with the red colour being the weed plant and green colour being the carrot crop plant. Source [5]

The image on the right side is the annotated mask with the red colour as the weed plant and the green colour as the crop plant. In this section, we will be using Mask R-CNN with MS COCO [32] weights. Hence, we converted this small dataset into a coco format, meaning creating the JSON file for all annotations.

The annotation part provides the information about the bounding boxes from the segmented mask. The iscrowd field determines the nature of the object, with iscrowd $=0$ being an individual object and iscrowd $=1$ being a crowd like a crowd of people in a stadium. The category id field will provide the information of the category and the super-category details. Segmentation is usually Polygon, with $\mathrm{x}$, y being the top-left point and width and height being the bounding box's 
size. Run-Length Encoding or RLE is used to separate the foreground and background in an image and is generally used if iscrowd $=1$.

We have generated the bounding boxes for our COCO type CWFID dataset by using the predefined mask definitions. The masks are defined based on the red and green colours. The red colour $(255,0,0)$ is considered a weed plant, and the green colour $(0,255,0)$ is the crop plant. As there are only two classes, the super-category is fixed to Plant.
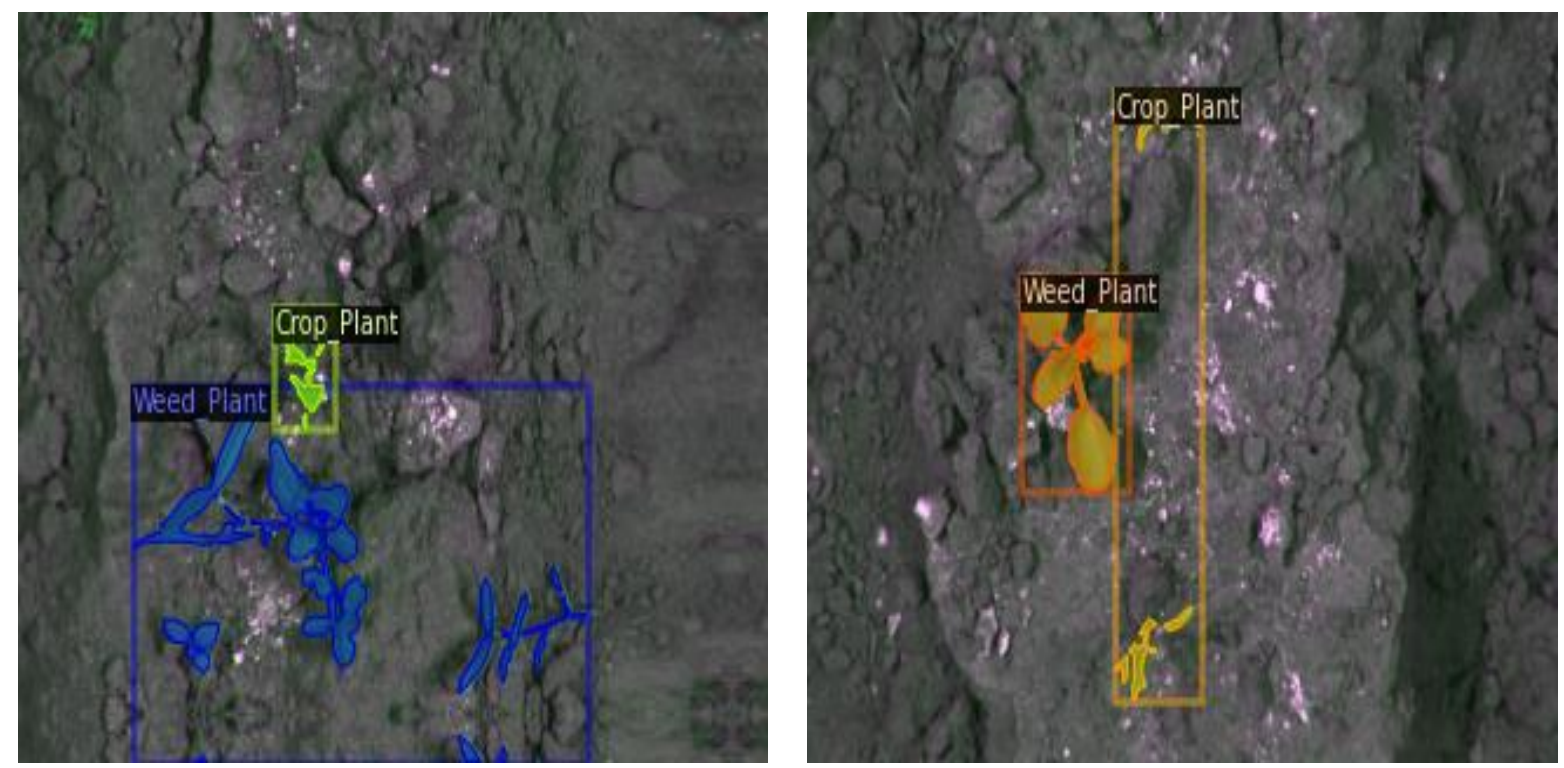

Figure 3.14: Example images from the COCO type CWFID dataset.

\section{Limitations:}

This dataset's drawbacks are that it considers all the weed plants as a single class, and the crop plants also fall under one species, to be precise, organic carrot. To test the performance of Mask R-CNN for multiple classes, we created a synthetic dataset with 80 classes in it.

\subsection{2: Synthetic COCO dataset:}

In this dataset, we created images with multiple plants in them. We have used 80 foreground images of weed plants ${ }^{26}$ and a few background images as shown below:

\footnotetext{
${ }^{26}$ https://www.immersivelimit.com/course/creating-coco-datasets.
} 

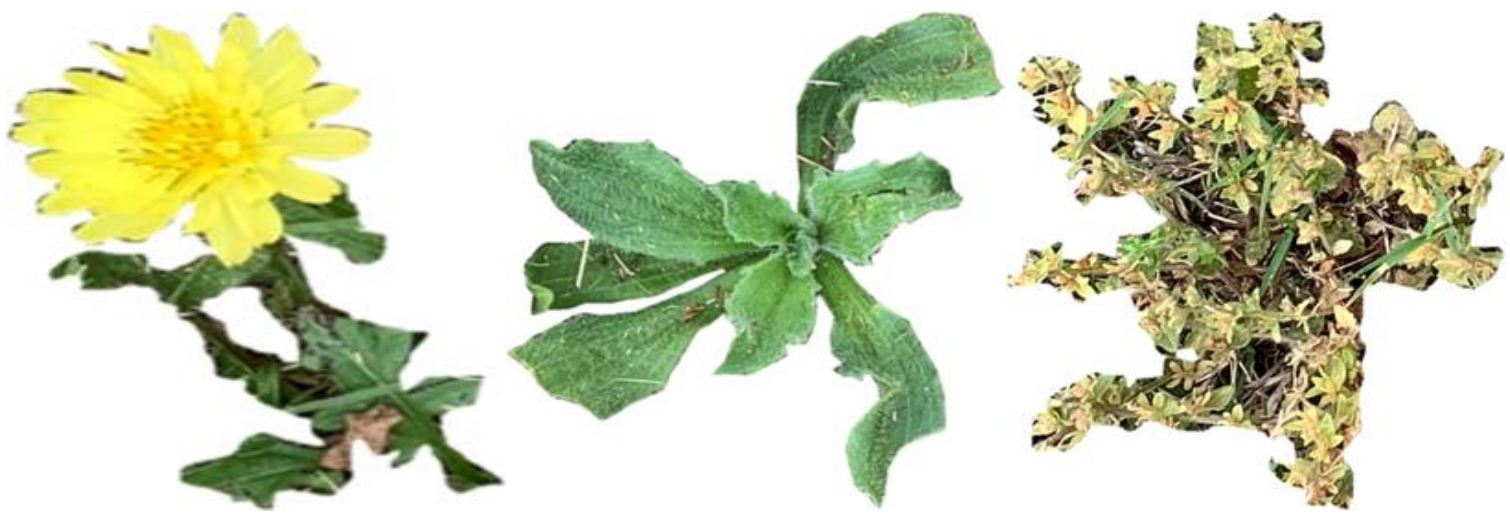

(a)
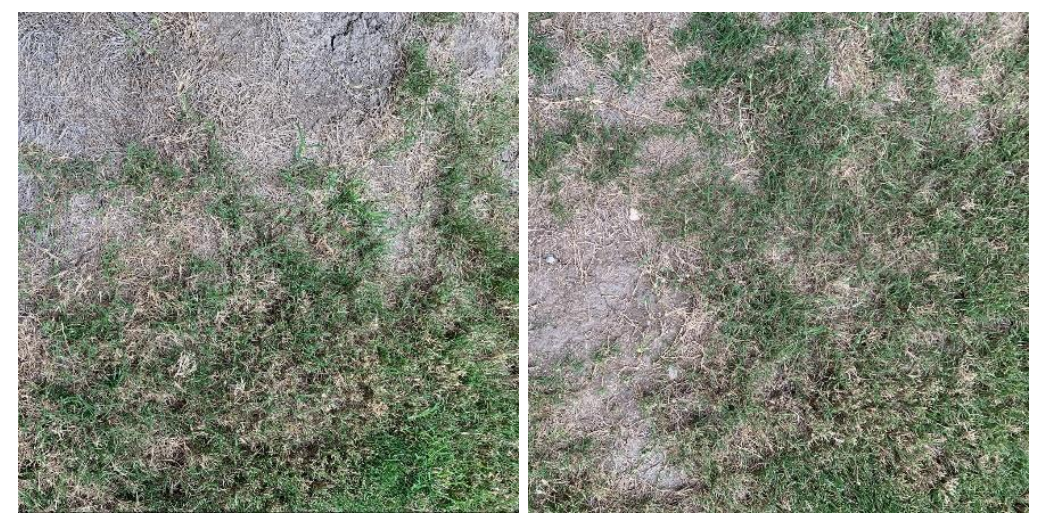

(b)

Figure 3.15: Types of images used for creating the synthetic data with 80 classes. (a) Foreground examples. (b) Background examples.

As we are currently unaware of these species' names, we named our plants class_1 to class_80. Using random placements, we have placed these foreground images on the background images. The masks were created using the alpha layer of the foreground images. We have generated the COCO JSON file using the masks to get the details about the bounding boxes. The iscrowd is set to 0 , and the super-category is set as weeds.

We have generated 10000 training images and 2500 testing images. Although the number can be increased, we set it low just to check if the model can detect the classes or not. With the masks and the annotations, the synthetic images look like the following: 


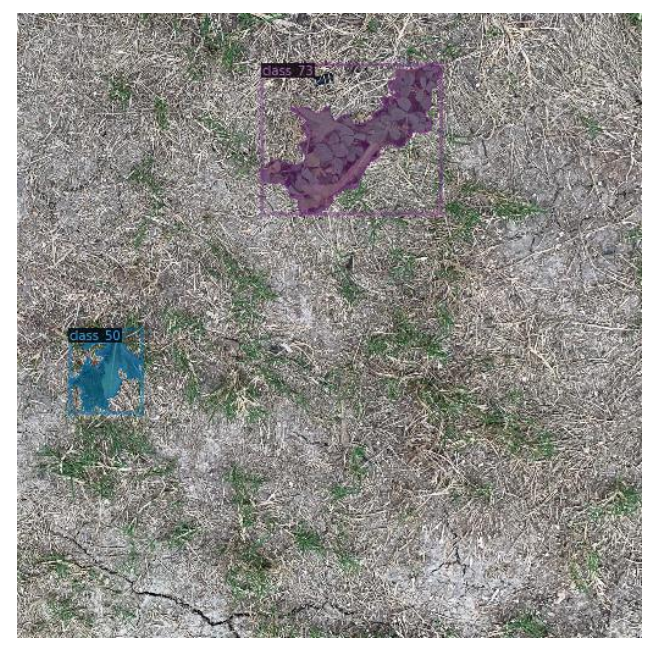

Figure 3.16: Example image from the COCO type Synthetic dataset.

\section{Dataset Comparison:}

\begin{tabular}{|c|c|c|c|c|c|c|}
\hline Dataset & $\begin{array}{c}\text { Number of } \\
\text { Images }\end{array}$ & $\begin{array}{c}\text { Number of } \\
\text { Classes }\end{array}$ & Task & Channel & Size & Labels \\
\hline $\begin{array}{c}\text { Plant - } \\
\text { Seedling }\end{array}$ & 960 & 12 & Segmentation & RGB & Variable & 960 \\
\hline $\begin{array}{c}\text { Agriculture- } \\
\text { Vision [6] }\end{array}$ & 96,984 & 6 & $\begin{array}{l}\text { Segmentation } \\
\text { (aerial) }\end{array}$ & RGB, NIR & $512 \times 512$ & 168,086 \\
\hline $\begin{array}{c}\text { Dataset of } \\
\text { Tomato leaves } \\
{[9]}\end{array}$ & 14,531 & 10 & Classification & RGB & $227 \times 227$ & 14,531 \\
\hline $\begin{array}{c}100 \text { leaves } \\
\text { plant species } \\
{[8]}\end{array}$ & 1,000 & 16 & Classification & Binary & variable & 1,600 \\
\hline $\begin{array}{c}\text { DeepWeeds } \\
{[12]}\end{array}$ & 17,509 & 8 & Classification & RGB & $256 \times 256$ & 17,509 \\
\hline CWFID [5] & 60 & 2 & Segmentation & RGB & $640 \times 480$ & 494 \\
\hline $\begin{array}{c}\text { Our Synthetic } \\
\text { data }\end{array}$ & 12,500 & 80 & Segmentation & RGB & $1000 \times 1000$ & 19,963 \\
\hline
\end{tabular}

Table 1: Statistical comparison between other datasets and our synthetic dataset 


\section{5: Average Precision:}

In this thesis, we evaluate Mask R-CNN's performance on various datasets by using Average Precision (AP). In general, precision is defined as

$$
\text { Precision }=\frac{\text { True Positive }}{\text { True Positive }+ \text { False Positive }}=\frac{\text { True Positive }}{\text { Total detections }}
$$

Where,

True Positive $=$ A correct prediction of a positive class

False Positive $=$ An incorrect prediction of a positive class

False Negative $=$ An incorrect prediction of negative class

True Negative $=$ A correct prediction of a negative class

For $\mathrm{COCO}$ datasets or COCO type datasets, mean average precision (mAP) is often referred to as Average Precision. To better understand AP, we need Recall along with Precision. A recall is defined as

$$
\text { Recall }=\frac{\text { True Positive }}{\text { True Positive }+ \text { False Negative }}=\frac{\text { True Positive }}{\text { Total Ground truths }}
$$

$A P$ is given by calculating the area under the Precision-Recall curve.

$$
A P_{T}=\int_{0}^{1} P(r) d r
$$

Where,

$\mathrm{P}(\mathrm{r})=$ Precision as a function of Recall

$\mathrm{T}=\mathrm{IoU}$ threshold (For AP50, $\mathrm{T}=50 \%$ and for AP75, $\mathrm{T}=70$ ) 

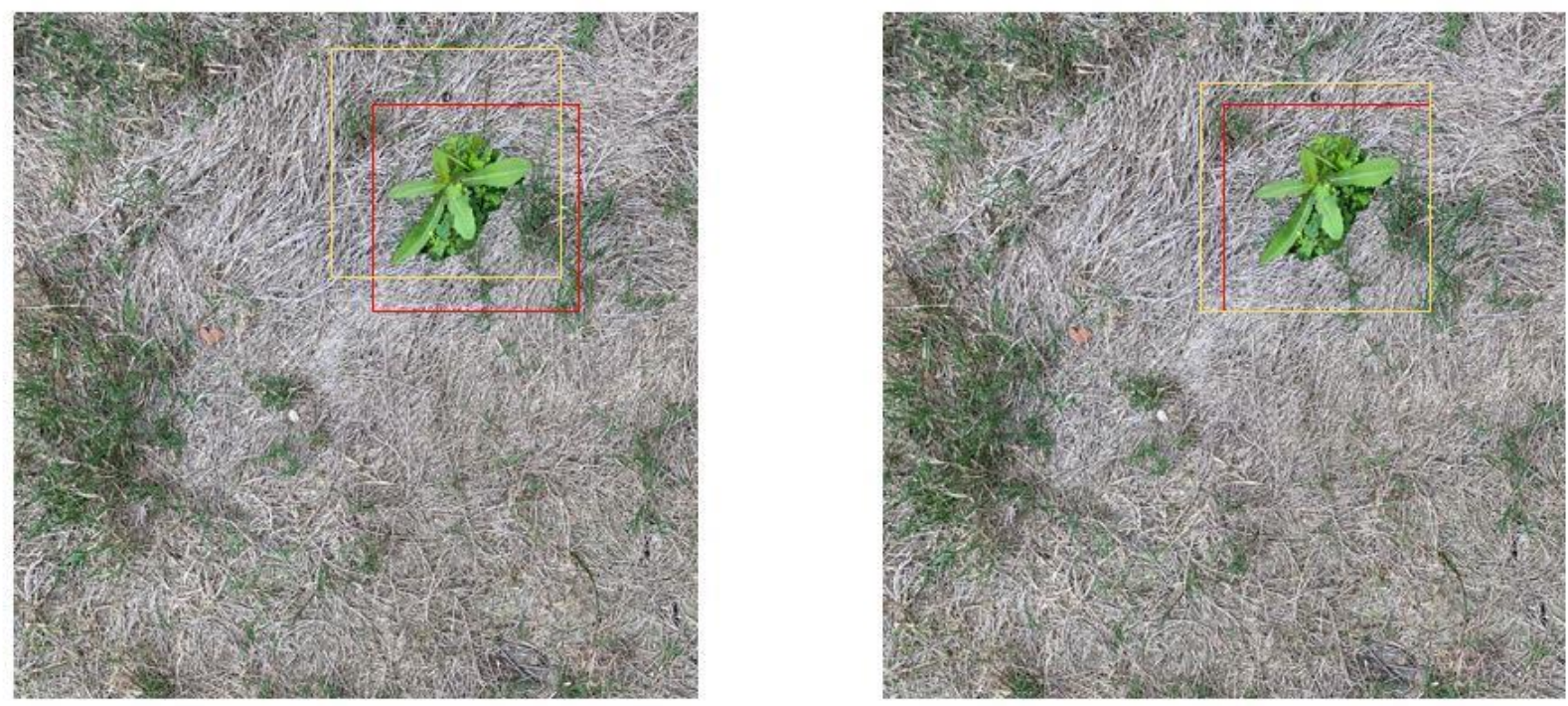

Figure 3.17: $I o U=0.5($ left $), \mathrm{Io} U=0.75$ (right)

AP for a class,

$$
A P[\text { class }]=\frac{1}{\text { Different Thresholds }} \sum A P(\text { class }, I o U)
$$

AP for all classes,

$$
A P=\frac{1}{\text { All Classes }} \sum A P(\text { class })
$$

Therefore, AP50 = AP at IoU @ 0.5 and AP75 = AP at IoU @ 0.75.

\section{6: Experiment setup:}

\subsubsection{Using CWFID:}

Using the Mask R-CNN model to detect the crop vs. weed plants, we have used Resnet 50 FPN as a backbone for feature extraction. The learning rate used is 0.001 , and the number of epochs is 1000. The batch size is 4 , and the number of classes is 3 (crop, weed, and background). We used Detectron2 to conduct this experiment. 


\subsubsection{Using Synthetic COCO dataset:}

The experiment setup is the same with some slight modifications. The batch size is 2 , and the number of classes would be 81 ( 80 weed classes and one background class).

\section{7: Results and Discussion:}

CWFID: As the dataset is good enough for 2 class information, we have set the Softmax threshold to 0.9 . The results are as follows:
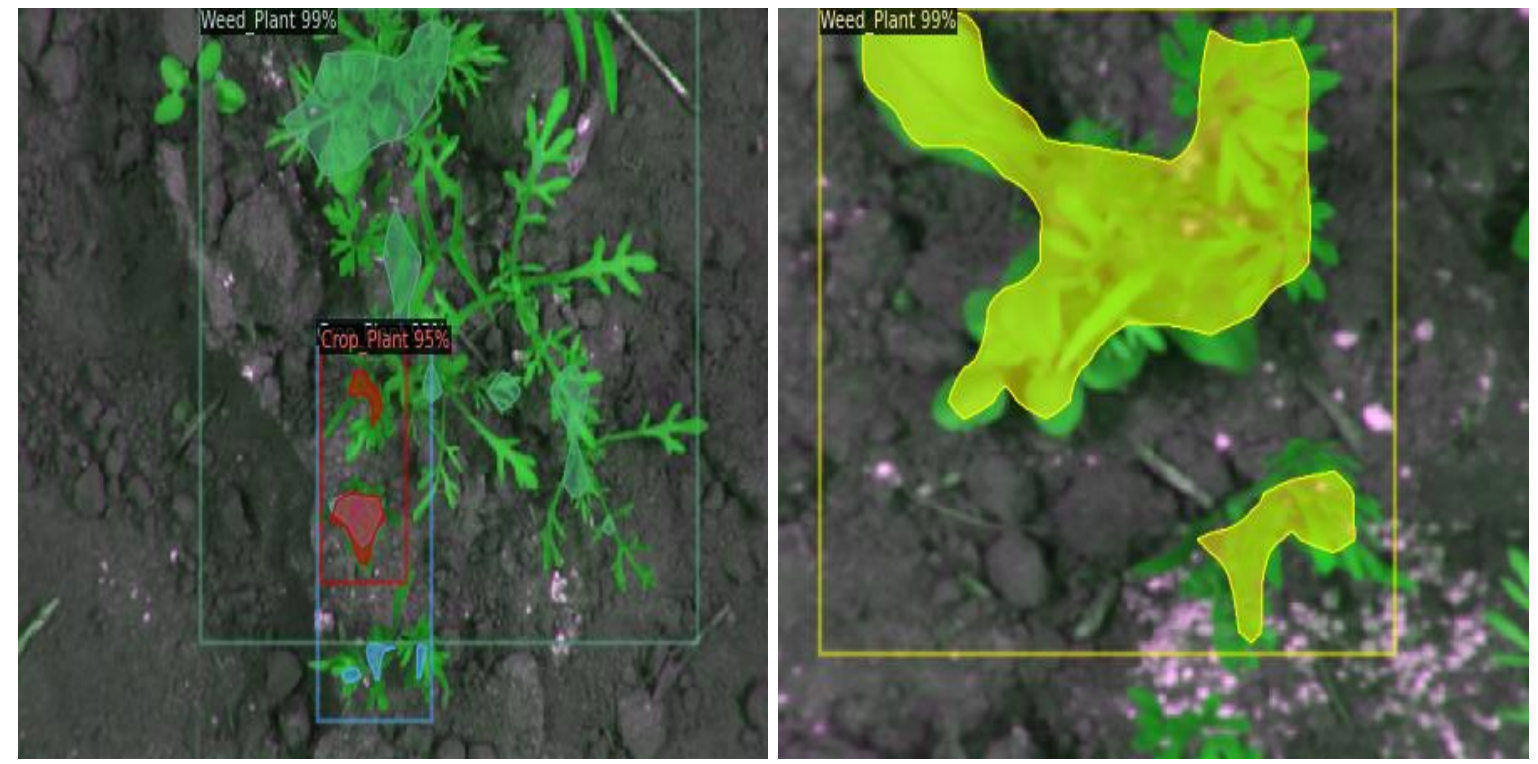

Figure 3.18: COCO type CWFID's Mask R-CNN results with Softmax threshold $=0.9$. The number on the top of the bounding box is the probability of the plant being a specific class like Crop_Plant or Weed_Plant.

Synthetic COCO dataset: The dataset created is minimal for an 80-classes classifier. The original COCO dataset has 2,500,000 labelled images for 80 classes. The comparison of performance is not possible due to a lack of enough information with our dataset. Ideally, 1000 images per class are preferred. We have set the Softmax threshold to 0.5. 

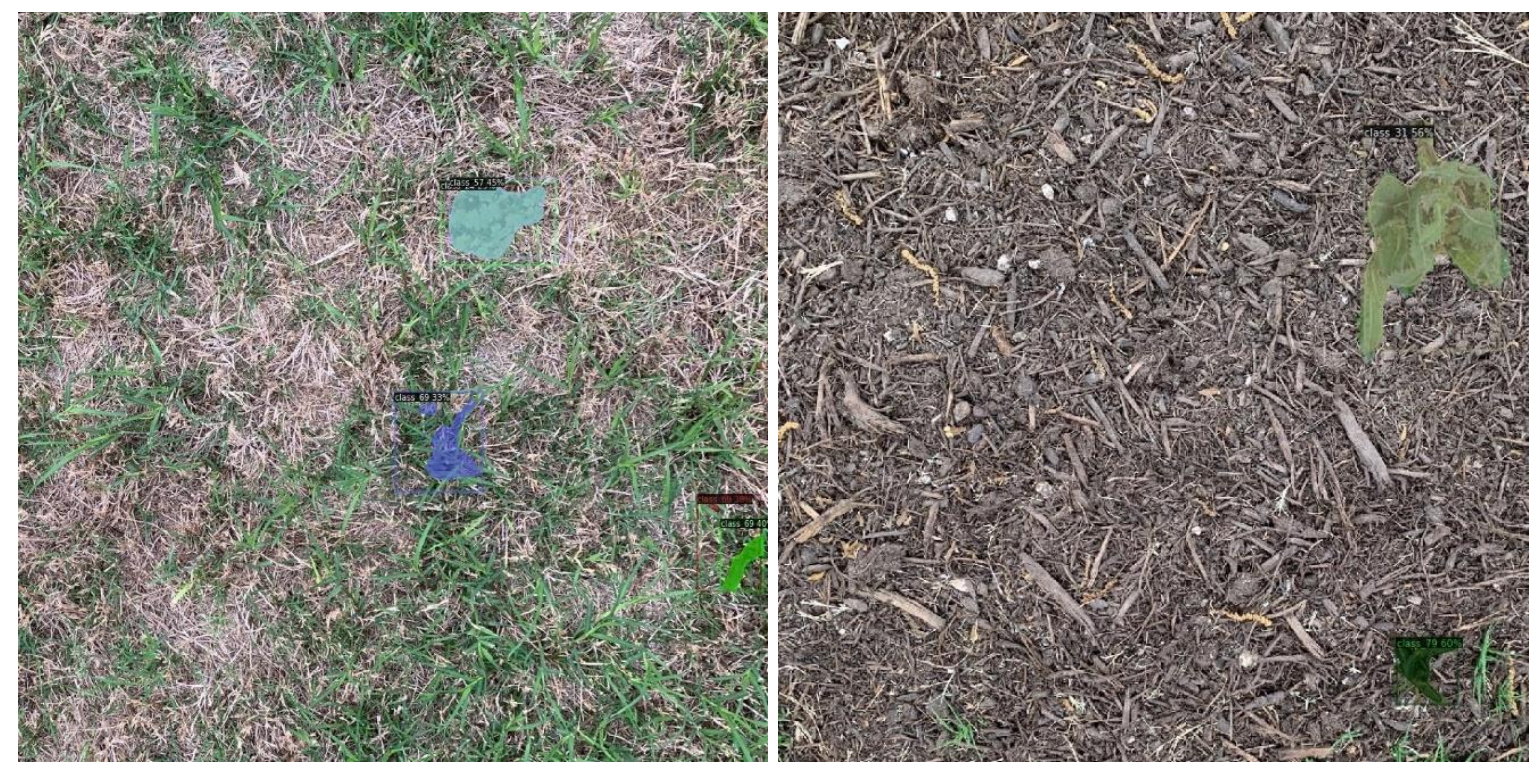

Figure 3.19: Synthetic COCO dataset's Mask R-CNN results with Softmax threshold $=0.5$. The number on the top of the bounding box is the probability of the plant being a specific class like Class_1-Class_80.

\section{Discussion:}

To analyse Mask R-CNN's performance, we adjusted the synthetic dataset to 2 classes, one crop plant and the rest as weed plants, and reduced the number of images to 1250. This dataset is still an advancement over CWFID as CWFID has only two types of weeds, and we have $\sim 75$ weed types in our weed class.

\begin{tabular}{|c|c|c|c|c|}
\hline Dataset & \# images & \# Classes & AP & AP50 \\
\hline CWFID [5] & 60 & 2 & 79.6 & NA \\
\hline Adjusted Synthetic data & 1250 & 2 & 50.03 & 75.952 \\
\hline
\end{tabular}

Table 2: Comparison of Mask R-CNN's performance on ground-level plant recognition image with CWFID.

Our adjusted synthetic data was able to reach 50\% accuracy. Nevertheless, it can be improved with more crop species and thereby avoiding the data imbalance ${ }^{28}$ problem.

As we do not have enough information about the weed plant species, our 80-class dataset might even have redundant species in them. Despite all these limitations, Mask R-CNN was able to

\footnotetext{
${ }^{27}$ Average precision at $\mathrm{IoU}=0.5$

${ }^{28}$ Image distribution among the classes is skewed (biased).
} 
localize the plants on a given image. To progress our study on MAM (see chapter 5), we need to have sufficient data to test it with Mask R-CNN. 


\section{Chapter 4:}

\section{Data Augmentation for Aerial Images:}

\subsection{Introduction:}

When we consider the most popular datasets, the number of images ranges from thousands or tens of thousands or even millions. For getting an improved performance of the CNN, we need to have large datasets. Data Augmentation is a technique used to increase the number of data points or images in our case by adding the slightly altered copies of available limited data or creating new synthetic data as mentioned in section 3.4.2. By doing this data augmentation, we can reduce the problem of overfitting while training a neural network. When we train a DNN or any other machine learning model, we are tuning the model's parameters to map an image input to a label output with a goal to reduce the model loss. The current State-of-the-art (SOTA) CNNs have millions of parameters. It is only logical to have a proportionate number of images to obtain decent performance. As most object recognition or localization algorithms exhibit translational invariance (section 3.1), this property will serve as a baseline for the data augmentation. Data augmentation can be as simple as flipping the images, changing the image's orientation, or changing its scale or brightness. Data augmentation will be helpful even if we have a large amount of data as well. For instance, in the synthetic data created in section 3.4.2, if all of the class_1 species are left-aligned and class_2 species are right-aligned, the model is likely to get confused when we provide class_1 species that is right-aligned, although if the model has a $90 \%$ accuracy. It is because the model will learn that all left-aligned will most likely be class_1. Hence, if we use data augmentation, this 
problem can be solved. There are multiple options to achieve this data augmentation. Some of them are:

1. Neural Style Transfer

2. Geometrical transformations

\subsection{Style Transfer:}

\subsubsection{Introduction:}

Usage of Neural Style Transfer is an advanced technique for data augmentation as it focuses on the colour, the texture of the image and addresses illumination variation problems, unlike conventional data augmentation techniques such as flipping, cropping, and rotation. NST will be a good choice if we consider the semantic content of the image with translational invariance. As we need thousands of images for our weed detection, we created a dataset using the localized style transfer technique.

\subsubsection{Localized Style Transfer:}

As mentioned in section 2.2.2, we have used localized style transfer to transfer the Mile-AMinute style from the images we have collected to some aerial images obtained from the Agriculture - Vision 2020 dataset [6]. These images are taken from various farmlands across the United States to address semantic segmentation in agriculture. These images are of exceptional quality, and they are well annotated. Compared to other agriculture datasets, this dataset has a relatively high number of images in RGB and $\mathrm{NIR}^{29}$ channels. As most object recognition datasets focus on everyday objects, it is challenging to understand agriculture images' patterns without proper datasets. As we focus on MAM recognition using UAVs, we need more images to train our neural networks effectively. To address this issue, we used a localized style transfer technique using CNN [45]. In general, the content loss function and style loss functions play a crucial role in

\footnotetext{
${ }^{29}$ Near Infra-Red
} 
NST. We adjust the style loss function in the localized style transfer technique to apply the style to masked regions. In this thesis, we used VGG19 to achieve our goal of creating synthetic data.

\subsubsection{VGG19 network Architecture:}

As the name suggests, VGG19 has 19 layers to extract the features from a given image. This network has been trained on the ImageNet dataset [46]. The shallow layers of this network focus on the pixel-level information while the deep layers, on the other hand, focus on the object localization. VGG19 is a variant of VGG developed by Visual Geometry Group. Some of the other available variants of VGG are VGG11, VGG16, VGG19. The VGG architecture outperformed many SOTA models for ImageNet Large-Scale Visual Recognition Challenge (ILSVRC). As ImageNet is a classifier dataset, in simple terms, VGG is an image classifier CNN. The architecture of VGG19 is as follows.

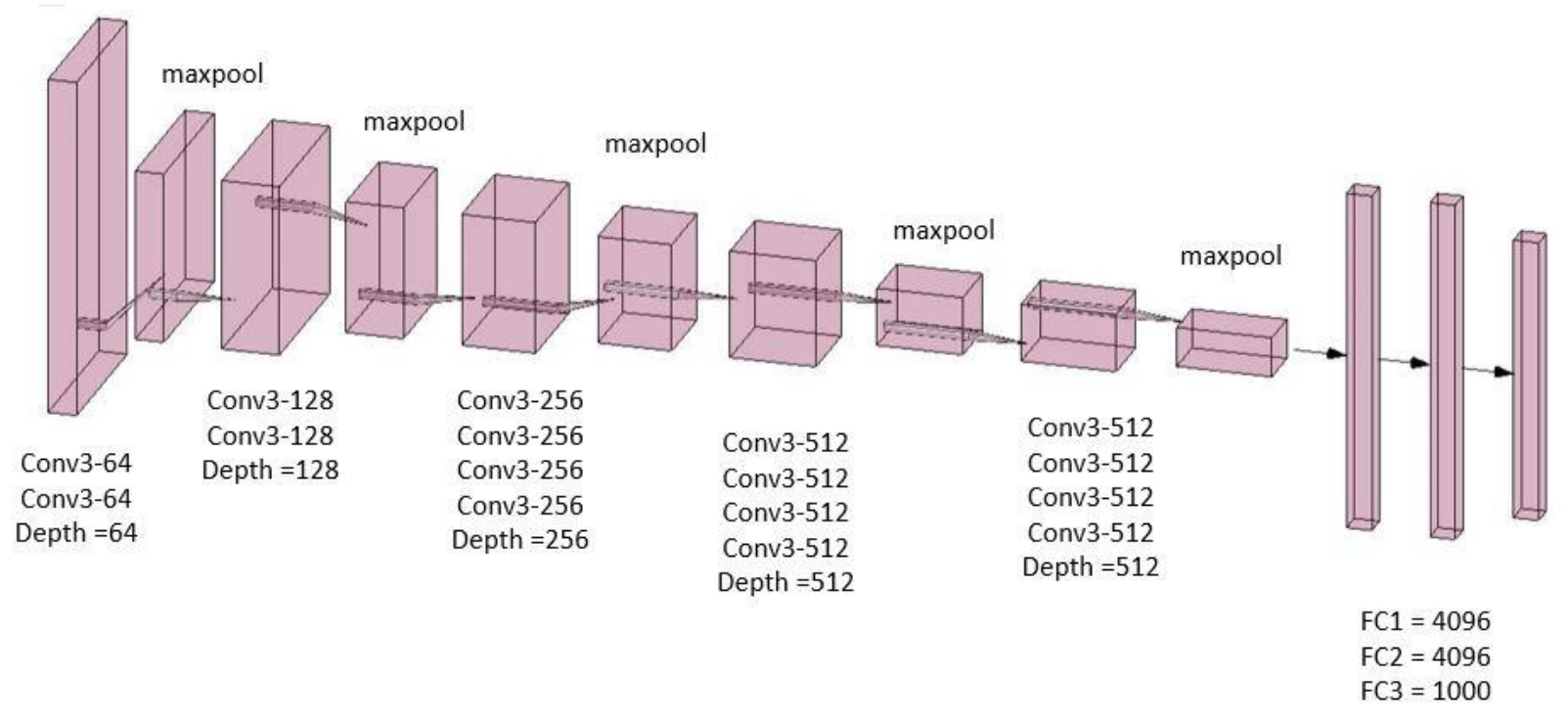

Figure 4.1: The network architecture of VGG19.

The workflow is as follows:

1. An RGB image of size $224 \times 224$ is given as an input to the network. The input image size is fixed, thereby leading to a fixed shape of $(224,224,3)$.

2. The mean RGB value is subtracted from every pixel as pre-processing.

3. A kernel of size $3 \times 3$ is used to slide over the entire image with a stride of 1 . 
4. Spatial padding is done to preserve the spatial resolution of the input image.

5. Down-sampling is done by using max pooling with a window size of $2 \times 2$ and stride 2 .

6. ReLu units are introduced to improve computational efficiency and classification efficiency. As discussed in section 3.1, ReLu is a better activation function choice than sigmoid functions or hyperbolic tangents.

7. The images are then fed to three FC layers. As shown in the below table, the first two FCs have a size of 4096, and the third FC has a size of 1000. The ReLu layers are not mentioned in the table below to avoid complexity.

8. The final layer of VGG19 is a Softmax function to provide the probability of the classification.

\section{ConvNet Configuration of VGG19}

19 weight layers

224x224 Input Image (RGB)

Conv3-64

Conv3-64

Max pooling

Conv3-128

Conv3-128

Max pooling

Conv3-256

Conv3-256

Conv3-256

Conv3-256

Max pooling 


\begin{tabular}{|c|}
\hline Conv3-512 \\
Conv3-512 \\
Conv3-512 \\
Conv3-512 \\
\hline Max pooling \\
\hline Conv3-512 \\
Conv3-512 \\
Conv3-512 \\
Conv3-512 \\
\hline Max pooling \\
\hline FC-4096 \\
\hline FC-4096 \\
\hline FC-1000 \\
\hline Softmax \\
\hline
\end{tabular}

Table 3: ConvNet configuration of VGG19

Although the VGG architectures are designed for image classification challenges, they can be used in several other applications. For instance, we will be using this architecture for style transfer by finding the content and style loss of an image.

\subsubsection{VGG19 for style transfer:}

Before we discuss the functionality of VGG19 for NST, we will look at the primary usage of a CNN in NST. The encoding feature of CNN will play a crucial role in NST. The basic working of a CNN based NST is as follows: 


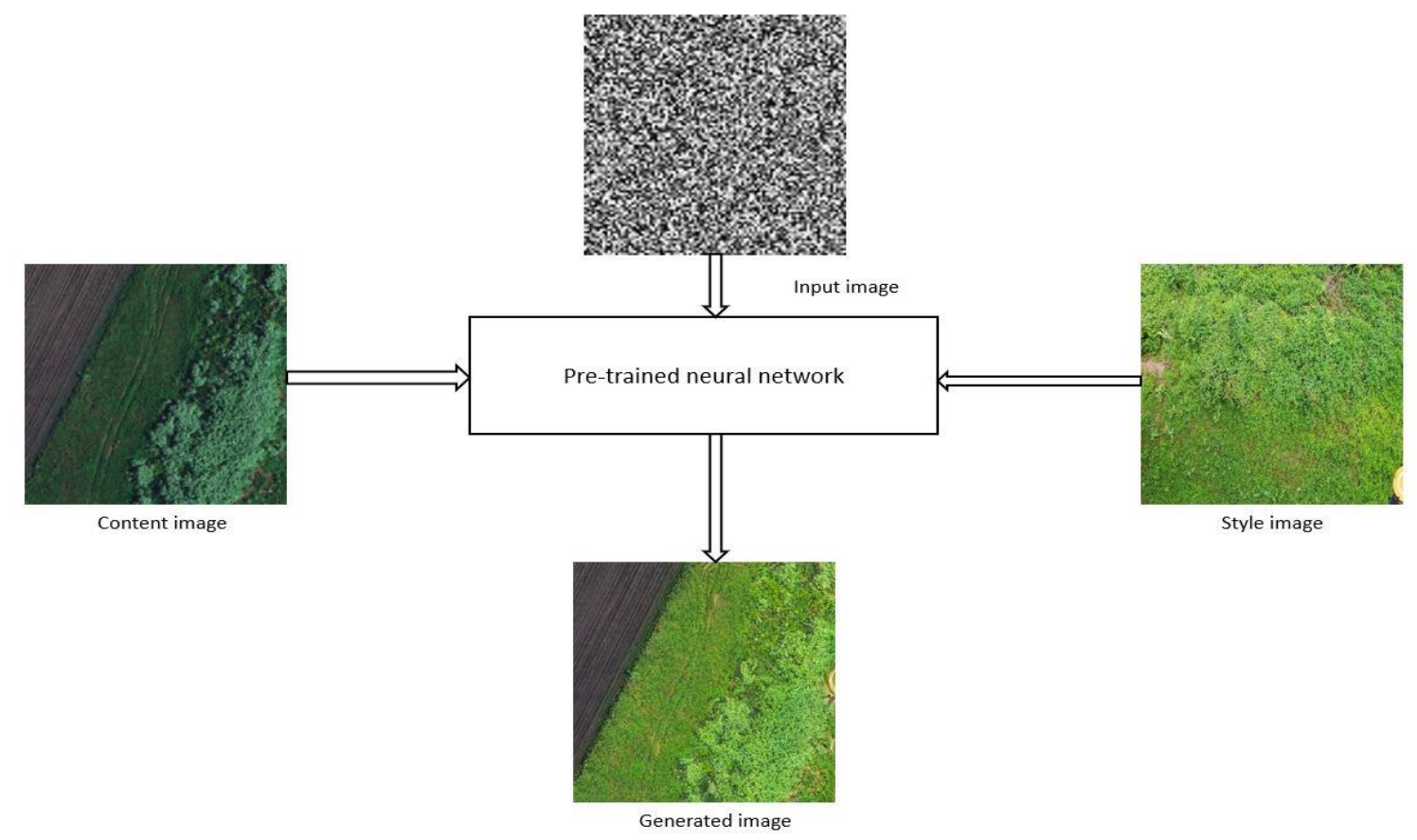

Figure 4.2: Concept of NST with CNN.

We have a noisy input image fed to the CNN to serve as our output image. Along with the input image, we have a content image (C), and a style image (S) fed to CNN. Our objective is to find how identical is our input image to the content and style images, respectively, at every layer in the VGG. We need to preserve the content of the content image and the style of the style image. So, we define and calculate the content and style loss as follows:

\section{Content loss:}

This measure gives us the similarity quotient of our noisy input image with the content image at a given layer (L) in VGG. If $\mathrm{P}$ and $\mathrm{F}$ are the original and generated images, $\mathrm{F}(1)$ and $\mathrm{P}(1)$ are their L layer values. The content loss is given as:

$$
\mathrm{L}_{\text {content }}=\frac{1}{2} \sum_{i, j}\left(F_{i j}^{l}-P_{i j}^{l}\right)^{2}
$$

\section{Style Loss:}

To understand the style loss, we need to have a better understanding of the term style. The style information is calculated by using the correlation value of the feature maps in a layer. The loss function is obtained by taking the difference between feature maps produced by the style image and the generated image. 


$$
\begin{gathered}
\mathrm{L}_{\text {style }}=\sum_{l} w^{l} L_{\text {style }}^{l} \\
\mathrm{~L}_{\text {style }}{ }^{1}=\frac{1}{M^{l}} \sum_{i, j}\left(G_{i j}^{l}(s)-G_{i j}^{l}(g)\right)^{2} \\
G_{i j}^{l}(I)=\sum_{k} A_{i k}^{l}(I) A_{j k}^{l}(I)
\end{gathered}
$$

Here, $\mathrm{w}^{1}$ is the weight of the layer L. $\mathrm{G}$ is a Gram matrix ${ }^{30}$ often referred to as a style matrix. The figure below explains how the Gram matrix values are calculated.

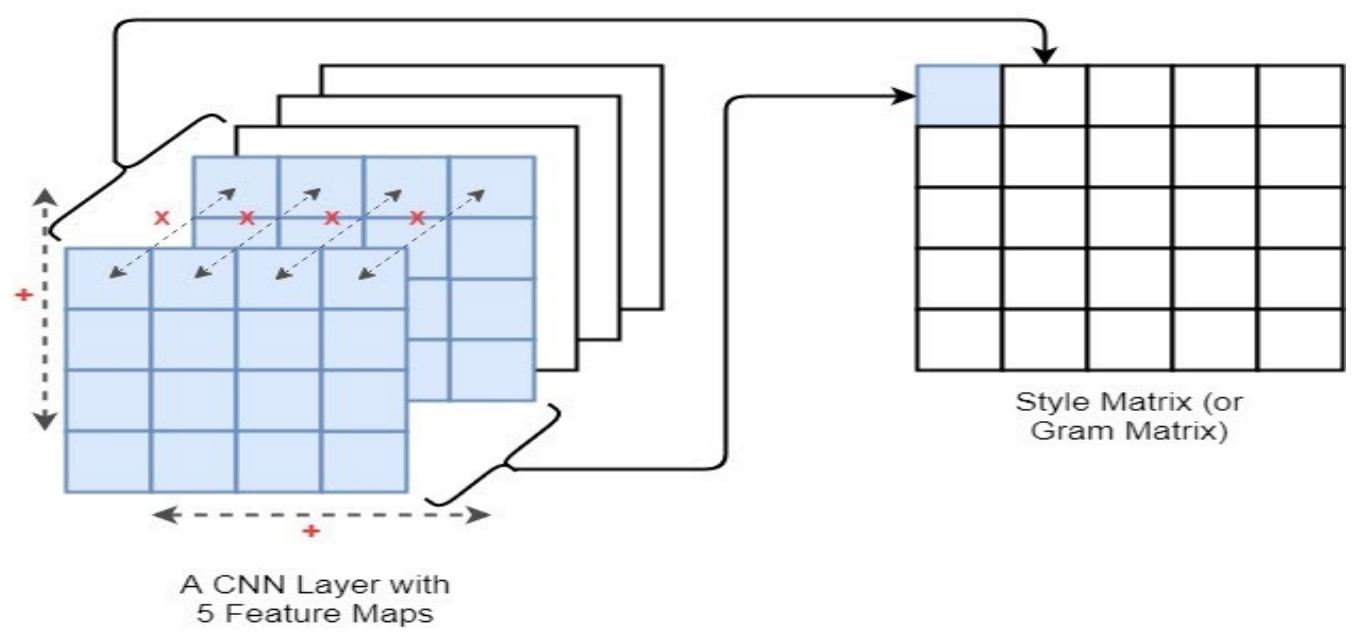

Figure 4.3: Computation of Style Matrix with 5 feature maps in a CNN layer. Source ${ }^{31}$

For every $\mathrm{G}_{\mathrm{ij}}$, the $\mathrm{i}^{\text {th }}$ and $\mathrm{j}^{\text {th }}$ feature maps are multiplied, and then they are summed in both width and height dimensions. Here $\mathrm{A}$ is the feature map. $M$ is a hyperparameter that varies with the layer. By trying to reduce the style loss, we try to match the two images' feature distribution.

The final loss is obtained by summing both content and style loss.

$$
L=\alpha L_{\text {content }}+\beta L_{\text {style }}
$$

\footnotetext{
${ }^{30}$ https://mathworld.wolfram.com/GramMatrix.html.

${ }^{31} \mathrm{https} / / / \mathrm{www}$.analyticsvidhya.com/blog/2020/10/introduction-and-implementation-to-neural-style-transferdeep-learning/.
} 
Here, $\alpha$ and $\beta$ are user-defined parameters to adjust the amount of content and style loss that needs to be injected into the final generated image.

There are multiple options for us to use this NST. We can transfer the style without colour, texture transfer to the entire image, or segmented image. We have used the last option. Here are the examples of each case.

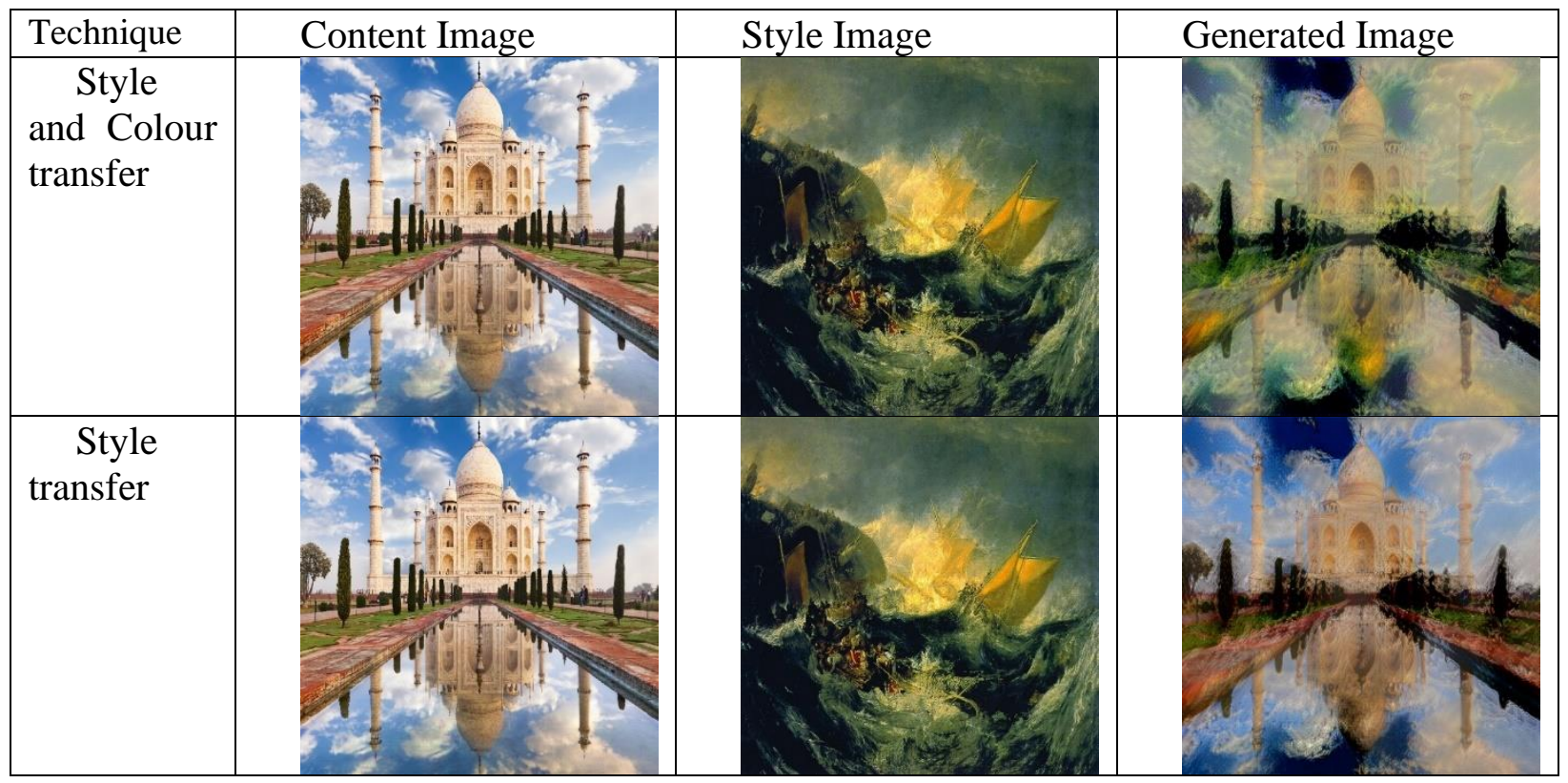

Table 4: Illustration of Global style transfer with and without colour transfer from the style image to the content image. 


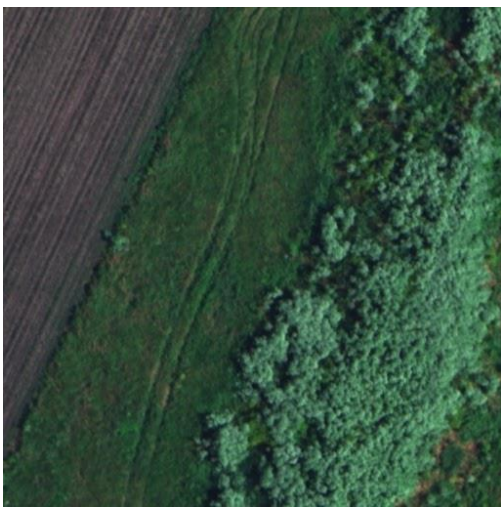

(a)

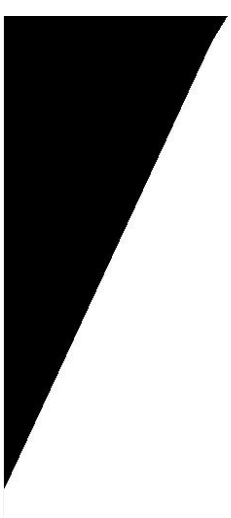

(b)

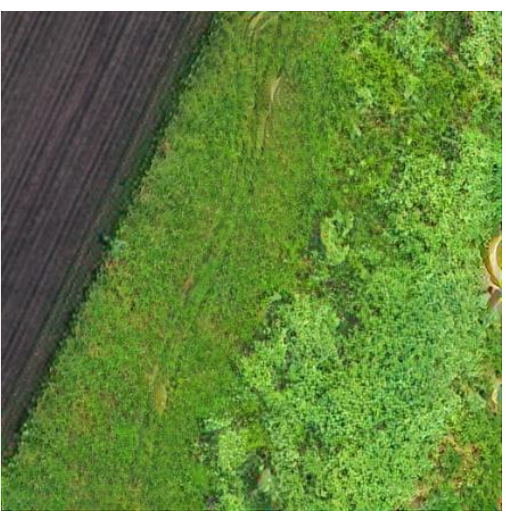

(c)

Figure 4.4: An example of localized style transfer. (a) content image, (b) Mask of the content image, (c) Generated image with a random style. Source [6]

As we want to perform the style transfer with semantic segmentation, we need to modify the style loss function to transfer only to the RoI.

Algorithm for semantic segmentation style transfer [47] :

1. Take patches from the semantic layer or the mask for both style and generated images.

2. Find the nearest neighbour for a given patch in a layer by finding the cross-correlation.

$$
N N(i)=\arg _{j} \min \frac{\Psi_{s}(s) \cdot \Psi_{j}\left(s_{s}\right)}{\left|\Psi_{s}(s)\right| \cdot\left|\Psi_{j}\left(s_{S}\right)\right|}
$$

3. The style error between all the patches and the generated image in that particular layer can be calculated by finding the Euclidean distances' summation [Reference].

$$
E_{S}\left(s, s_{s}\right)=\sum_{i}\left\|\Psi_{s}(s)-\Psi_{N N(i)}\left(s_{s}\right)\right\|^{2}
$$

\subsubsection{Hyperparameters:}

The hyperparameters $\alpha$ and $\beta$ helps to determine the amount of style that needs to be transferred with a minimum loss. The content weight $(\alpha)$ value chosen for our dataset creation is $5 \mathrm{e} 0$, and the 
style weight $(\beta)$ value is $1 \mathrm{e} 4$. These values are chosen based on visual experimentation. However, we can change these parameters accordingly based on the user requirements.

\subsubsection{Dataset:}

We used the Agriculture-Vision dataset as our content images and our UAV images of MAM (Chapter 5) as style images to obtain the style transfer. The Agriculture-Vision dataset comes with a binary mask which we used for our semantic segmentation part.

- Aerial images from Agriculture-Vision: This dataset has 94,986 images each of size $512 \times 512$. The main aim of this dataset is to identify the visual patterns in agriculture. It contains images in both RGB and NIR channels. The 94,986 images include RGB, NIR, and segmented masks.

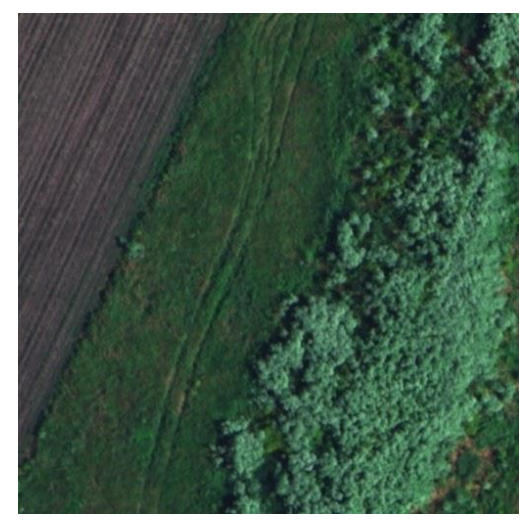

(a)

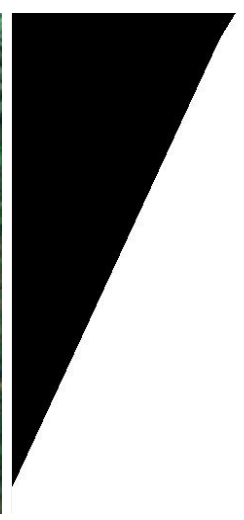

(b)

Figure 4.5: Sample images of Agriculture-Vision dataset's weed cluster class. (a) RGB image of the weed cluster, (b) Binary mask for the RoI. Source [6]

This dataset contains six different image classes, including cloud shadow, double plant, planter skip, standing water, waterway, and weed cluster. As we are interested in weed clusters, we have just used the weed cluster class images. However, the other classes can be used if we need a classifier model.

- MAM Aerial Images: We have obtained a few real-time drone images of MAM at the height of 5-15 meters above the ground surface. Using a human expert's help, we were able 
to get MAM boundaries for 15 images. The image size is $5472 \times 3648$ as these images are taken using a high-quality camera. Since the image size is huge, we need to resize it to avoid crashing the deep learning model and incorporate the 512 x 512 Agriculture-Vision dataset. The red boundaries shown below are human-made, and they provide information about the MAM.
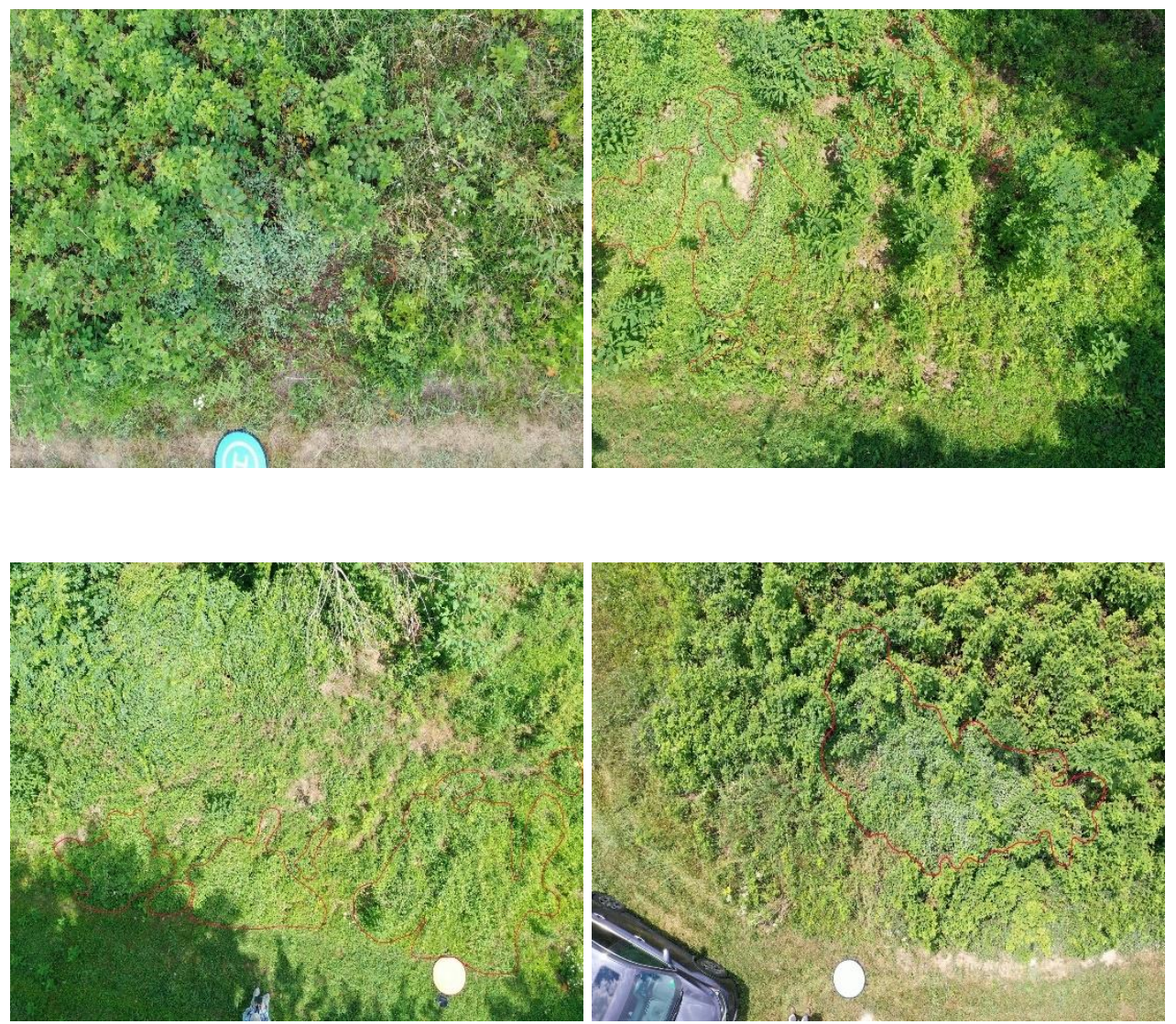

Figure 4.6: Real-time MAM aerial images with manually marked MAM regions.

In this thesis, we have used these MAM images to serve as our style images, and their style is imposed on the weed cluster images of the Agriculture Vision Dataset.

\subsubsection{Experiment setup:}

This section will focus on the experiment setup for our localized style transfer data augmentation. The hyperparameters details are mentioned in section 4.2.4, and the VGG19 network is initialized by using the content image (in our case Agriculture-Vision Images). The 
original image size of the content image is $5472 \times 3648$. For this style transfer, we use average pooling instead of max pooling to get the average of each patch of the feature map. The loss function we have chosen is an L-BFGS optimizer for limiting memory usage. The learning rate is $1 \mathrm{e} 0$, and the number iterations are 500 and 1000 . We have 4 cases of results.

1. Content image colour preserving with 500 iterations.

2. Content image colour preserving with 1000 iterations.

3. Disregarding content image colour with 500 iterations.

4. Disregarding content image colour with 500 iterations.

The input content, mask, and style images are as follows:
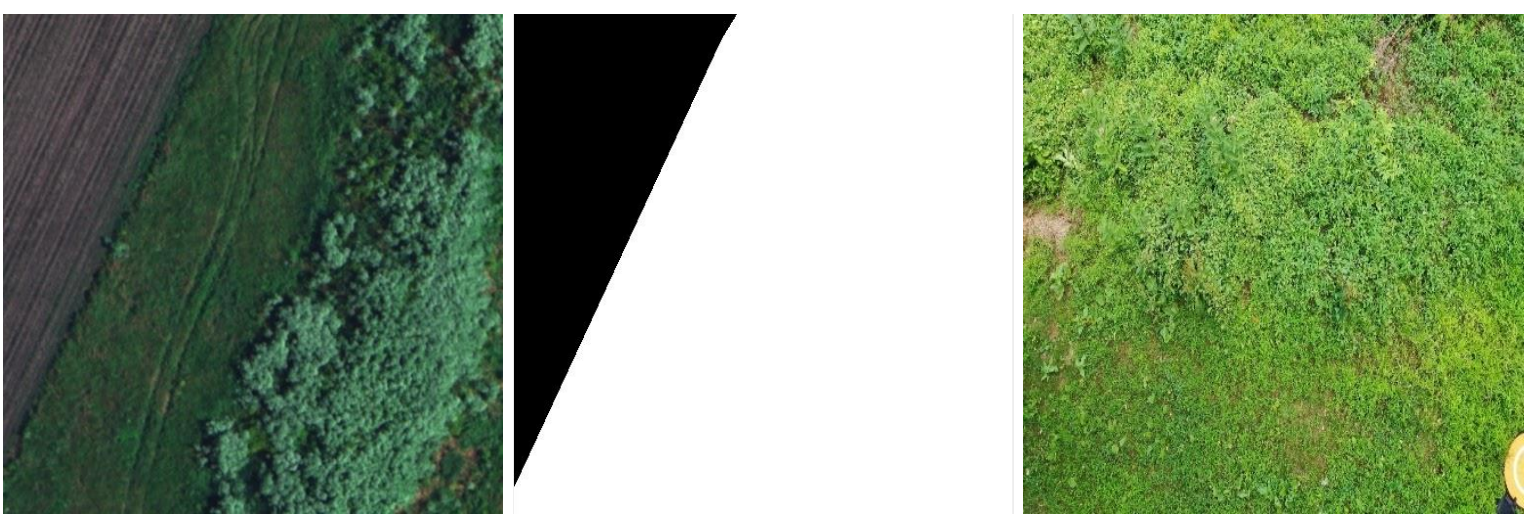

Figure 4.7: Content, Mask and Style images used for our data augmentation using NST.

The meaning of content colour preserving is that the original content image will retain its colour, and only the style from the style image is transferred to the content image but not the colour of the style image.

\subsubsection{Results and Discussion:}

The generated images in each of the above mentioned four cases are as follows: 


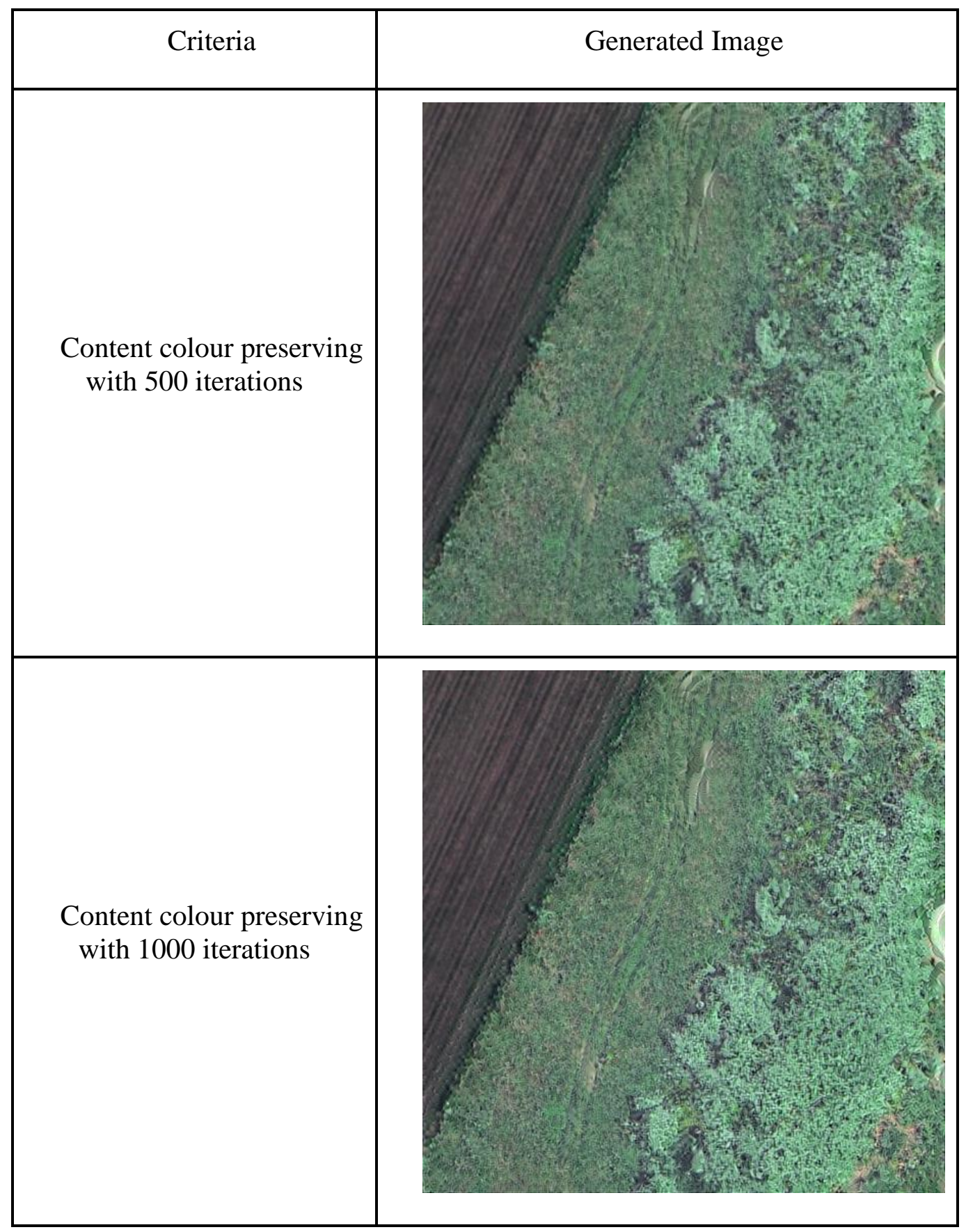




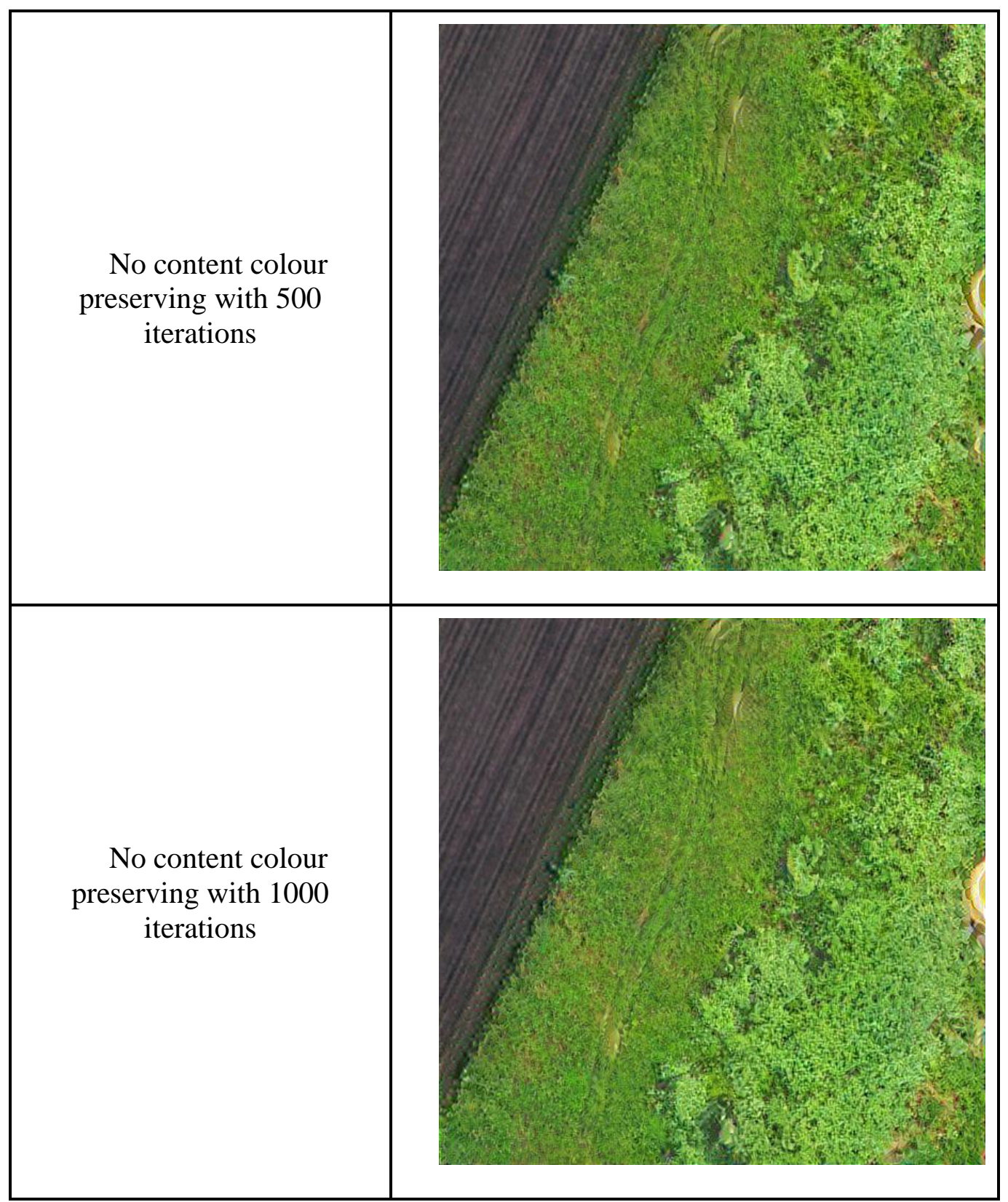

Table 5: Results of our NST data augmentation with VGG19

\section{Discussion:}

One of the pros of using NST for our data augmentation is that we can have various colour variations in our plant dataset and increase the number of images. The major con is that as we keep increasing the number of iterations, the images are looking more artistic than realistic, making it very hard for any model to understand the underlying patterns in the image. Without these pattern identifications, we cannot determine the species of the plants. The other con is that each image needs at least 1-2 minutes to go through the VGG 19. To produce a decent dataset, we need a 
minimum of 1500 images for training and 600 for validation. Using VGG19 for this will be computationally inefficient. As NST can't be used for recognition tasks, we moved on to another alternative to create our dataset.

\subsection{MAM Dataset with Geometrical Transformations:}

\subsubsection{Procedure:}

The second method of creating a dataset is by using geometrical transformations. In this thesis, we used Albumentations [48], an open-source library. The Albumentations library is often referred to as a CV tool that can improve Deep CNN's performance. A CNN's performance is improved because Albumentations' usage helps us create more data with the limited dataset. It is relatively fast and easy to use Albumentations to perform data augmentation. Albumentations can process around 70 different transformations on a single image. These transformations made it a powerful tool, and it is often used for object detection, instance detection tasks. As we are interested in object recognition, to be specific, detecting MAM regions from the MAM Aerial images shown in section 4.2.5, we have used a technique called Mask Augmentation. The procedure looks like follows:

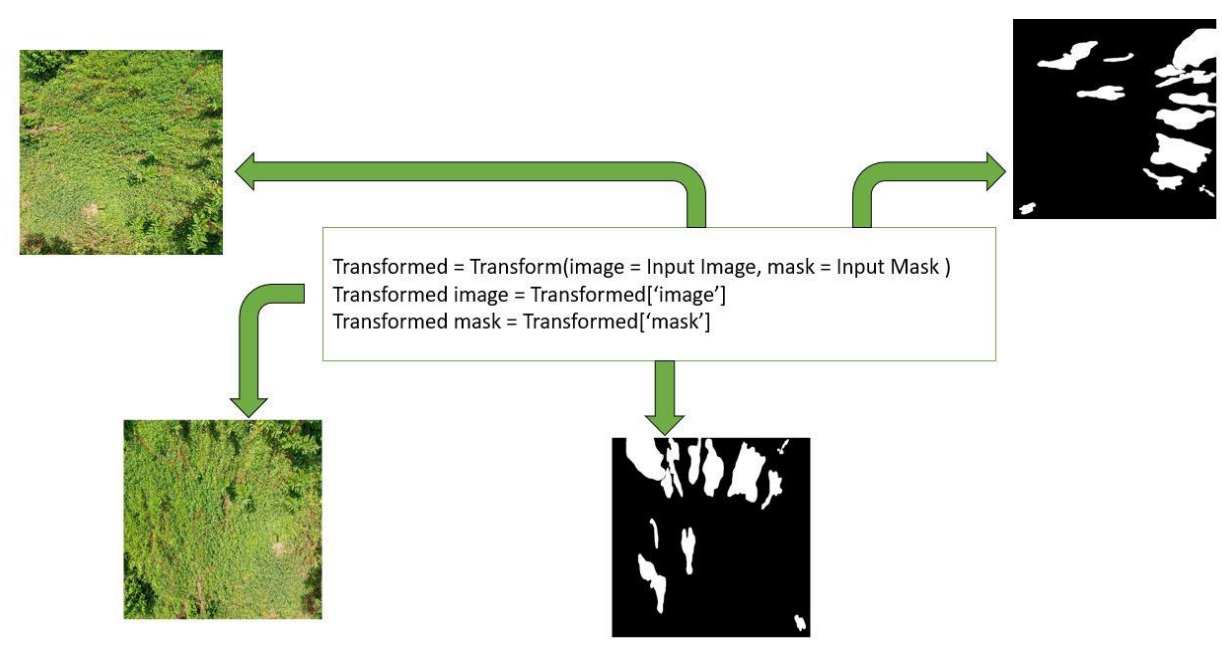

Figure 4.8: Procedure for mask augmentation using Albumentations.

We can use one or more masks for a single image. In the above example, each building is marked as a white zone or a pixel value 255 (normalized to 1), and the background is the black 
zone with a pixel value 0 . We have used the same technique to create our dataset as well. We marked all the regions with MAM as white zones and the rest as black. The figures below represent the RGB image and mask of our dataset.
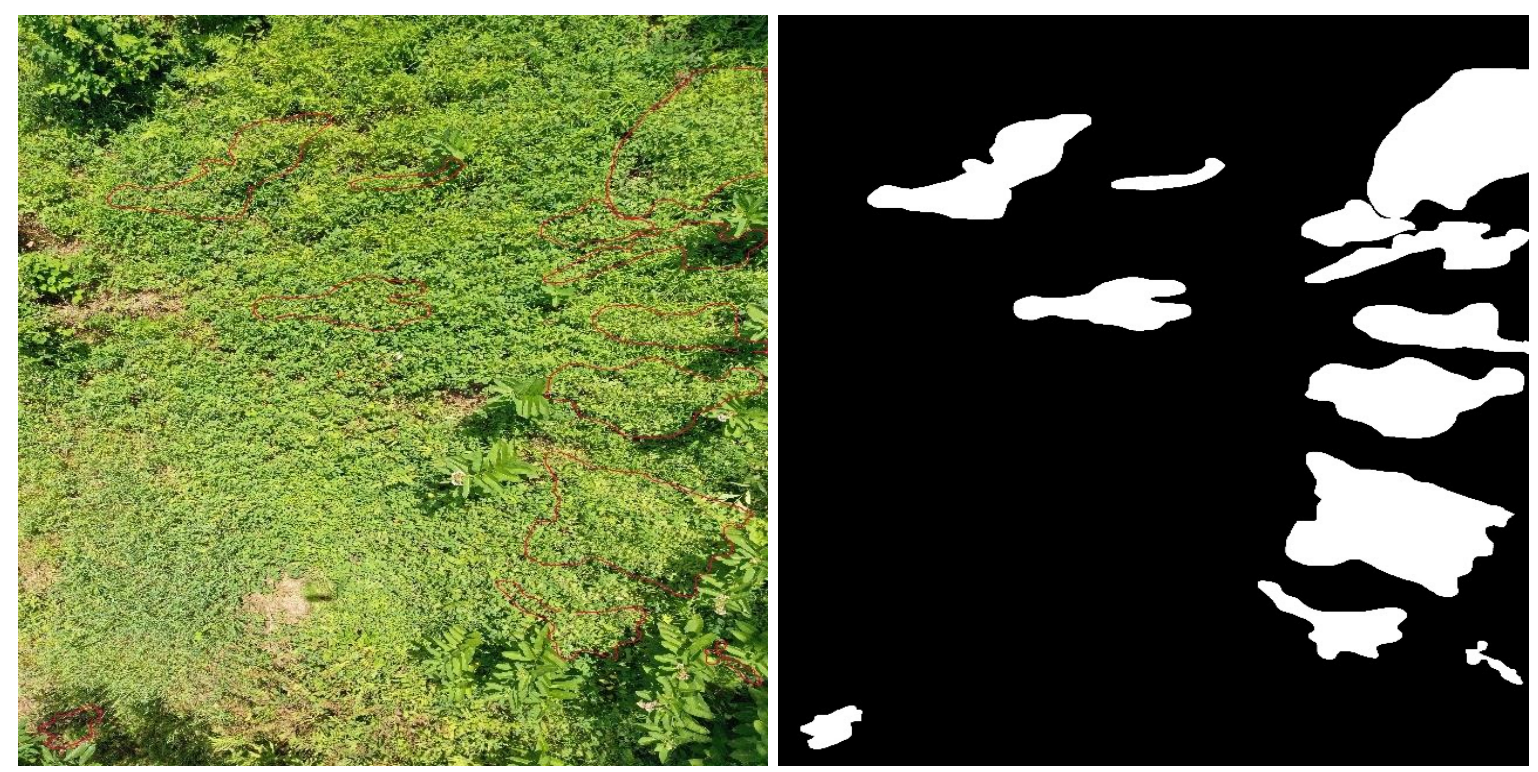

Figure 4.9: RGB (left) and binary mask (right) of the MAM aerial image.

The red lines in the RGB image are the boundaries of the MAM region in a field. Although Albumentations offer various transformations, we have used only 5 of them to create our fundamental dataset. The transformations applied on these RGB and binary mask image pairs are Centre crop, random 90 degrees rotation, grid distortion, and vertical and horizontal flips. Here are the images with the transformations. 


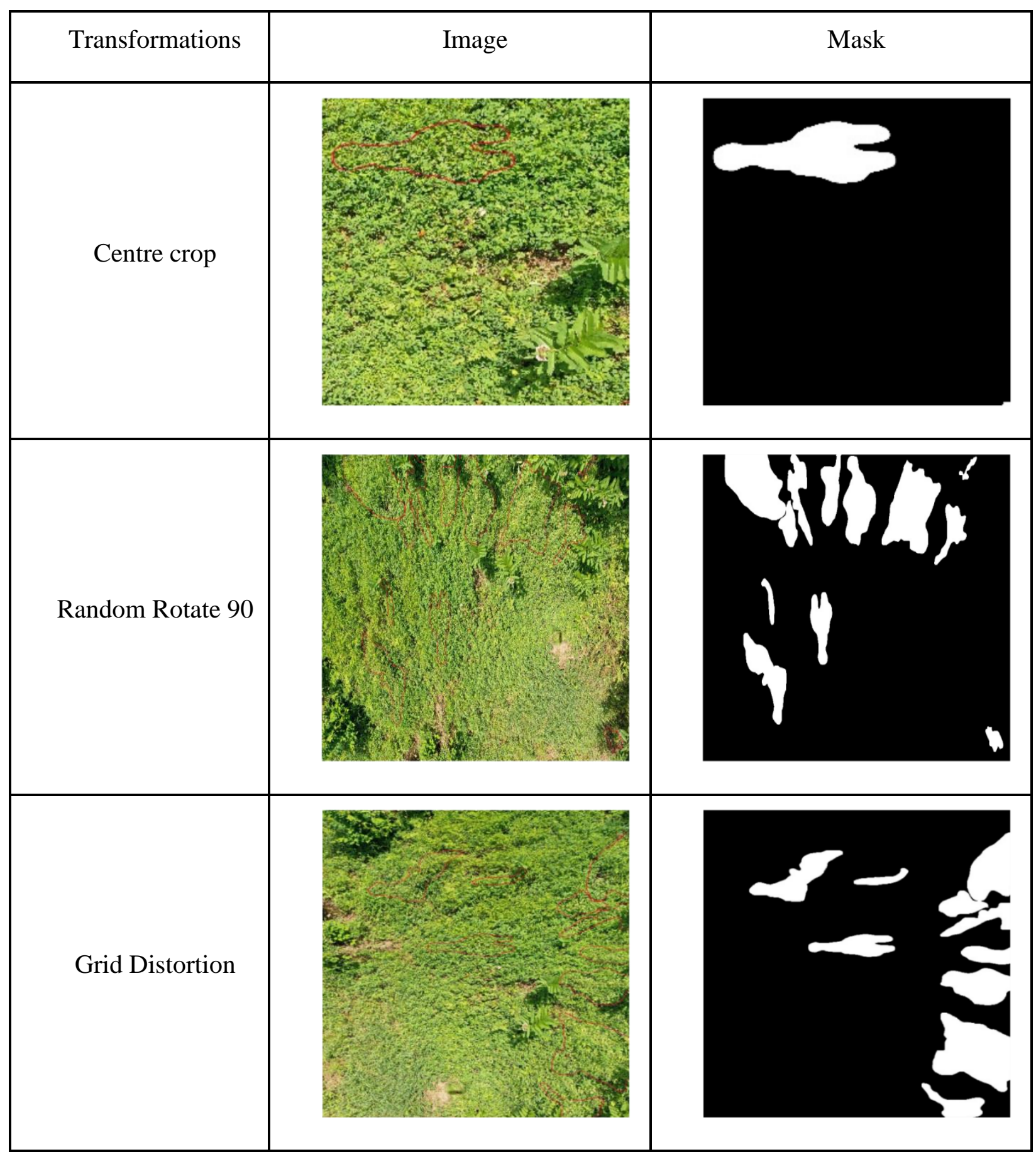




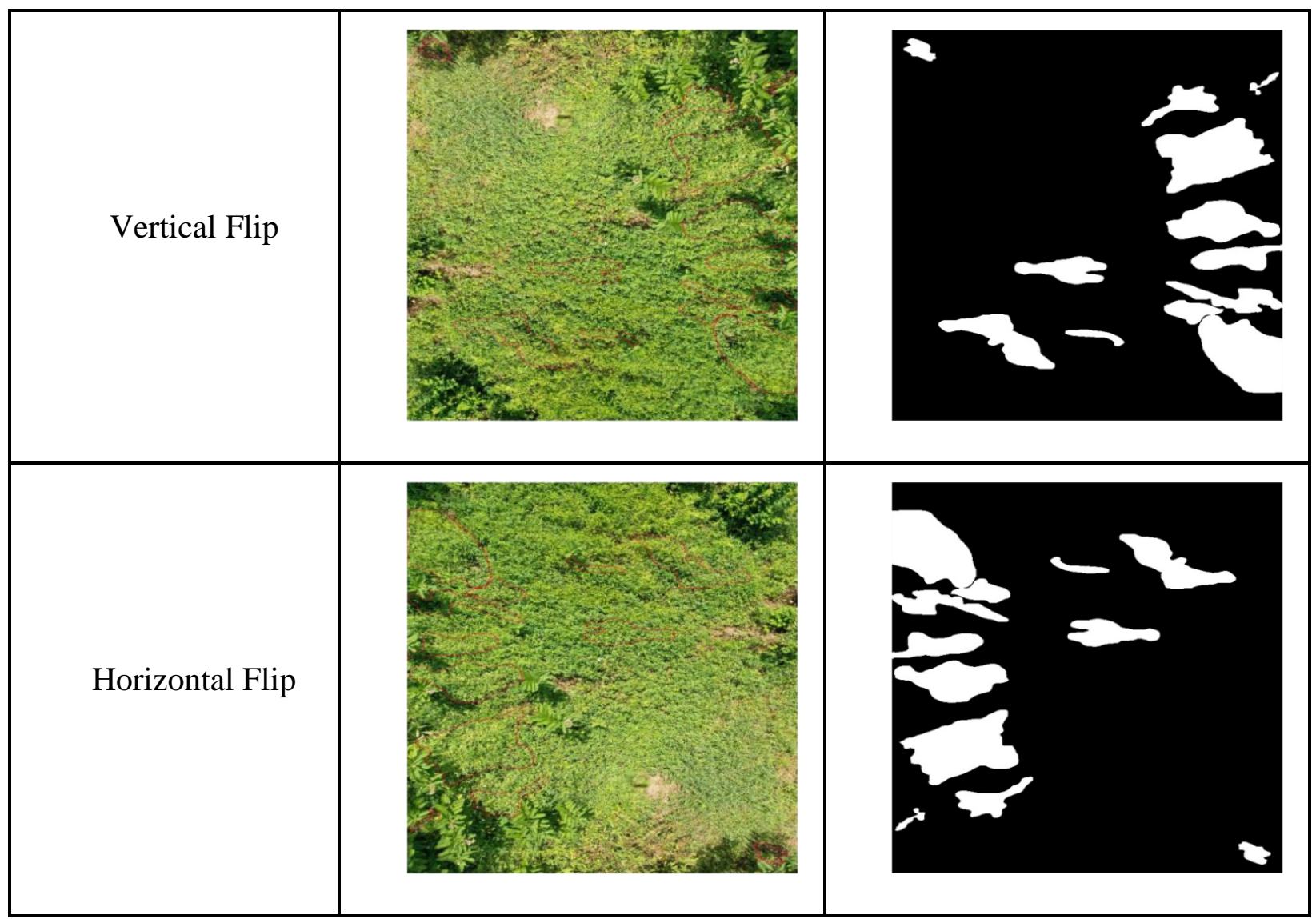

Table 6: Geometrical transformations on the RGB images and masks of aerial MAM data using Albumentations.

We applied the same transformations for the rest of the 14 images in a loop with three iterations and produced 2890 training images and 722 validation/testing images.

\subsubsection{Mask Generation:}

The binary mask seen in section 4.3.1 is produced by using mathematical operations and applying morphological filters [49] to the original RGB images. As the RGB images have a red boundary separating the MAM region and the rest, we used the red channel information of every RGB image and subtracted the red channel information from the grayscale image of the original RGB image. We then passed this difference image through a median filter [50] to remove the difference image's noise. A median filter is one of the non-linear digital filters for denoising and is often used in the post-processing phase. We have used MATLAB to achieve this task. The block diagram is shown below outlines the algorithm used: 


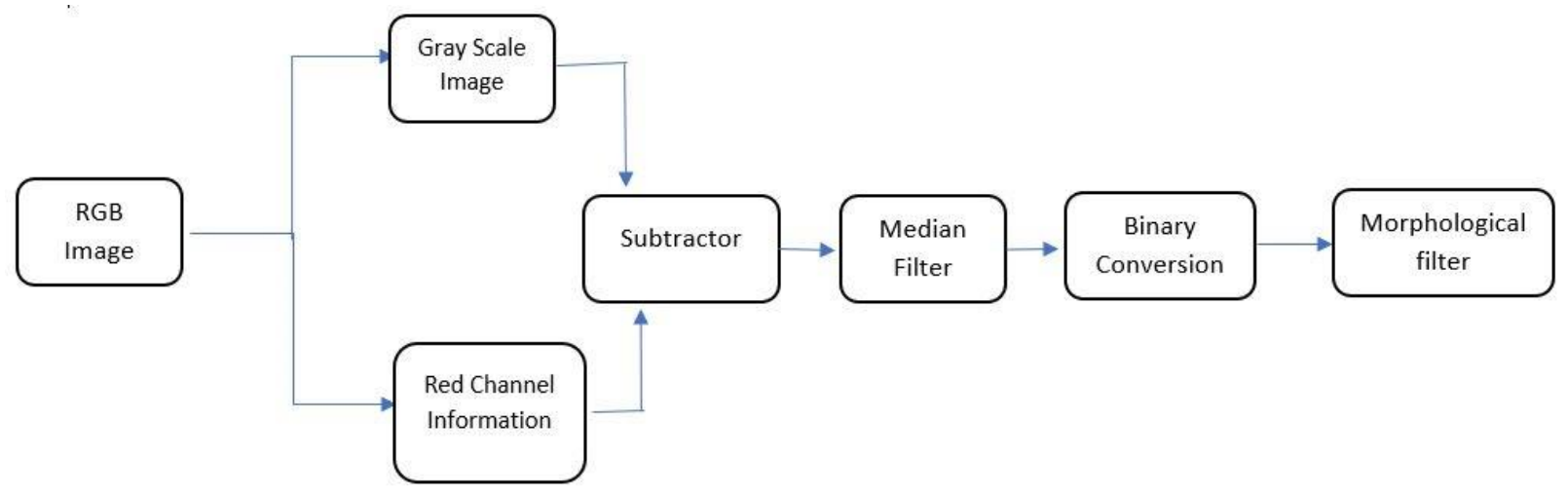

Figure 4.10: Block diagram for binary mask generation for the marked aerial RGB images.

Workflow:

1. The input image to the RGB image block to read the image.

2. Extract the red channel information and the grayscale information from the RGB image.

3. Find the difference between red channel and grayscale by using imsubtract.

4. To remove the noise, we applied a 2-D median filter with a 1x1 neighbourhood.

5. We transformed this denoised image into a binary image with a 0.5 level using im $2 \mathrm{bw}$.

6. Finally, we used a morphological reconstruction technique to fill the holes using the imfill function with 18 connectivities.

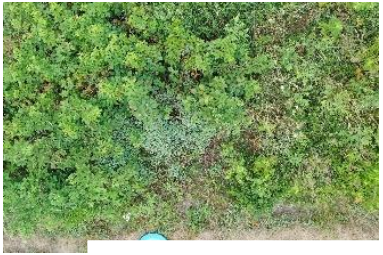

(1)

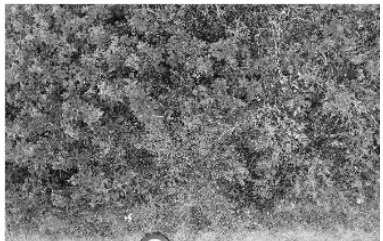

(2a)

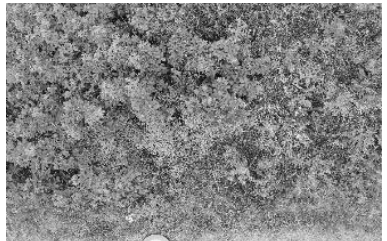

(2b)

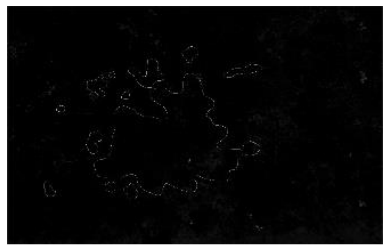

(3)

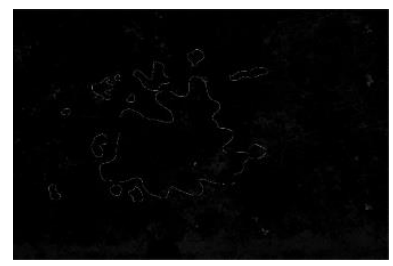

(4)

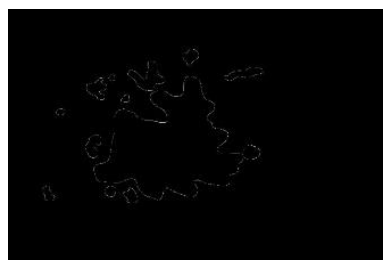

(5)

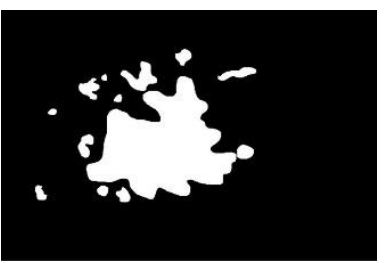

(6)

Figure 4.11: Output of each block. (1) RGB image, (2a) Red channel of the RGB image, (2b) Grayscale of the RGB image, (3) Difference of $2 a$ and $2 b$, (4) Difference image after denoising, (5) Binary image of the difference image, (6) Final mask after morphological reconstruction.

We iterated all the 15 images mentioned in section 4.3.1 to get the masks. 


\subsubsection{Annotations:}

As we are using the weights of Mask R-CNN trained on the COCO dataset, we need the annotations for our dataset to resemble the COCO dataset. As mentioned in section 3.4.2, we used the COCO standard format for annotation and saved it as a JSON file.

This dataset has only one super category called Weeds and only one object of interest, MAM. The bounding box values are obtained from the mask generated for each of the augmented images. With these annotations and the mask details, our dataset looks something like this:
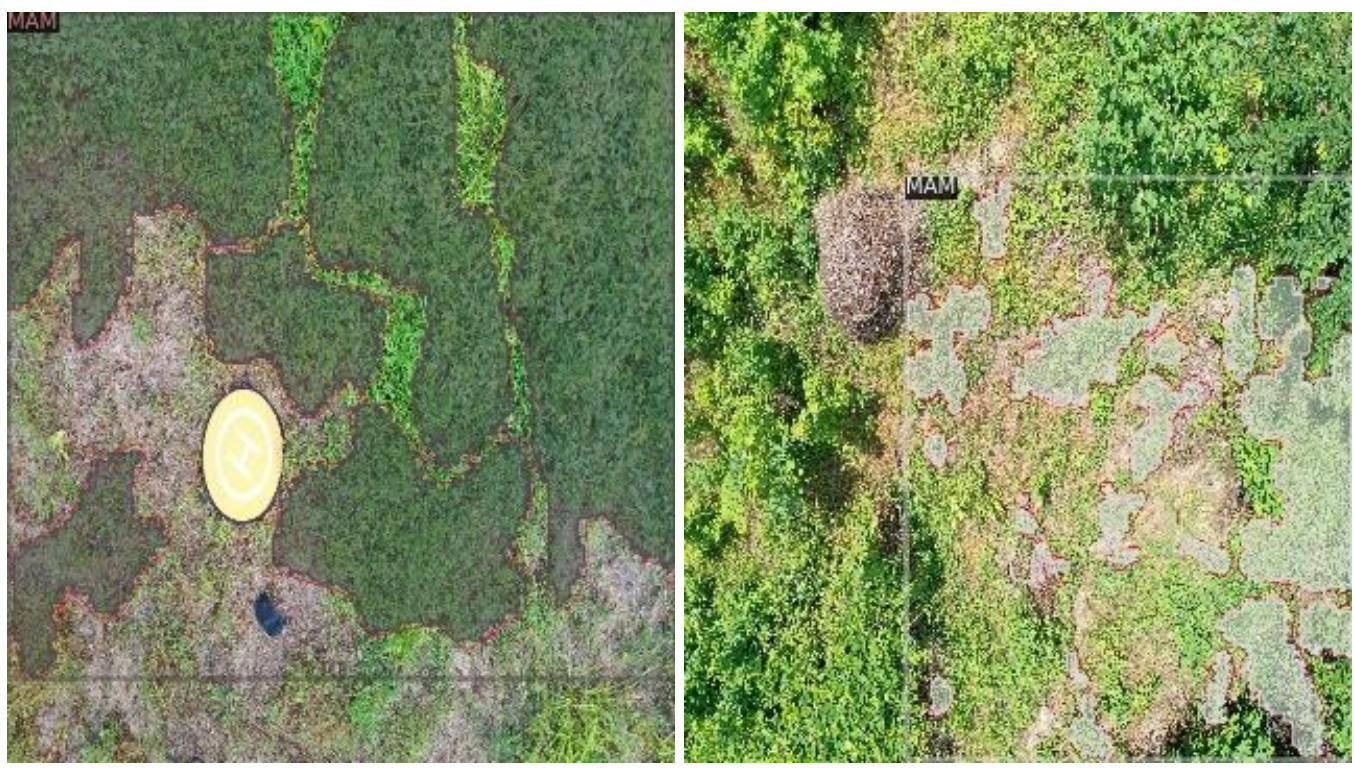

Figure 4.12: Example images from the Augmented MAM dataset.

\subsubsection{Limitations:}

Our dataset's limitations are that we have considered only 15 real-time drone images for our data augmentation. Any object detection model will not be able detect the MAM ideally in all weather and lighting conditions. Also, we have a red boundary around the MAM region in both training and testing images. The object detection models will most likely search for the red channel information to get to the MAM region. This drawback can be addressed by removing the humanmade red boundaries from the training and testing dataset. However, the only advantage of having these red boundaries is that we can better visualize the ground truth and the predicted values for the people who have no agricultural background. 


\section{Chapter 5:}

\section{MAM Detection using Mask R-CNN:}

\subsection{Introduction:}

The scientific name of MAM is Persicaria Perfoliata, formerly known as Polygonum Perfoliatum. The common names include devil's tail, Mile-A-Minute, and Asiatic tearthumb. It is a member of the polygonum family, often referred to as the buckwheat family. MAM falls under the Eukaryote domain's Plantae kingdom. This plant is native to India and East Asia. However, in the year 1930, it was accidentally introduced into the USA by contamination of holly seed. Now, MAM is found in almost all Mid-Atlantic states. MAM usually expands in cool areas because its seed needs a chilly period of eight weeks to flower. This plant smothers the other plants, trees, and shrubs by growing on top of them and has a growth rate of 6 inches per day. Because of this invasive nature, the plants underneath them will not receive enough sunlight for photosynthesis, and eventually, they become weak. The smothering is caused by the excessive weight and pressure caused by MAM. Although MAM in the US is only at $20 \%$ of its actual potential, it can decline the vegetation, thereby impacting the wildlife that survives on those plants. The reason for its potential to destroy the crop plants is that it is an invasive plant, meaning, in a foreign locale, the invasive plants will not have their natural enemies to stop their growth. The figures below show the occupancy of MAM in the US. 

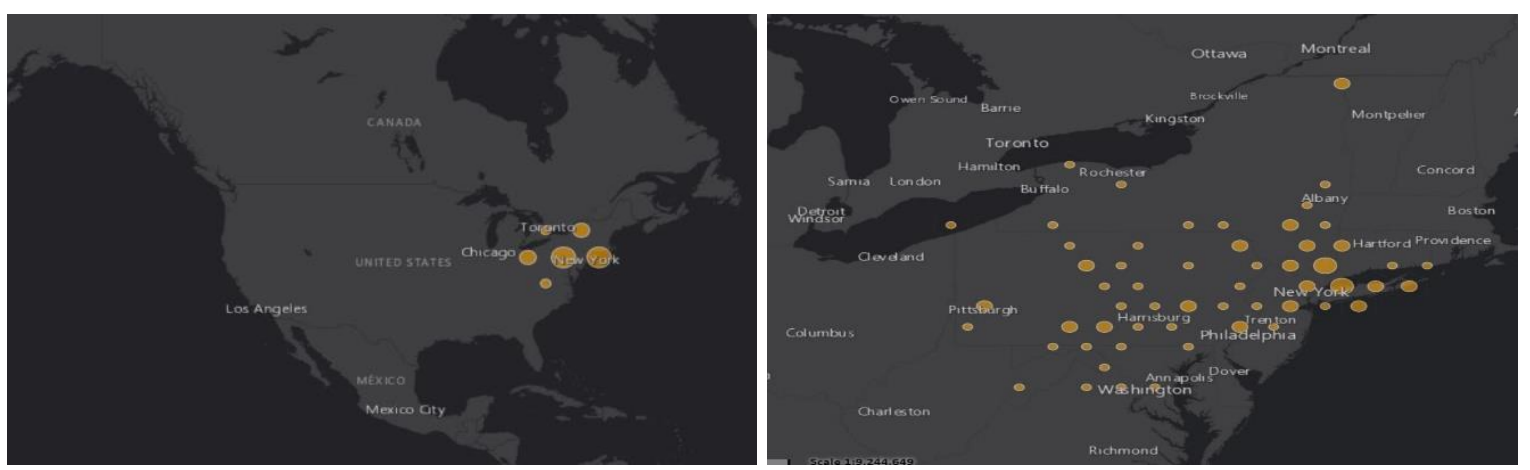

Figure 5.1: MAM infestation in the US (left) and areas in the east coast infected by MAM (right). Source (2 $^{32}$

\section{Description of MAM:}

MAM is an annual vine with light green leaves. The leaves' dimensions are usually $4-7 \mathrm{~cm}$ in height and 5-9 $\mathrm{cm}$ wide. The shape of the leaves is like an equilateral triangle. The vines change their shades from green to reddish shade, and as it is a vine, it has ocrea that surround the stems. The flowers are usually white, and the fruits resemble blue berry. MAMs fall under self-fertile plants as they do not need any pollinators for fertilization. June to October is the prime time in a year for their fertilization. The seeds that fall on the ground germinate around April through July. The spread of MAM can be due to these seeds as some birds carry these seeds to long distances. The birds, animals like squirrels, deer also eat MAM fruits, and their scat may contain MAM seeds. MAM seeds can float on water for nine days. So, any watershed, storms, or even vines hanging over water bodies can speed up the spreading of MAM. One more interesting fact about the seeds of MAM is that they can be viable in the soil for around six years, and they can start germination anytime. The figures below provide an idea of MAM.

\footnotetext{
32 http://nyis.info/invasive_species/mile-a-minute/.
} 

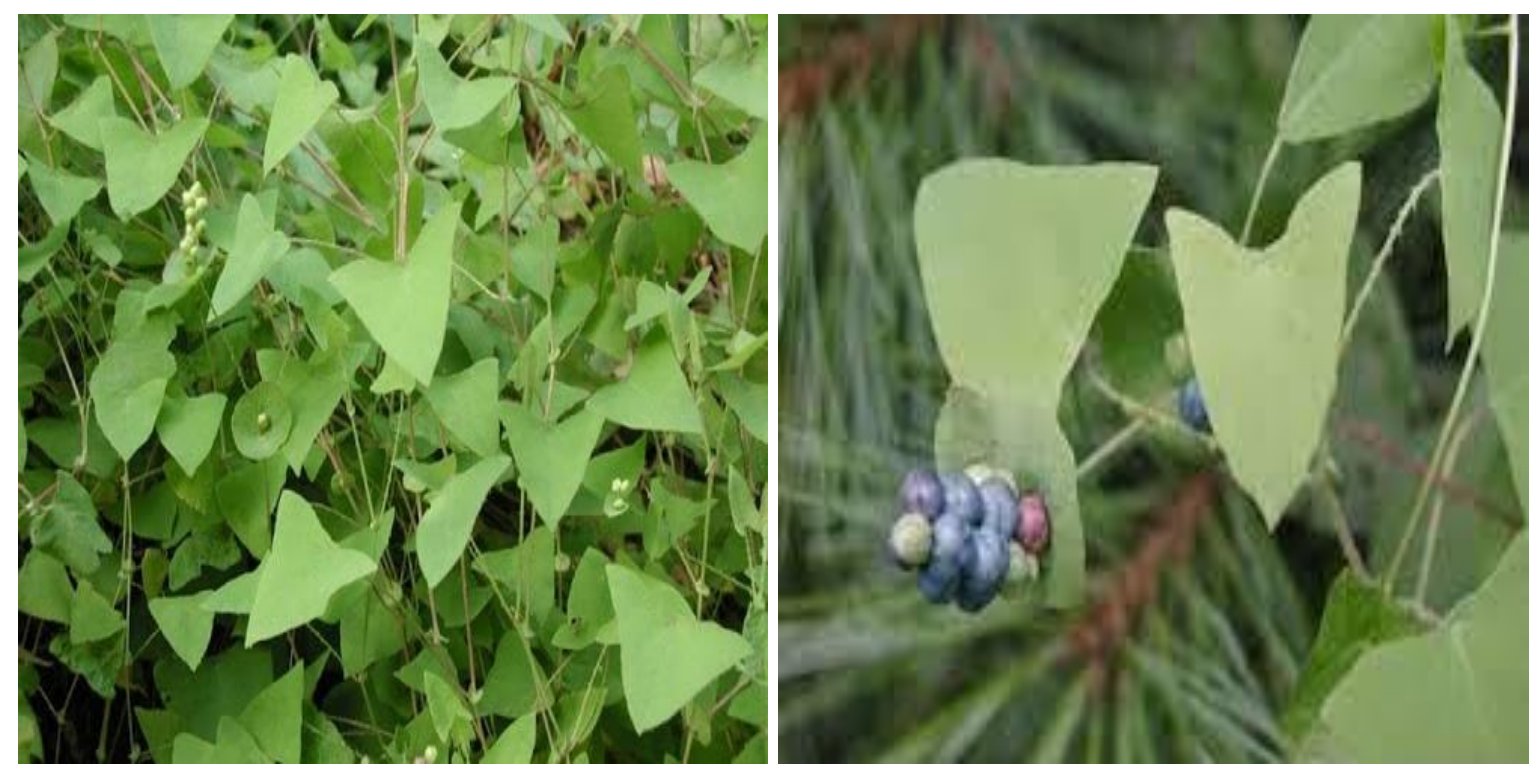

Figure 5.2: Leaves (left) and fruits (right) of MAM. Source ${ }^{33}$

\section{The habitat preferred by MAM:}

MAM plants are usually found in wet areas, but it is not the only criteria. It can also be seen in uncultivated farms, roadsides, water stream areas. Although MAM prefers moist soil, it can grow in dry soil as well. MAM also needs full sunlight. It grows over the top of other plants to reach for the sunlight. However, it can also grow under partial sunlight.

\section{MAM Management techniques:}

Once detected, we need to find a way to eradicate their presence. There are multiple techniques to do this job. Some of them are:

- Herbicides: Herbicides can be sprayed before the seed germinates or to a growing plant. The former type is called pre-emergent, and the latter type is called post-emergent. However, it can be challenging to spray herbicides because the MAM usually grows on top of a plant, and it is hard to localize the spraying on MAM.

\footnotetext{
${ }^{33}$ http://nyis.info/invasive_species/mile-a-minute/.
} 


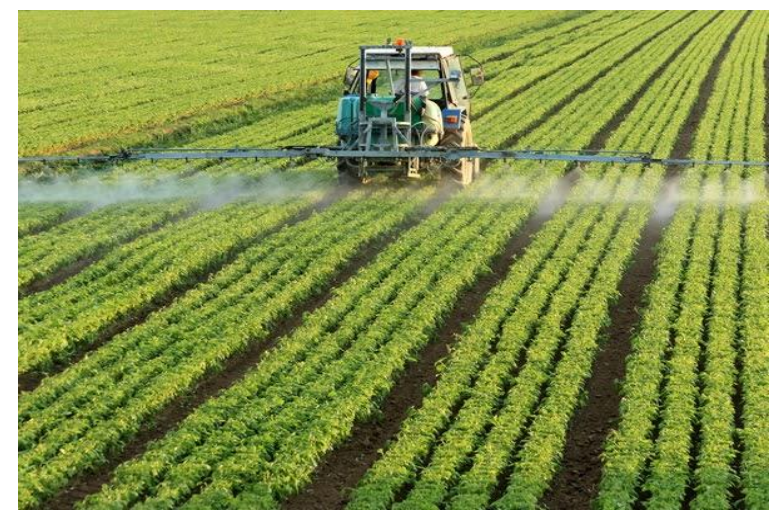

Figure 5.3: Spraying of herbicides for the entire field. Source ${ }^{34}$

- Manual labour: Hand plucking weeds is a tedious job but a very effective way to eradicate MAM with caution. We need to take extra care while plucking the plants with fruits as they may eventually spread more. Usage of gloves will be necessary if the barbs/thorns thicken. Once collected, they must be destroyed either by incineration or left dry.

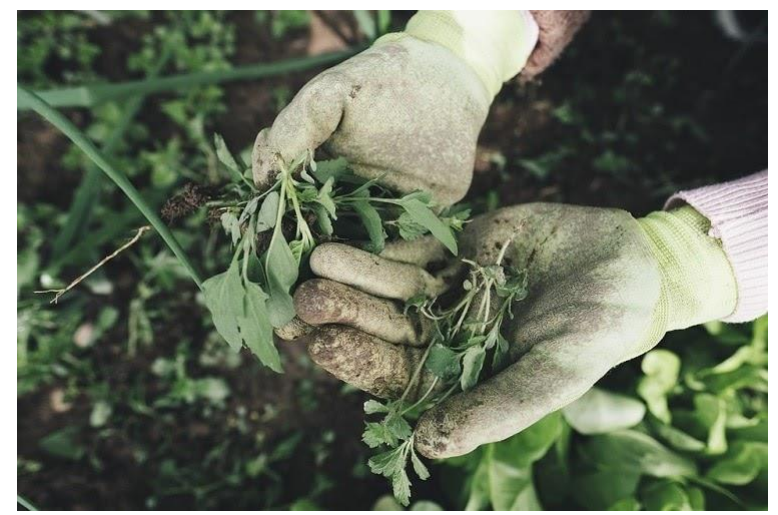

Figure 5.4: Hand plucking weeds. Source $e^{35}$

- Biological control: An insect that falls under weevils with the name Rhinocomimus Latipes Korotyaev popularly known as MAM weevil, can eradicate MAM. These insects are about $2 \mathrm{~mm}$ in length and have an orange film on top of their black body. MAM causes this film, hence the name MAM weevils. The adult insects feed on MAM, and the female ones lay eggs on the leaves. After pupation, the insects will start feeding on MAM again. Biological control is by far the effective and cheapest method for MAM eradication.

\footnotetext{
${ }^{34}$ http://lifeofplant.blogspot.com/2011/03/herbicides.html.

${ }^{35}$ https://askromapadaswami.com/digest-00574-plucking-weeds-by-romapadaswami.
} 


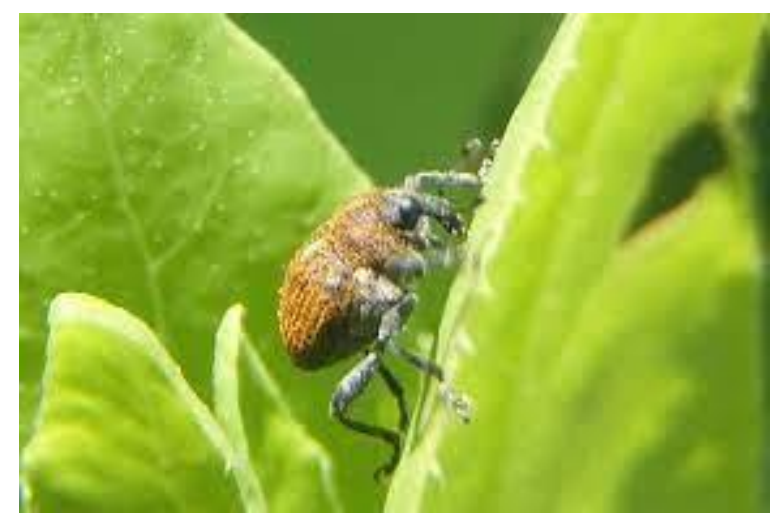

Figure 5.5: MAM weevil for biological control. Source ${ }^{36}$

Once the eradication is done, constant monitoring is required because MAM seeds can be viable for six years in the soil. Constant monitoring will help to reduce further spreading.

\section{Medicinal Values:}

Although MAM is notorious for destroying the natural habitat, it is an edible species. The shoots and tender leaves can be used in cooking as a vegetable or salad. It can be consumed raw as well. The fruits resemble blue berries, but they taste sweet. Along with these uses, it is used for various remedies in herbal medicine in China. In Traditional Chinese Medicine (TCM), MAM is often referred to as gang Bangui (杜板归).

\subsection{Three - Level Hierarchy for MAM detection:}

To detect MAM using UAVs and avoid false positives, we propose a three-level hierarchy (forest, trees, and leaves).

Stage 1 - Forest-level survey: The UAVs will be flying at higher altitudes to check for the agriculture field zones.

Stage 2 - Tree-level survey: Once the agriculture lands were confirmed, the UAVs will drop to a relatively low altitude, such as $10-15 \mathrm{~m}$, as shown in our dataset in section 4.3. We will use Mask R-CNN to locate the regions with MAM setting the threshold to $85 \%$.

\footnotetext{
${ }^{36}$ http://nyis.info/invasive_species/mile-a-minute/.
} 
Stage 3 - Leaf -level survey: After MAM region confirmation, the UAVs will further drop to $5 \mathrm{~m}$ to confirm the presence of MAM.

The flow chart is as follows:
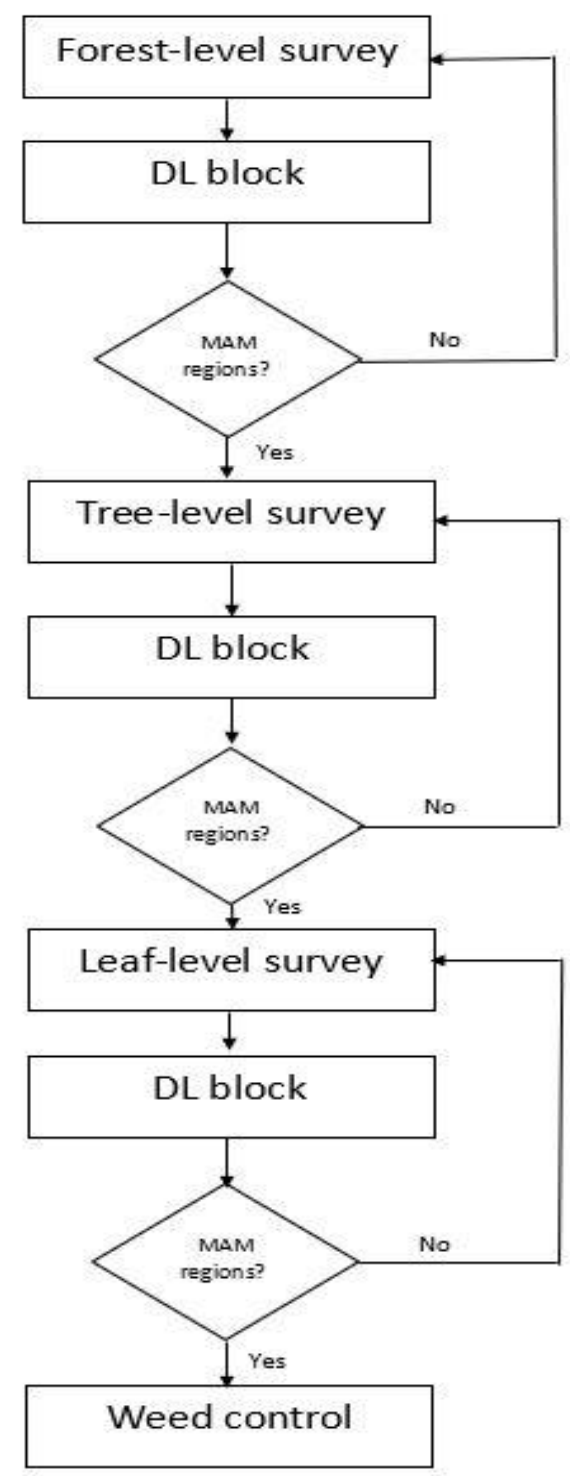

Figure 5.6: Flow chart for our three-level hierarchy

Here, the DL block is the Deep Learning block where we use Mask R-CNN for MAM region prediction. The weed control block can be biological control, herbicides, or manual plucking, as mentioned in section 5.1 


\subsection{Experiment Setup:}

In this section, we focused on the tree level analysis to detect the MAM presence using Mask R-CNN model. To train the model, we have used the dataset that we have created using the geometrical transformations in section 4.3. We have generated around 2890 images for training and 722 images for testing/validation. We have used Resnet 50 FPN as a backbone for feature extraction. The learning rate used is 0.001 , and the number of epochs is 300 . The batch size used is 16 , and the number of classes is two as we have MAM as one class and the other being the background. We used Detectron2 for this experiment because Detectron2 uses a PyTorch frame, as mentioned in chapter 2 , for faster and efficient performance.

\subsection{Results and Evaluations:}

For testing, we have set two thresholds $(0.9,0.5)$ for the Softmax. The following images will provide a detailed understanding of the results with the dataset created in section 4.3.
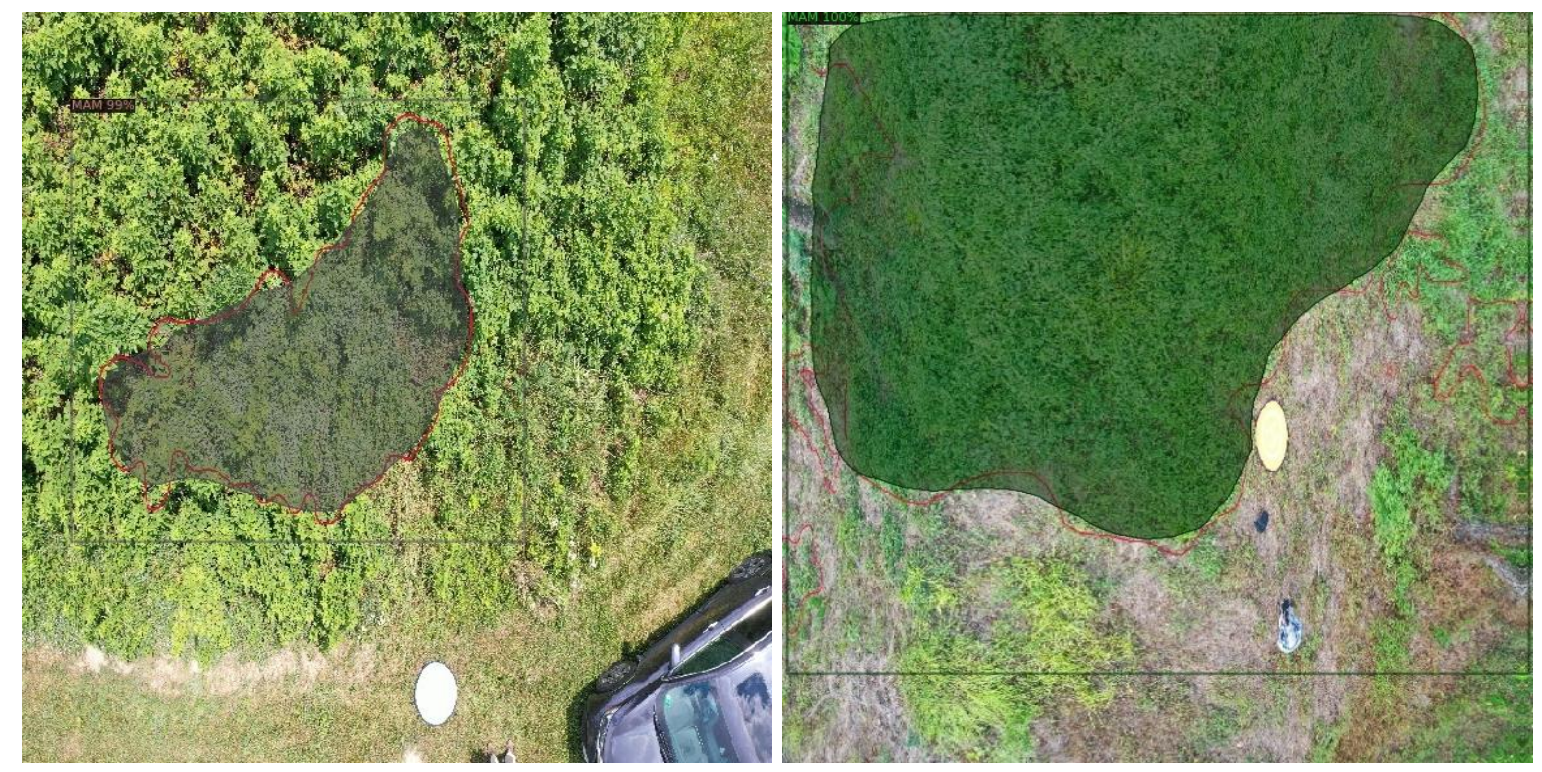

Figure 5.7: Augmented dataset's (section 4.3) Mask R-CNN results with Softmax threshold =0.9. The number on the top of the bounding box is the probability of MAM's presence.

In the above results, the red boundaries are our ground truth, and the mask is our prediction. The network does a pretty good job eliminating unwanted human-made objects such as the helipad, car, and the human himself. The number on the head of the RoI bounding box is the probability of 
that segmentation being MAM. Also, we can notice that all of the MAM regions were not detected on the image on the right because it is not $90 \%$ sure to be MAM.

Now, if we set the threshold to 0.5 , the network does an OK job identifying those small MAM patches, but it also detects the other human-made objects as MAM as well with the slightest probability. The figures below will explain the 0.5 threshold case.
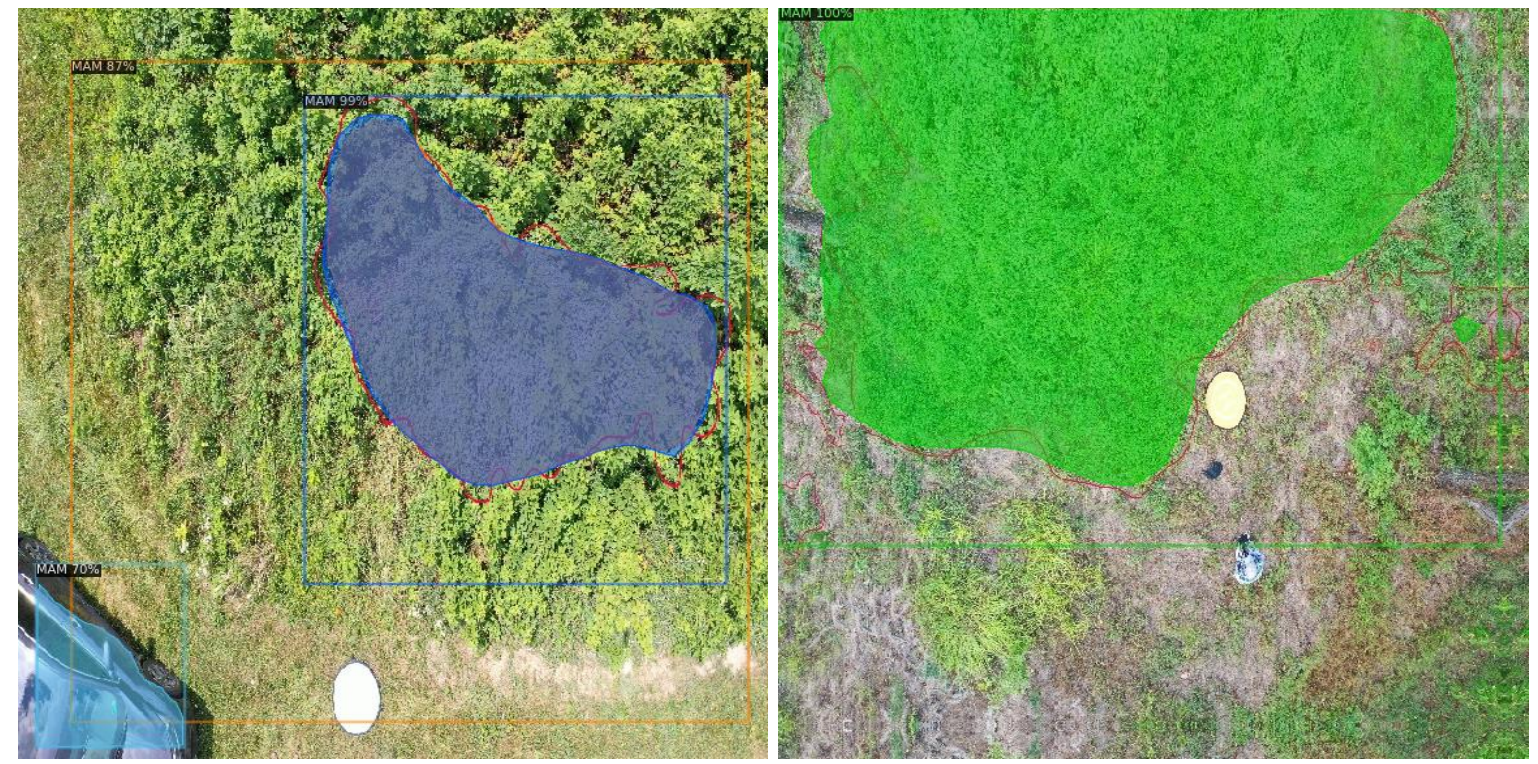

Figure 5.8: Augmented dataset's (section 4.3) Mask $R-C N N$ results with Softmax threshold $=0.5$. With this threshold, we have false alarms such as car detection.

As we did not specify anything about the car or helipad in our dataset, it is slightly tricky for the model to eliminate them because the dataset has a red line around the MAM region, and it is using the red channel information to learn the features of MAM. As the car and the other humanmade objects have shades closer to the MAM red line channel, it could be a miss-hit.

\section{Evaluation:}

As our dataset format resembles the COCO dataset and we have used the COCO pre-trained weights, we have used COCO evaluation metrics to understand our Mask R-CNN's overall performance. Our model has reached an Average Precision50 [32] of 98\% for the bounding box prediction and AP50 of $\sim 92 \%$. 


\subsection{Ablation study:}

As mentioned in section 3.3, we have used Feature Pyramid Network to serve as our Region Proposal Network (RPN) block for our Mask R-CNN model. This section will demonstrate why FPN is a better choice for RPN instead of others by comparing the Average Precision metrics of standard ResNet-50. We have used ResNet-50-C4 as our other backbone for RPN. Here, C4 represents the 4th stage of the convolutional final convolutional layer.

\begin{tabular}{|c|c|c|c|c|}
\hline Backbone & AP & AP50 & AP75 & APl \\
\hline ResNet-50-C4 & 61.772 & 87.274 & 77.221 & 61.772 \\
\hline ResNet-50-FPN & $\mathbf{7 0 . 0 1 9}$ & $\mathbf{9 8 . 8 5 5}$ & $\mathbf{8 4 . 8 9 5}$ & $\mathbf{7 0 . 0 1 9}$ \\
\hline
\end{tabular}

Table 7: COCO evaluation metrics for different RPN backbones.

The FPN backbone outperforms the standard ResNet-50-C4 when it comes to precision metrics. In ResNet-50-C4, we have a single-scale feature extractor at any given level in the RPN block. So, we need to use multiple anchors at every stage to extract the multi-scale information. These multiple anchor blocks slower the performance of our model. However, when this single-scale feature extractor is replaced with an FPN, we will have a single scale assigned to each layer, and at each level, multiple aspect ratios are used to extract the features. This pyramid architecture of FPN provides better accuracy and speed compared to the traditional ResNet backbones.

\section{Real-time testing:}

Although we have red ground truths in our training dataset, we have tested our model's performance on an unmarked original image. The results are as follows: 


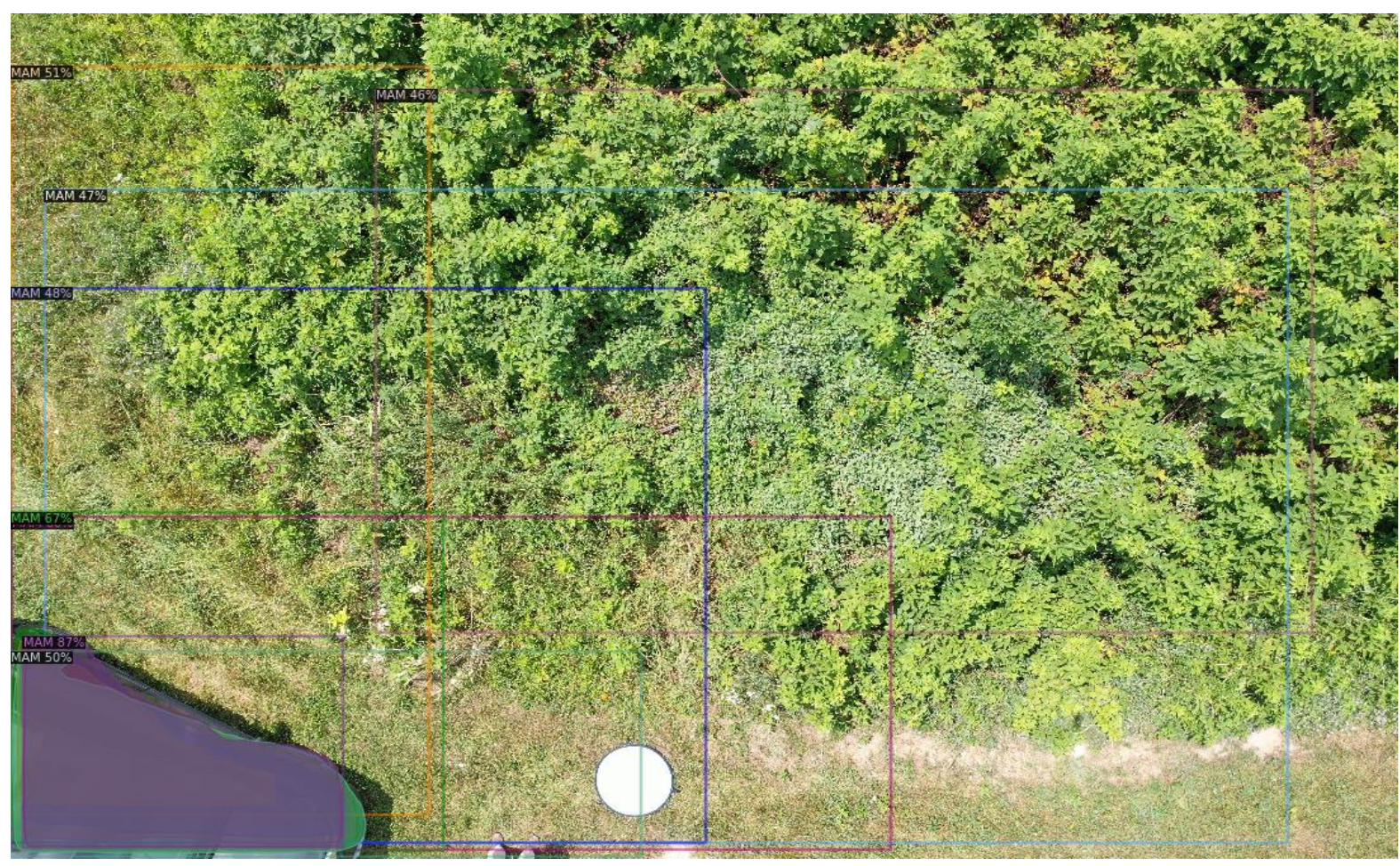

Figure 5.9: Mask R-CNN's performance on real-time data.

\section{Discussion:}

The model can still get the MAM region despite having a red boundary (46\% bounding box), but we have a lot of false alarms. To overcome this problem, we would have to remove the red markings around the RoI in our training image dataset. 


\section{Chapter 6:}

\section{Conclusions and Future Work:}

\subsection{Conclusions:}

In this thesis, we focused on recognition of weed plants in agricultural areas. We have tested Mask R-CNN's performance for recognizing plants with two classes and then expanded our experiment by creating a synthetic data of 80 classes for our field image study. For our aerial image study, we focused on various methods to create the dataset including localized style transfer and geometrical transformations. We have tested the performance of Mask R-CNN using this synthetic aerial image dataset and were able to reach an AP50 of 91\% for the predicted segmentation mask and $\sim 95 \%$ for the predicted bounding box.

\subsection{Future Work:}

Although we have satisfying results, there are certain limitations to our dataset. We have considered only one specific lighting condition and background plants as all the images we have generated using data augmentation technique are from 15 real time images. It can be quite challenging to recognize the plant species instead of just detecting one specific species. The following are the proposals for each stage in our hierarchy. 


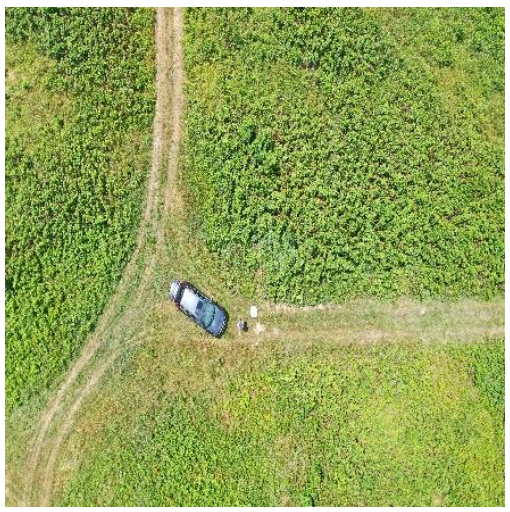

(1)

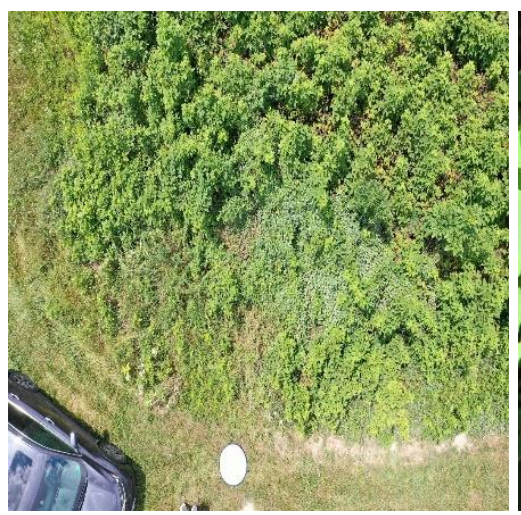

(2)

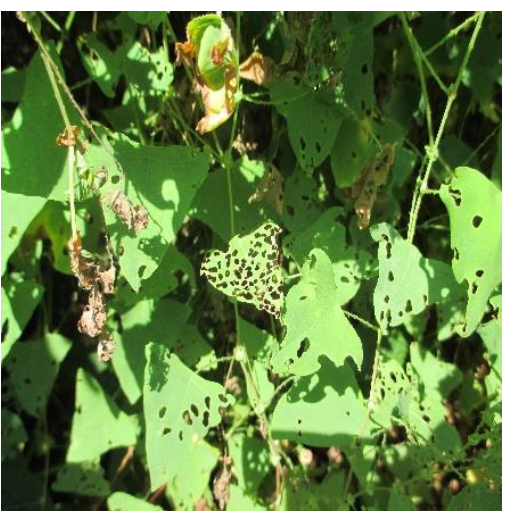

(3)

Figure 6.1: Three-level hierarchy. (1) Forest level, (2) Trees level, (3) Plant level.

\section{Proposals:}

Stage 1 - Forest-level survey: We need to come up with a good and properly annotated aerial image dataset to recognize the patterns of agriculture fields that contains MAM.

Stage 2 - Tree-level survey: Although we have a functional Mask R-CNN with decent AP, we must expand our dataset by considering images with various backgrounds like the plants that look similar to MAM and also consider various lighting and seasonal conditions.
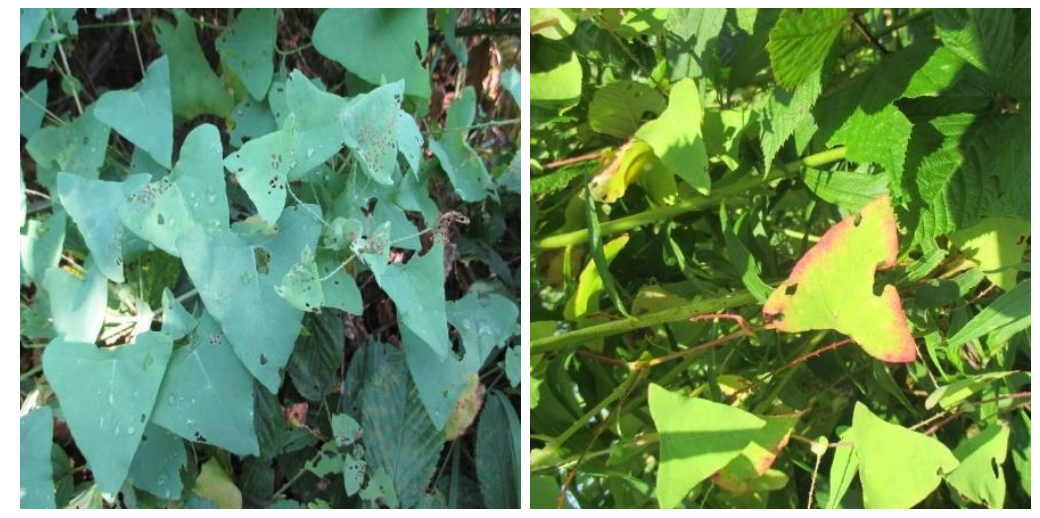

Figure 6.2: MAM in various lighting conditions.

Stage 3 - Leaf-level survey: We need to train a classifier that can classify MAM versus all the other common crop and weed plants that resembles MAM. For instance, a vine type called Microstegium Vimineum or commonly known as Japanese Stilt grass might be similar to 
MAM. But, the challenge of lack of proper dataset persists. We need to collect some images and apply Super Resolution (SR $)^{37}$ Techniques to improve the quality of the images collected from various sources and use those SR images to extract fine features.
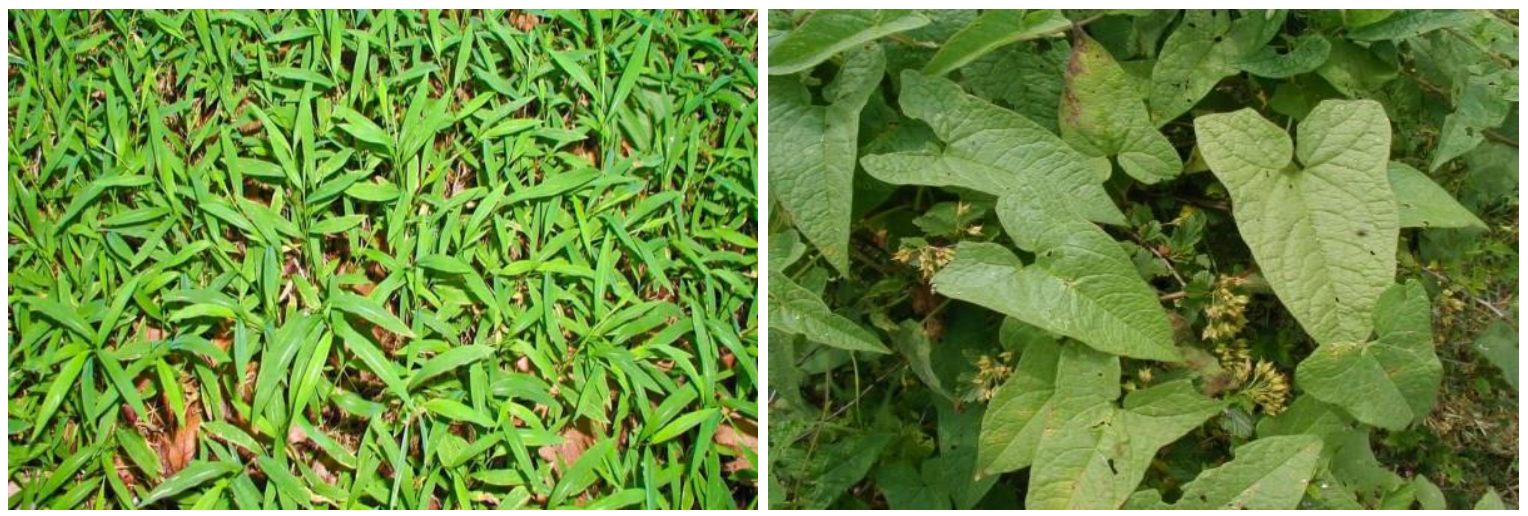

Figure 6.3: Weeds similar to MAM: Japanese Stilt grass (left) and Hedge Bindweed (right)

\footnotetext{
${ }^{37}$ A set of techniques to improve the resolution of an image.
} 


\section{Bibliography}

[1] Abouziena, H. F., and W. M. Haggag., "Weed control in clean agriculture: a review1.," Planta daninha 34.2, pp. 377-392, 2016.

[2] LeCun, Y., Bottou, L., Bengio, Y., \& Haffner, P., "Gradient-based learning applied to document recognition.," Proceedings of the IEEE, pp. 86(11), 2278-2324, 1998.

[3] Gatys, Leon A., Alexander S. Ecker, and Matthias Bethge., "A neural algorithm of artistic style.," arXiv preprint, p. arXiv:1508.06576, 2015.

[4] He, K., Gkioxari, G., Dollár, P., \& Girshick, R., “Mask r-cnn,” Proceedings of the IEEE international conference on computer vision, pp. 2961-2969, 2017.

[5] Haug, Sebastian, and Jörn Ostermann., "A crop/weed field image dataset for the evaluation of computer vision based precision agriculture tasks.," European Conference on Computer Vision. Springer, pp. 105-116, 2014.

[6] Chiu, M. T., Xu, X., Wei, Y., Huang, Z., Schwing, A. G., Brunner, R., ... \& Shi, H., "Agriculture-vision: A large aerial image database for agricultural pattern analysis.," In Proceedings of the IEEE/CVF Conference on Computer Vision and Pattern Recognition, pp. 2828-2838, 2020.

[7] Simonyan, Karen, and Andrew Zisserman., "Very deep convolutional networks for large-scale image recognition.," arXiv preprint arXiv:1409.1556, 2014.

[8] Mallah, C., Cope, J., \& Orwell, J. , "Plant Leaf Classification using Probabilistic Integration of Shape, Texture and Margin Features.," Pattern Recognit. Appl., 3842., 2013.

[9] Huang, Mei-Ling; Chang, Ya-Han, "Dataset of Tomato Leaves," Mendeley Data, V1, doi: 10.17632/ngdgg79rzb.1, 2020.

[10] CHOUHAN, Siddharth Singh; Kaul, Ajay; SINGH, UDAY PRATAP; \& Science, Madhav Institute of Technology , “ A Database of Leaf Images: Practice towards Plant 
Conservation with Plant Pathology,," Mendeley Data, V4, doi: 10.17632/hb74ynkjcn.4, 2020.

[11] Mohanty, S. P., Hughes, D. P., \& Salathé, M, “Using deep learning for image-based plant disease detection.," Frontiers in plant science, 7, 1419, 2016.

[12] Olsen, A., Konovalov, D. A., Philippa, B., Ridd, P., Wood, J. C., Johns, J., ... \& White, R. D. , "DeepWeeds: A multiclass weed species image dataset for deep learning.," Scientific reports, 9(1), 1-12., 2019.

[13] Lameski, Petre \& Zdravevski, Eftim \& Trajkovik, Vladimir \& Kulakov, Andrea. , "Weed Detection Dataset with RGB Images Taken Under Variable Light Conditions.," 112-119. 10.1007/978-3-319-67597-8_11. , 2017.

[14] Minervini, M., Fischbach, A., Scharr, H., \& Tsaftaris, S. A. , "Finely-grained annotated datasets for image-based plant phenotyping.," Pattern recognition letters, 81, 80-89., 2016.

[15] Giselsson, T., Dyrmann, M., Jlorgensen, R., Jensen, P., \& Midtiby, H., “ A Public Image Database for Benchmark of Plant Seedling Classification Algorithms.," arXiv preprint., 2017.

[16] Sudars, K., Jasko, J., Namatevs, I., Ozola, L., \& Badaukis, N. , "Dataset of annotated food crops and weed images for robotic computer vision control.," Data in brief, 31, 105833. https://doi.org/10.1016/j.dib.2020.105833, 2020.

[17] Wang, A., Zhang, W., \& Wei, X. , “A review on weed detection using ground-based machine vision and image processing techniques.," Computers and electronics in agriculture, Vols. 158, 226-240, 2019.

[18] Bakhshipour, A., \& Jafari, A. , “ Evaluation of support vector machine and artificial neural networks in weed detection using shape features," Computers and Electronics in Agriculture, Vols. 145, 153-160, 2018. 
[19] Yu, J., Sharpe, S. M., Schumann, A. W., \& Boyd, N. S. , "Deep learning for imagebased weed detection in turfgrass.," European journal of agronomy, Vols. 104, 78-84, 2019.

[20] Yu, J., Schumann, A. W., Cao, Z., Sharpe, S. M., \& Boyd, N. S. , “Weed detection in perennial ryegrass with deep learning convolutional neural network.," frontiers in Plant Science, no. 10, 1422, 2019.

[21] dos Santos Ferreira, A., Freitas, D. M., da Silva, G. G., Pistori, H., \& Folhes, M. T. , "Weed detection in soybean crops using ConvNets," Computers and Electronics in Agriculture, Vols. 143, 314-324, 2017.

[22] Louargant, M., Jones, G., Faroux, R., Paoli, J. N., Maillot, T., Gée, C., \& Villette, S. , "Unsupervised classification algorithm for early weed detection in row-crops by combining spatial and spectral information," Remote Sensing, no. 10(5), 761, 2018.

[23] Bah, M. D., Hafiane, A., \& Canals, R. , "Deep learning with unsupervised data labeling for weed detection in line crops in UAV images.," Remote sensing, 10(11), 1690., 2018.

[24] Hertzmann, A., Jacobs, C. E., Oliver, N., Curless, B., \& Salesin, D. H. , "Image analogies," In Proceedings of the 28th annual conference on Computer graphics and interactive techniques, pp. 327-340, 2001.

[25] Efros, Alexei A., and William T. Freeman., "Image quilting for texture synthesis and transfer.," Proceedings of the 28th annual conference on Computer graphics and interactive techniques., 2001.

[26] Jetchev, N., Bergmann, U., \& Vollgraf, R., "Texture Synthesis with Spatial Generative Adversarial Networks.," arXiv preprint arXiv:1611.08207, 2016.

[27] Bergmann, U., Jetchev, N., \& Vollgraf, R. , "Learning Texture Manifolds with the Periodic Spatial GAN.," arXiv preprint arXiv:1705.06566, 2017. 
[28] A. J. W. a. M. X. Wells, "Localized Style Transfer".

[29] Gatys, L. A., Bethge, M., Hertzmann, A., \& Shechtman, E, "Preserving color in neural artistic style transfer," arXiv preprint arXiv:1606.05897, 2016.

[30] Zheng, X., Chalasani, T., Ghosal, K., Lutz, S., \& Smolic, A. , "Stada: Style transfer as data augmentation.," arXiv preprint arXiv:1909.01056., 2019.

[31] Howard, Andrew G., Menglong Zhu, Bo Chen, Dmitry Kalenichenko, Weijun Wang, Tobias Weyand, Marco Andreetto, and Hartwig Adam., "Mobilenets: Efficient convolutional neural networks for mobile vision applications.," arXiv preprint arXiv:1704.04861, 2017.

[32] Lin, T. Y., Maire, M., Belongie, S., Hays, J., Perona, P., Ramanan, D., ... \& Zitnick, C. L., "Microsoft coco: Common objects in context.," European conference on computer vision. Springer, Cham, pp. 740-755, 2014.

[33] Ren, S., He, K., Girshick, R., \& Sun, J. , "Faster R-CNN: towards real-time object detection with region proposal networks.," IEEE transactions on pattern analysis and machine intelligence, 39(6), 1137-1149., 2016.

[34] Lin, T. Y., Goyal, P., Girshick, R., He, K., \& Dollár, P., "Focal loss for dense object detection.," In Proceedings of the IEEE international conference on computer vision, pp. 2980-2988, 2017.

[35] Cai, Z., \& Vasconcelos, N., "Cascade r-cnn: Delving into high quality object detection.," In Proceedings of the IEEE conference on computer vision and pattern recognition, pp. 6154-6162, 2018.

[36] Kirillov, A., Girshick, R., He, K., \& Dollár, P. , "Panoptic Feature Pyramid Networks," In Proceedings of the IEEE/CVF Conference on Computer Vision and Pattern Recognition , pp. 6399-6408, 2019. 
[37] S. Hochreiter, "The vanishing gradient problem during learning recurrent neural nets and problem solutions.," International Journal of Uncertainty, Fuzziness and Knowledge-Based Systems, 6(02), 107-116., 1998.

[38] Girshick, R., Donahue, J., Darrell, T., \& Malik, J. , "Rich feature hierarchies for accurate object detection and semantic segmentation.," In Proceedings of the IEEE conference on computer vision and pattern recognition, pp. 580-587, 2014.

[39] Felzenszwalb, P. F., \& Huttenlocher, D. P., "Efficient graph-based image segmentation," International journal of computer vision 59(2) , 167-181, 2004.

[40] R. Girshick, "Fast r-cnn," Proceedings of the IEEE international conference on computer vision., 2015.

[41] Ren, S., He, K., Girshick, R., \& Sun, J., "Faster r-cnn: Towards real-time object detection with region proposal networks.," arXiv preprint arXiv:1506.01497., 2015.

[42] Lin, T. Y., Dollár, P., Girshick, R., He, K., Hariharan, B., \& Belongie, S., "Feature Pyramid Networks for Object Detection," In Proceedings of the IEEE conference on computer vision and pattern recognition, pp. 2117-2125, 2017.

[43] He, K., Zhang, X., Ren, S., \& Sun, J. , “Deep Residual Learning for Image Recognition," In Proceedings of the IEEE conference on computer vision and pattern recognition, pp. 770-778, 2016.

[44] Roy, P., Ghosh, S., Bhattacharya, S., \& Pal, U., "Effects of Degradations on Deep Neural Network," arXiv preprint arXiv:1807.10108., 2018.

[45] Gatys, L. A., Ecker, A. S., \& Bethge, M., "Image style transfer using convolutional neural networks.," In Proceedings of the IEEE conference on computer vision and pattern recognition, pp. 2414-2423, 2016. 
[46] Deng, J., Dong, W., Socher, R., Li, L. J., Li, K., \& Fei-Fei, L., “Imagenet: A largescale hierarchical image database," In 2009 IEEE conference on computer vision and pattern recognition, pp. 248-255.

[47] Champandard, Alex J., "Semantic style transfer and turning two-bit doodles into fine artworks.," arXiv preprint arXiv:1603.01768, 2016.

[48] A. Buslaev, V., \& A. A. Kalinin, "Albumentations: fast and flexible image augmentations.," ArXiv e-prints, 2018.

[49] Serra, J., \& Vincent, L. , "An overview of morphological filtering.," Circuits, Systems and Signal Processing, 11(1), 47-108., 1992.

[50] Arce, G. R., Gallagher, N. C., \& Nodes, T. A., "Median filters: theory for one-and two-dimensional filters.," Advances in computer vision and image processing, 2, 89166., 1986.

[51] Zheng, Yufeng \& Yang, Clifford \& Merkulov, Aleksey. , "Breast cancer screening using convolutional neural network and follow-up digital mammography.," 4. 10.1117/12.2304564., 2018. 